\title{
Finite order automorphisms and a parametrization of nilpotent orbits in $p$-adic Lie algebras
}

\author{
by
}

Ricardo Portilla

A dissertation submitted in partial fulfillment of the requirements for the degree of Doctor of Philosophy

(Mathematics)

in The University of Michigan

2011

Doctoral Committee:

Professor Stephen M. DeBacker, Co-Chair

Professor Gopal Prasad Co-Chair

Professor Dmitriy Boyarchenko

Professor Michael E. Zieve

Professor James P. Tappenden 
(C) Ricardo Portilla 2011

All Rights Reserved 
For my sister 


\section{ACKNOWLEDGEMENTS}

First and foremost, I thank my advisor, Stephen DeBacker, for his support and extensive comments on the work presented in this thesis. Without his guidance, inspiration, and constant encouragement, this work would not have been possible. I thank Gopal Prasad for many helpful conversations and corrections. In addition, I am grateful to Gopal for his excellent instruction in courses on Lie groups and Lie algebras at the University of Michigan.

I am lucky to have been surrounded by the math graduate students at the University of Michigan, and I especially thank Michelle Lee, Harlan Kadish, Nina White, Qian Yin, and Eugene Eisenstein for contributing to my development, both mathematically and personally. 


\section{TABLE OF CONTENTS}

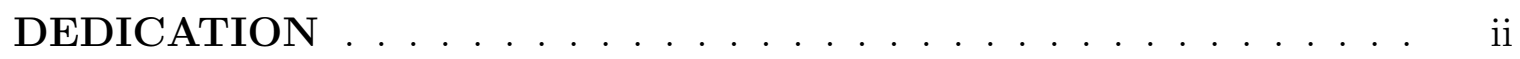

ACKNOWLEDGEMENTS ................... iii

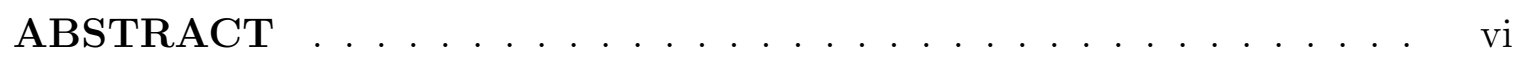

\section{CHAPTER}

I. Introduction $\ldots \ldots \ldots \ldots \ldots \ldots \ldots \ldots$

1.1 An overview of Bruhat-Tits theory . . . . . . . . . . . 1

1.2 Finite groups acting on reductive $p$-adic groups ....... 2

1.3 A parametrization of nilpotent orbits for $p$-adic symmetric spaces 3

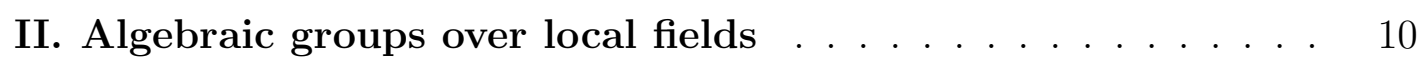

2.1 Algebraic groups, finite group actions, and associated notation 10

2.2 The Bruhat-Tits building, apartments, and $L$-fixed points . . 12

2.3 The Moy-Prasad filtrations . . . . . . . . . . . . . . . 13

2.3.1 A result of Prasad-Yu . . . . . . . . . . . . . . 14

III. Finite group actions on the Bruhat-Tits building $\ldots \ldots \ldots$

$3.1(r, L)$-facets and Moy-Prasad lattices . . . . . . . . . 16

3.2 Generalized $(r, L)$-facets . . . . . . . . . . . . . 22

3.3 Standard lifts and $r$-associativity $\ldots \ldots \ldots \ldots \ldots \ldots$

3.4 Identification of some $\mathfrak{f}$-vector spaces $\ldots \ldots \ldots \ldots$

3.5 An equivalence relation . . . . . . . . . . . . . 38

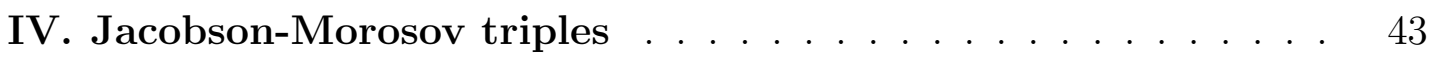

4.1 Some hypotheses . . . . . . . . . . . . . . . . . 45

4.1.1 Obtaining $\mathfrak{s l}_{2}(k)$-triples from $\mathfrak{s l}_{2}(\mathfrak{f})$-triples . . . . . 51

4.2 One-parameter subgroups . . . . . . . . . . . . 53 
V. The main result: a parametrization of nilpotent orbits in the Lie algebra of a reductive $p$-adic group . . . . . . . . 56

5.1 The building set . . . . . . . . . . . . . . 56

5.2 Noticed orbits . . . . . . . . . . . . . . . . 64

5.2 .1 Conjugacy of triples over $f \ldots \ldots$. . . . . . . 65

5.2 .2 An equivalent notion of a noticed pair . . . . . . . 66

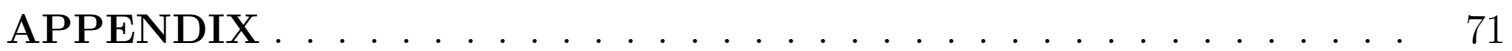

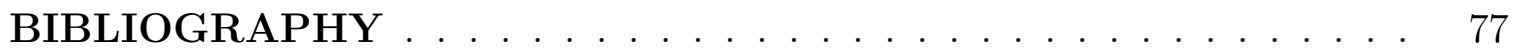




\begin{abstract}
Finite order automorphisms and a parametrization of nilpotent orbits in $p$-adic Lie algebras

by

Ricardo Portilla
\end{abstract}

Chair: Stephen DeBacker

Let $k$ be a field with a nontrivial discrete valuation which is complete and has perfect residue field. Let $G$ be the group of $k$-rational points of a reductive, linear algebraic group $\mathbf{G}$ equipped with a finite cyclic group $L$ acting on $\mathbf{G}$ by algebraic automorphisms defined over $k$. We assume that the Lie algebra of $G$ decomposes into a direct sum of eigenspaces, which we denote by $\mathfrak{g}^{i}$, under the action of $L$. If $\mathbf{H}$ is a $k$-subgroup of $\mathbf{G}^{L}$, the group of $L$-fixed points, which contains the neutral component of $\mathbf{G}^{L}$, then $H=\mathbf{H}(k)$ acts on each eigenspace of $\mathfrak{g}$, Let $r \in \mathbb{R}$. Under mild restrictions on the residual characteristic of $k$, the set of nilpotent $H$-orbits in the 1-eigenspace $\mathfrak{g}^{1}$ is parametrized by equivalence classes of noticed Moy-Prasad cosets of depth $r$ which lie in $\mathfrak{g}^{1}$. 


\section{CHAPTER I}

\section{Introduction}

\subsection{An overview of Bruhat-Tits theory}

In the 1970s, Bruhat and Tits (in [[4]] and [[5]]) made groundbreaking contributions to the structure theory of reductive $p$-adic groups $G$ by introducing the BruhatTits building, a closed, convex, contractible metric space with non-positive curvature on which $G$ acts by polysimplicial automorphisms. Their motivation came from the need to understand the structure of exceptional $p$-adic groups, and since then, the Bruhat-Tits building has been a powerful geometric tool used to study representations of a p-adic group. In 1994, Moy and Prasad (in [[21]]) used the Bruhat-Tits building of $G$, denoted $\mathcal{B}(G)$, to define filtrations of the Lie algebra of $G$ which were then used to define an invariant of a representation of a $p$-adic Lie group, referred to as the depth. More recent work of DeBacker ([[12]]), Yu (in [[35]]), and Hakim-Murnaghan ([[14]]) has highlighted its applicability to stability questions in representation theory as well as shedding light on distinguished supercuspidal representations and $p$-adic symmetric spaces.

The Bruhat-Tits building is a collection of (affine) Euclidean spaces called apartments which are glued together in such a way that encodes the structure of the $p$-adic group. Each apartment is partitioned into facets, which, in the case that $G$ is simple, are simplices. When $G$ is simple, the building of $G$ is a simplicial complex, 
the simplest nontrivial example being the group of $2 \times 2$ matrices of determinant one with entries in the 2 -adic numbers, denoted $G=S L_{2}\left(\mathbb{Q}_{2}\right)$. In this example, the Bruhat-Tits building of $S L_{2}\left(\mathbb{Q}_{2}\right)$ is a tree with valency three.

To each point $x$ in the Bruhat-Tits building, one can attach a group $G_{x}$, known as a parahoric subgroup of $G$. When $G$ is the group of $\mathbb{Q}_{p}$-rational points of a semisimple, simply-connected algebraic group, then the parahoric subgroup attached to a point $x$ is the stabilizer (in $G$ ) of $x$ under the action of $G$ on $\mathcal{B}(G)$. There is a normal subgroup $G_{x}^{+}$, known as the pro-unipotent radical of $G_{x}$, for which $G_{x} / G_{x}^{+}$is the group of rational points of a connected, reductive group over the residue field, which

we denote $\mathfrak{f}$. The work in this thesis examines nilpotent orbits in the Lie algebra of $G$ when there is an action of a finite group $L$ on $\mathbf{G}$ by automorphisms defined over a complete discretely valued field $k$. When $L$ acts on $\mathbf{G}$, there is a corresponding action on $\mathcal{B}(G)$ under which the set of parahoric subgroups and their pro-unipotent radicals are preserved. In this way, we have an $L$-action on the Lie algebra of $G_{x} / G_{x}^{+}$for any point $x \in \mathcal{B}(G)$ which is fixed by $L$. In this thesis, we adapt ideas in [[12]] to show that when $L$ is cyclic, nilpotent orbits (in the Lie algebra of $G$ ) under the action of the group of $L$-fixed points, denoted $G^{L}$, may be parametrized in terms of equivalence classes of Moy-Prasad cosets.

\subsection{Finite groups acting on reductive $p$-adic groups}

The work of Prasad-Yu ([[26], Theorem 1.9]) allows one to begin to understand the action of a finite group on a reductive $p$-adic group using the Bruhat-Tits building of $H:=G^{L}$, the group of rational points fixed under $L$. Namely, Prasad and Yu show that there is an identification

$$
\mathcal{B}(H)=\mathcal{B}(G)^{L},
$$


when the cardinality of $L$ is coprime to $p$. This work allows one to use Bruhat-Tits theory to study questions about the homogeneous space $G / H$, exploiting the fact that $H$ is a reductive $p$-adic group and using the Bruhat-Tits building of $H$. The work in this thesis is related to recent advances by Panyushev ([[23]]) and Levy ([[20]]), whose work is related to important results due to Popov ([[24]]). The work of Panyushev and Popov has recently been applied in the exciting work of Gross-Reeder-Yu ([13]) in which they establish a connection between Vinberg theory ([32]]) and Moy-Prasad theory.

\subsection{A parametrization of nilpotent orbits for $p$-adic symmet- ric spaces}

A motivation for the work in this thesis comes from the theory of $p$-adic symmetric spaces, in which the objective is to understand involutions acting on reductive groups; this is a special case for which $|L|=2$. More precisely, let $\theta: \mathbf{G} \rightarrow \mathbf{G}$ be a nontrivial involution defined over $k$. Under $d \theta$, the differential of $\theta$, the vector space of $k$-rational points of $\operatorname{Lie}(\mathbf{G})$, denoted $\mathfrak{g}$, decomposes into $(+1)$ and $(-1)$-eigenspaces, which we

denote $\mathfrak{h}$ and $\mathfrak{p}$, respectively. Let $\mathbf{H}$ denote a subgroup with $\left(\mathbf{G}^{\theta}\right)^{\circ} \subset \mathbf{H} \subset \mathbf{G}^{\theta}$ such that $\mathbf{H}$ is defined over $k$. Vust (in [[33]]) and Prasad-Yu (in [[26], Theorem 2.4]) showed that $\mathbf{H}^{\circ}$ is reductive whenever $\mathbf{G}$ is reductive. Thus, we may consider the Bruhat-Tits building of $H=\mathbf{H}(k)$, which we denote $\mathcal{B}(H)$. (Note that $H$ preserves $\mathfrak{p}$ under the adjoint action.) Under the assumption that the residual characteristic of $k$ is not two, Prasad and Yu showed (in [[26], Theorem 1.9]) that we may identify $\mathcal{B}(H)$ with the set of $\theta$-fixed points in the Bruhat-Tits building of $G$. This result was also proved in the case where $\mathbf{H}$ is a classical group arising from an involution (as well as spherical buildings) in [[17], Theorem 6.7.3]. Using this identification, it makes sense to think of elements of $\mathcal{B}(H)$ as elements lying in $\mathcal{B}(G)$. 
In [[21]], for each $r \in \mathbb{R}$, Moy and Prasad associate a lattice $\mathfrak{g}_{x, r}$ to each point $x \in \mathcal{B}(G)$. If $r=0$ and $x \in \mathcal{B}(H)$, then $\theta$ acts on each Lie algebra $V_{x, 0}:=\mathfrak{g}_{x, 0} / \mathfrak{g}_{x, 0^{+}}$, which then gives a decomposition $V_{x, 0}=V_{x, 0}^{+} \oplus V_{x, 0}^{-}$into $(+1)$ and (-1)-eigenspaces. We can then define an action of $H$ on the set of degenerate cosets in $V_{x, 0}^{-}$, meaning those cosets which contain a nilpotent element in $\mathfrak{g}$. Thus, in the case when $r=0$ and $|L|=2$, this thesis provides a parametrization of nilpotent $H$-orbits in $\mathfrak{p}$ in terms of equivalence classes of pairs $(F, e)$, where $F$ is the set of $\theta$-fixed points of a $\theta$-stable facet of $\mathcal{B}(G)$, and $e$ is a degenerate coset in $V_{x, 0}^{-}$.

It is hoped that the structure result presented in this thesis will be useful in harmonic analysis on $G / H$, which is referred to as a $p$-adic symmetric space. In the setting of $p$-adic symmetric spaces, spherical characters play the role of characters of irreducible, admissible representations of $G$. In [[27], Theorem 7.11], Rader and Rallis give a local expansion for spherical characters of irreducible class one representations of $G$ (see [[27], Section 1]) in a neighborhood about the identity in terms of $H$ invariant distributions supported on $\mathcal{N} \cap \mathfrak{p}$, the set of nilpotent elements in $\mathfrak{p}$. By the proof of [[28], Lemma 2.4], we can (and do) identify the $k$-rational points of the tangent space of $\mathbf{G} / \mathbf{H}$ at the identity with $\mathfrak{p}$, and this is where the nilpotent $H$-orbits will live. A motivation for describing a parametrization of nilpotent $H$-orbits in $\mathfrak{p}$ is to establish a homogeneity result about the spherical character of an irreducible class one representation of $G$. The analogous homogeneity result for characters of irreducible, admissible representations of $G$, which occurs in harmonic analysis on $G$, was given in [[9], Theorem 3.5.2].

In this thesis, we focus on a particular type of facet which encodes the $H$-orbit structure of $\mathcal{N}$. In particular, we suppose that $\mathfrak{g}$ is completely reducible as a representation of $L$ and fix an isotypic component $\mathfrak{g}^{\sigma}$, corresponding to some irreducible representation $\left(\sigma, V^{\sigma}\right)$ of $L$ contained in $\mathfrak{g}$. Suppose $r \in \mathbb{R}$. As in [[12], Section 3.1], we say that $x, y \in \mathcal{B}(G)$ belong to the same generalized r-facet $F^{\prime *} \subset \mathcal{B}(G)$ 
if $\mathfrak{g}_{x, r}=\mathfrak{g}_{y, r}$ and $\mathfrak{g}_{x, r^{+}}=\mathfrak{g}_{y, r^{+}}$. We will only consider the $L$-fixed points of $L$-stable generalized $r$-facets; we call these generalized $(r, L)$-facets. The generalized $(r, L)$ facets form a partition of the Bruhat-Tits building of $H$.

In Chapter III, we develop the theory of generalized $(r, L)$-facets for any finite group $L$, but in all subsequent chapters and for the main result, we restrict to the case where $L$ is a finite cyclic group. In this case, the Lie algebra of $G$ decomposes into isotypic components which are eigenspaces under the $L$-action. If $L$ is of order $m$, then the Lie algebra is the direct sum of eigenspaces $\mathfrak{g}^{i}$ where $i \in\{0,1, \ldots, m-1\}$. For the main result, we establish a parametrization of nilpotent orbits (under $H$ ) in $\mathfrak{g}^{1}$ in terms of the data arising from the Bruhat-Tits building.

More specifically, for $x \in F_{L}^{*}$, a generalized $(r, L)$-facet, we attach an $\mathfrak{f}$-vector space $V_{F_{L}^{*}}:=\mathfrak{g}_{x, r} / \mathfrak{g}_{x, r^{+}}$to $F_{L}^{*}$. We call a coset $e$ lying in $V_{F_{L}^{*}}$ a Moy-Prasad coset. Such a coset is said to be degenerate if it intersects $\mathcal{N}$, the set of nilpotent elements in $\mathfrak{g}$, nontrivially. At this point, we restrict our attention to degenerate cosets in the eigenspace of weight 1 in $V_{F_{L}^{*}}$, denoted $V_{F_{L}^{*}}^{1}$, and let $I_{r}^{n}$ denote the set of pairs of the form $\left(F_{L}^{*}, e\right)$, with $F_{L}^{*}$ a generalized $(r, L)$-facet and $e \in V_{F_{L}^{*}}^{1}$ a degenerate coset. We define a natural equivalence relation $\sim$ on $I_{r}^{n}$. To each pair $\left(F_{L}^{*}, e\right) \in I_{r}^{n}$, with some restrictions on $\mathbf{G}$ and $k$ described in Chapter IV, we associate a nilpotent $H$-orbit $\mathcal{O}_{1}\left(F_{L}^{*}, e\right)$ in $\mathfrak{g}^{1}$, which (in Chapter $\mathrm{V}$ ) is described as the unique nilpotent $H$-orbit in $\mathfrak{g}^{1}$ of minimal dimension intersecting $e$ nontrivially. Let $\mathcal{O}_{1}(0)$ denote the set of nilpotent $H$-orbits in $\mathfrak{g}^{1}$. Assuming the residual characteristic of $k$ is sufficiently large (see Chapter V) and restricting to a natural subset $I_{r}^{d}$ (the noticed orbits of Definition V.18 of pairs in $I_{r}^{n}$ ) we prove the following theorem:

Theorem I.1. There is a bijective correspondence between $I_{r}^{d} / \sim$ and $\mathcal{O}_{1}(0)$ given by the map that sends $\left(F_{L}^{*}, e\right)$ to $\mathcal{O}_{1}\left(F_{L}^{*}, e\right)$.

Since a motivation for this work comes from the goal of understanding the structure of $p$-adic symmetric spaces, we remark that any reductive, linear algebraic group 
$\mathbf{J}$ defined over a local field can be thought of as a symmetric space in the following way: let $\mathbf{G}=\mathbf{J} \times \mathbf{J}$ and define an involution $\theta$ by $(x, y) \mapsto(y, x)$. Then, the diagonal $\mathbf{H}:=\{(x, x) \mid x \in \mathbf{J}\}$ occurs as the set of $\theta$-fixed points, and we may identify $\mathbf{G} / \mathbf{H}$ with $\mathbf{J}$. This is often referred to as the group (or diagonal) case. Note that $\mathfrak{p}$, as defined earlier, may be identified with the vector space of $k$-rational points of $\operatorname{Lie}(\mathbf{J})$.

For our purposes, we will primarily be concerned with the case where $\mathbf{H}$ is isotropic over $k$. More specifically, we remark that $H$ preserves $\mathfrak{g}^{i}$ under the adjoint action. We say that $X \in \mathfrak{g}^{i}$ is nilpotent whenever there exists a one-parameter subgroup $\lambda \in \mathbf{X}_{*}^{k}(\mathbf{G})$ such that

$$
\lim _{t \rightarrow 0}^{\lambda(t)} X=0 .
$$

It will be demonstrated in Remark IV.16 that, under mild restrictions on the characteristic of $k$, we may assume $\lambda$ lies in $\mathbf{X}_{*}^{k}(\mathbf{H})$.

\subsubsection{Example}

We demonstrate the parametrization for symmetric spaces in the case $r=0$ in the following example. Let $k=\mathbb{Q}_{p}$, with $p \neq 2$. Let $\mathbf{G}=\mathbf{S L}_{3}$, and consider the involution $\theta: \mathbf{S L}_{3} \rightarrow \mathbf{S L}_{3}$ defined by $A \mapsto J\left(A^{t}\right)^{-1} J$, where $J=\left(\begin{array}{ccc}0 & 0 & 1 \\ 0 & 1 & 0 \\ 1 & 0 & 0\end{array}\right)$. Under $d \theta$, the Lie algebra $\mathfrak{s l}_{3}(k)$ decomposes as

$$
\mathfrak{s l}_{3}(k)=\left\{\left(\begin{array}{ccc}
a & b & 0 \\
c & 0 & -b \\
0 & -c & -a
\end{array}\right)\right\} \oplus\left\{\left(\begin{array}{ccc}
x & y & s \\
z & -2 x & y \\
u & z & x
\end{array}\right)\right\} .
$$

As further explained in the appendix, representatives for the six nilpotent $H$-orbits 
in $\mathfrak{p}$ are $\left(\begin{array}{ccc}0 & 0 & 0 \\ 0 & 0 & 0 \\ 0 & 0 & 0\end{array}\right),\left(\begin{array}{lll}0 & 1 & 0 \\ 0 & 0 & 1 \\ 0 & 0 & 0\end{array}\right)$, and $\left\{\left(\begin{array}{ccc}0 & 0 & z \\ 0 & 0 & 0 \\ 0 & 0 & 0\end{array}\right) \mid \bar{z} \in \mathbb{Q}_{p}^{\times} /\left(\mathbb{Q}_{p}^{\times}\right)^{2}\right\}$. The diagonal torus in the set of $\theta$-fixed points, $\mathbf{H}=\mathbf{P G L}_{2}$, is a maximal $k$-split torus $\mathbf{T}$ which lies in the diagonal maximal $k$-split torus $\mathbf{T}^{\prime}$ of $\mathbf{S L}_{3}$. If

$$
t=\left(\begin{array}{ccc}
a & 0 & 0 \\
0 & b & 0 \\
0 & 0 & (a b)^{-1}
\end{array}\right)
$$

is an element of $\mathbf{T}^{\prime}$, define $\alpha$ and $\beta$ by $\alpha(t)=a b^{-1}$ and $\beta(t)=a b^{2}$. Then, $\{\alpha, \beta\}$ is a choice of simple roots of $\mathbf{T}^{\prime}$ in $\mathbf{G}$ with respect to $k$. We let $\check{\alpha}$ and $\check{\beta}$ denote the associated co-roots, respectively.

Using [[26], Theorem 1.9], we are able to identify the building of $H$ with the set of $\theta$-fixed points in $\mathcal{B}(G)$. Thus, we identify the apartment corresponding to the diagonal torus in $\mathbf{P G L}_{2}$ with an affine subspace of the apartment corresponding to the diagonal torus in $\mathbf{S L}_{3}$.

In order to provide a parameterization of the nilpotent $P G L_{2}$-orbits in $\mathfrak{p}$, we restrict our attention to subsets of $\mathcal{B}(H)$ which arise naturally by considering $\theta$-stable facets of $\mathcal{B}(G)$. We call a subset $F$ in an apartment $\mathcal{A} \subset \mathcal{B}(H)$ a $\theta$-facet if $F$ is the set of $\theta$-fixed points of a $\theta$-stable facet $F^{\prime}$ in some apartment of $\mathcal{B}(G)$.

The corresponding apartments are represented in Figure 1, along with the $\theta$-facets arising from the apartment associated to $\mathbf{T}$.

The $\theta$-facets in Figure 1 labelled $F_{1}, F_{2}$, and $F_{3}$ are those which arise as the fixed points of facets in the closure of a fixed alcove $C$ of $\mathcal{A}\left(\mathbf{T}^{\prime}, \mathbb{Q}_{p}\right)$. In particular, $F_{1}$ is the vertex at the base of $\bar{C}, F_{2}$ is the $\theta$-facet arising from $C$ itself, and $F_{3}$ is the point in the closure of the alcove whose lift is the segment at the top of $\bar{C}$.

If $F$ is a $\theta$-facet containing some point $x \in \mathcal{B}(H)$, we note that $\theta$ induces a map, 


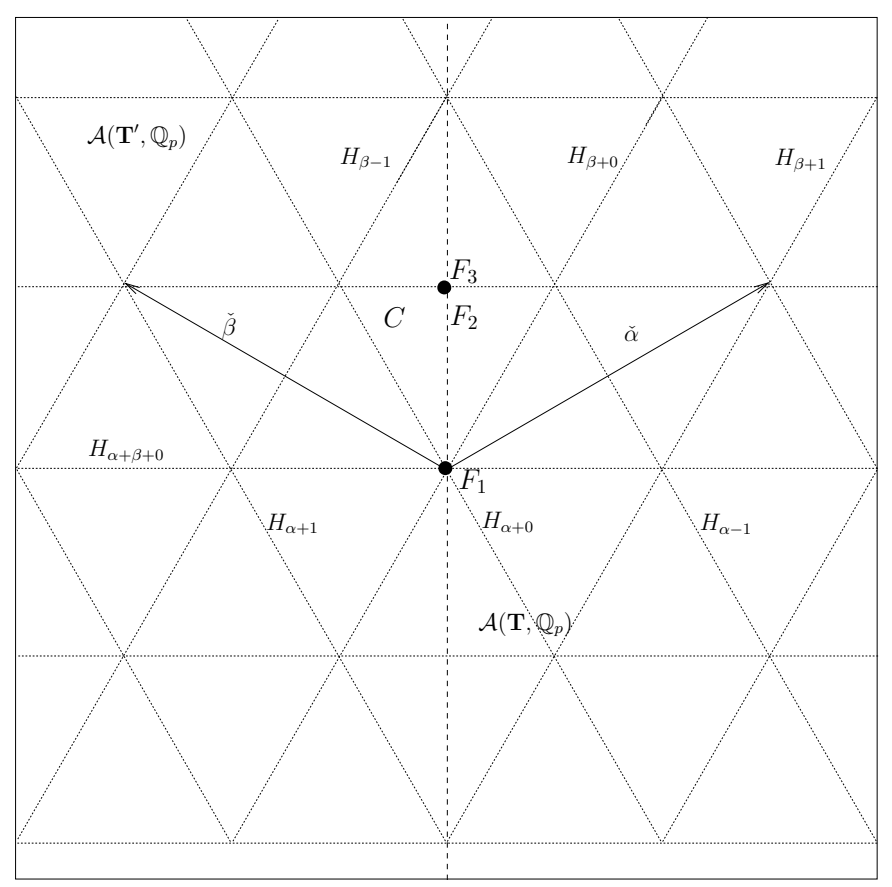

Figure 1.1: Affine apartments of $G=\mathbf{S L}_{3}\left(\mathbb{Q}_{p}\right)$ and $H=\mathbf{P G L}_{2}\left(\mathbb{Q}_{p}\right)$ (dotted line represents $\mathcal{A}\left(\mathbf{T}, \mathbb{Q}_{p}\right)$ )

which we denote $d \theta_{F}$, on the Lie algebra $V_{F}:=\mathfrak{g}_{x} / \mathfrak{g}_{x}^{+}$. In this way, we may consider the decomposition of $V_{F}$ under $d \theta_{F}$, and examine nilpotent $H$-orbits in the $(-1)$ eigenspace of $V_{F}$. The corresponding Lie algebras associated to each of these $\theta$-facets are listed below:

$$
\begin{aligned}
& V_{F_{1}}=\left\{\left(\begin{array}{ccc}
a & b & 0 \\
c & 0 & -b \\
0 & -c & -a
\end{array}\right)\right\} \oplus\left\{\left(\begin{array}{ccc}
x & y & s \\
z & -2 x & y \\
u & z & x
\end{array}\right)\right\} \\
& V_{F_{2}}=\left\{\left(\begin{array}{ccc}
a & 0 & 0 \\
0 & 0 & 0 \\
0 & 0 & -a
\end{array}\right)\right\} \oplus\left\{\left(\begin{array}{ccc}
x & 0 & 0 \\
0 & -2 x & 0
\end{array}\right)\right\} \\
& V_{F_{3}}=\left\{\left(\begin{array}{ccc}
a & 0 & 0 \\
0 & 0 & 0 \\
0 & 0 & x
\end{array}\right)\right\} \oplus\left\{\left(\begin{array}{ccc}
x & 0 & p^{-1} s \\
0 & -2 x & 0 \\
0 & 0 & -a
\end{array}\right)\right\},
\end{aligned}
$$

with all lowercase entries being representatives in $\mathbb{Z}_{p}$. 
At this point, we would like to match up nilpotent $H$-orbits with nilpotent orbits arising from each of the above $\mathfrak{f}$-Lie algebras. In order to obtain a bijection, however, we must restrict ourselves to elements $e \in V_{F}^{-}$whose centralizer (in $V_{F}^{+}$) does not contain certain noncentral (meaning elements in $V_{F}^{+}$which do not belong to the center of $V_{F}$ ) semisimple elements which are fixed by $\theta$. This may be thought of as a restriction on the type of Levi subalgebra which is allowed to contain $e$ and thus resembles the distinguished condition found in [[12], Remark 5.5.2]. We call such nilpotent elements noticed.

The noticed nilpotent $H$-orbits in $V_{F_{1}}^{-}$have representatives of the form

$$
\left\{\left(\begin{array}{lll}
0 & 1 & 0 \\
0 & 0 & 1 \\
0 & 0 & 0
\end{array}\right)\right\}
$$

and

$$
\left\{\left(\begin{array}{ccc}
0 & 0 & s \\
0 & 0 & 0 \\
0 & 0 & 0
\end{array}\right) \mid \bar{s} \in \mathbb{F}_{p}^{\times} /\left(\mathbb{F}_{p}^{\times}\right)^{2}\right\} .
$$

The only noticed nilpotent $H$-orbit in $V_{F_{2}}^{-}$is the trivial orbit. There are two more noticed nilpotent $H$-orbits lying in $V_{F_{3}}^{-}$whose lifts modulo $p^{-1}$ correspond to the two representatives of square classes in $\mathbb{F}_{p}^{\times} /\left(\mathbb{F}_{p}^{\times}\right)^{2}$. Upon taking lifts, these six orbits clearly match up with the six nilpotent $H$-orbits in $\mathfrak{p}$ discussed above. 


\section{CHAPTER II}

\section{Algebraic groups over local fields}

\subsection{Algebraic groups, finite group actions, and associated notation}

Let $k$ be a field with a nontrivial discrete valuation $\nu$, and let $K$ be a maximal unramified extension of $k$. Let $R$ (resp. $R_{K}$ ) denote the ring of integers in $k$ (resp. $K$ ), and let $\mathfrak{f}$ (resp. $\mathfrak{F}$ ) denote the residue field of $k$ (resp. $K$ ). We assume $k$ is complete and that $\mathfrak{f}$ is perfect. Let $\mathbf{G}$ be a reductive, linear algebraic group defined over $k$, and let $L$ be a finite group which acts on $\mathbf{G}$ by algebraic automorphisms defined over $k$. Let $\mathbf{G}^{\circ}$ denote the neutral component of $\mathbf{G}$.

We fix a uniformizer $\varpi$ of $k$, with respect to $\nu$, and let $M$ denote the minimal Galois extension of $K$ over which $\mathbf{G}^{\circ}$ splits. Let $\ell=[M: K]$. If $\nu$ also denotes the extension of $\nu$ to $M$, then we normalize $\nu$ so that $\nu\left(M^{\times}\right)=\mathbb{Z}$.

If $k^{\prime}$ is any field, we let $\overline{k^{\prime}}$ denote an algebraic closure of $k^{\prime}$. Suppose $\mathbf{C}$ is a linear algebraic group defined over $k^{\prime}$. We will identify $\mathbf{C}$ with the $\overline{k^{\prime}}$-points of $\mathbf{C}$. If $J$ is a finite group acting on $\mathbf{C}$ by $k^{\prime}$-automorphisms, we will almost always let $\mathbf{C}^{J}$ denote the set $\{x \in \mathbf{C} \mid j \cdot x=x$ for all $j \in J\}$; in the case that $\mathbf{C}$ is a $J$-stable torus, we will let $\mathbf{C}^{J}$ be the connected component of this set. Lastly, if $C$ is any group, we let $[C, C]$ denote its derived subgroup, and if $\mathfrak{c}$ is any Lie algebra, we let $[\mathfrak{c}, \mathfrak{c}]$ denote its 
derived subalgebra.

Let $\mathbf{H}$ be a subgroup of $\mathbf{G}$ with $\left(\mathbf{G}^{L}\right)^{\circ} \subset \mathbf{H} \subset \mathbf{G}^{L}$ such that $\mathbf{H}$ is defined over $k$. By [[26], 2.4], $\mathbf{H}^{\circ}$ is reductive. We let $G$ denote the group of $k$-rational points of $\mathbf{G}$ and similarly let $H$ denote the group of $k$-rational points of $\mathbf{H}$. Each element $l \in L$ induces a $k$-automorphism, which we denote $d l$, on the Lie algebra, $\mathfrak{g}:=\operatorname{Lie}(\mathbf{G})$, of G. We will assume that $\mathfrak{g}$, the vector space of $k$-rational points of $\mathfrak{g}$, is completely reducible as a representation of $L$ (this always happens, for example, when $L$ is cyclic of order 2), and we will write the decomposition as

$$
\mathfrak{g}=\mathfrak{h}+\bigoplus_{\sigma} \mathfrak{g}^{\sigma}
$$

where $\mathfrak{g}^{\sigma}$ is the isotypic component corresponding to some irreducible representation $\sigma$ of $L$ contained in $\mathfrak{g}$, and $\mathfrak{h}$ is the set of elements in $\mathfrak{g}$ on which $L$ acts trivially. In Chapters IV and V, where $L$ is a finite cyclic group of order $m$, the isotypic components are eigenspaces in $\mathfrak{g}$, and if $\theta$ is a generator, we may write the decomposition as

$$
\mathfrak{g}=\mathfrak{h} \oplus \bigoplus_{i=1}^{m-1} \mathfrak{g}^{i}
$$

where $\mathfrak{g}^{i}=\left\{X \in \mathfrak{g} \mid \theta(X)=\xi^{i} X\right\}$, and $\xi$ is a primitive $m^{\text {th }}$ root of unity. Generally, if $V$ is a $k^{\prime}$-vector space on which some finite group $J$ acts by $k^{\prime}$-automorphisms, we let $V^{J}$ denote the set $\{X \in V \mid j \cdot X=X$ for all $j \in J\}$.

When we refer to a Levi subgroup of $\mathbf{G}$ (resp. $\mathbf{H}$ ), we mean a Levi subgroup of $\mathbf{G}^{\circ}$ (resp. $\mathbf{H}^{\circ}$ ). We apply the same terminology to tori and parabolic subgroups.

Let Ad denote the adjoint action of $G$ on $\mathfrak{g}$. For $g \in G$ and $X \in \mathfrak{g}$, let ${ }^{g} X=$ $\operatorname{Ad}(g)(X)$. Suppose $\mathbf{M}$ is a linear algebraic group defined over $k^{\prime}$ acting on its Lie algebra $\mathfrak{m}$ via the adjoint action. If $m \in \mathbf{M}$, we will let $\operatorname{Int}(m)$ denote conjugation by $m$. Let $M \supset J$ and $\mathfrak{j}$ be subsets of $\mathbf{M}$ and $\mathfrak{m}$ respectively. Then, let $C_{M}(\mathfrak{j})=\{m \in$ 
$M \mid{ }^{m} X=X$ for all $\left.X \in \mathfrak{j}\right\}$. Similarly, we let $N_{M}(\mathfrak{j})=\left\{m \in M \mid{ }^{m} \mathfrak{j}=\mathfrak{j}\right\}$.

Although we restrict ourselves to discussing nilpotent elements which will lie in

$\mathfrak{g}^{1}$ (under the action of a finite cyclic group), our definition of nilpotence will be as in [[12]]. In particular, we call $X \in \mathfrak{g}^{1}$ nilpotent provided there exists some $\lambda \in \mathbf{X}_{*}^{k}(\mathbf{G})$ such that $\lim _{t \rightarrow 0}{ }^{\lambda(t)} X=0$. We let $\mathcal{N}$ denote the set of nilpotent elements in $\mathfrak{g}$ and define $\mathcal{N}^{1}:=\mathcal{N} \cap \mathfrak{g}^{1}$. We also let $\mathcal{U}$ denote the set of unipotent elements in $G$.

If the residue field $\mathfrak{f}$ has positive characteristic, we denote the characteristic of $\mathfrak{f}$ by $p$. If the residue field has characteristic zero, we let $p=\infty$.

\subsection{The Bruhat-Tits building, apartments, and $L$-fixed points}

We let $\mathcal{B}(G)$ denote the (enlarged) Bruhat-Tits building of $\mathbf{G}^{\circ}(k)$, and, similarly, let $\mathcal{B}(H)$ denote the (enlarged) Bruhat-Tits building of $\mathbf{H}^{\circ}(k)$. Unless otherwise stated, the symbol $\mathcal{B}(G)$ (resp. $\mathcal{B}(H)$ ) will always refer to the enlarged Bruhat-Tits building; that is, it takes the center of $\mathbf{G}^{\circ}(k)$ (resp. $\left.\mathbf{H}^{\circ}(k)\right)$ into account. We note that since $K / k$ is a maximal unramified extension, $\mathfrak{F}$ is an algebraic closure of $\mathfrak{f}$, and $\mathcal{B}(G)$ can be identified with the $\operatorname{Gal}(K / k)$-fixed points of $\mathcal{B}(\mathbf{G}, K)$, the Bruhat-Tits building of $\mathbf{G}^{\circ}(K)$.

If $\mathbf{S}$ (resp. $\mathbf{S}^{\prime}$ ) is a maximal $k$-split torus of $\mathbf{H}$ (resp. $\mathbf{G}$ ), we will let $\mathcal{A}(\mathbf{S}, k)$ (resp. $\left.\mathcal{A}\left(\mathbf{S}^{\prime}, k\right)\right)$ denote the associated apartment in $\mathcal{B}(H)$ (resp. $\left.\mathcal{B}(G)\right)$. If $\mathcal{A}$ is an apartment in $\mathcal{B}(H)$, and $\Omega$ is a subset of $\mathcal{A}$, we let $A(\Omega, \mathcal{A})$ denote the smallest affine subspace of $\mathcal{A}$ containing $\Omega$. We let dist: $\mathcal{B}(G) \times \mathcal{B}(G) \rightarrow \mathbb{R}_{+}$denote a nontrivial $G$-invariant distance function as described in $[[31], 2.3]$. For $x, y \in \mathcal{B}(G)$, let $[x, y]$ denote the geodesic in $\mathcal{B}(G)$ from $x$ to $y$ with respect to dist. Let $(x, y]$ denote $[x, y] \backslash\{x\}$.

For $x \in \mathcal{B}(\mathbf{G}, K)$, we let $\mathbf{G}(K)_{x}$ and $\mathbf{G}(K)_{x}^{+}$denote the parahoric subgroup associated to $x$ and its pro-unipotent radical, respectively. The groups $\mathbf{G}(K)_{x}$ and $\mathbf{G}(K)_{x}^{+}$ only depend on the facet in $\mathcal{B}(\mathbf{G}, K)$ containing $x$. Therefore, if $F \subset \mathcal{B}(\mathbf{G}, K)$ is the facet containing $x$, we define $\mathbf{G}(K)_{F}$ and $\mathbf{G}(K)_{F}^{+}$to be $\mathbf{G}(K)_{x}$ and $\mathbf{G}(K)_{x}^{+}$, re- 
spectively. When $F$ is $\operatorname{Gal}(K / k)$-stable, the quotient $\mathbf{G}(K)_{F} / \mathbf{G}(K)_{F}^{+}$is the group of $\mathfrak{F}$-points of a connected, reductive group $\mathrm{G}_{F}$ defined over $\mathfrak{f}$.

If $x \in \mathcal{B}(G)$, we denote the parahoric associated to $x$ and its pro-unipotent radical by $G_{x}$ and $G_{x}^{+}$, respectively. We recall that these subgroups are obtained as the sets of $\operatorname{Gal}(K / k)$-fixed points of parahorics defined over the maximal unramified extension. That is, when $x$ lies in $\mathcal{B}(G)$, we have $G_{x}:=\mathbf{G}(K)_{x}^{\operatorname{Gal}(K / k)}$ and $G_{x}^{+}:=\left(\mathbf{G}(K)_{x}^{+}\right)^{\operatorname{Gal}(K / k)}$. The quotient $G_{x} / G_{x}^{+}$coincides with the group of f-rational points of the connected, reductive group $\mathrm{G}_{x}$ defined over $\mathfrak{f}$. Moreover, we have $\mathrm{G}_{x}(\mathfrak{f})=\mathrm{G}_{x}(\mathfrak{F})^{\mathrm{Gal}(\overline{\mathfrak{f}} / \mathfrak{f})}$.

If $\mathbf{S}$ (resp. $\mathbf{S}^{\prime}$ ) is a maximal $k$-split torus in $\mathbf{H}$ (resp.G), we let $\Phi=\Phi(\mathbf{S}, k)$ $\left(\operatorname{resp} . \Phi\left(\mathbf{S}^{\prime}, k\right)\right)$ denote the set of roots of $\mathbf{S}$ (resp. $\left.\mathbf{S}^{\prime}\right)$ in $\mathbf{H}$ (resp.G) with respect to $k$. If $\mathcal{A}\left(\operatorname{resp} . \mathcal{A}^{\prime}\right)$ is the apartment corresponding to $\mathbf{S}\left(\operatorname{resp} . \mathrm{S}^{\prime}\right)$, let $\Psi=\Psi(\mathcal{A})\left(\operatorname{resp} . \Psi\left(\mathcal{A}^{\prime}\right)\right)$ denote the set of affine roots of $\mathbf{H}$ (resp.G) with respect to $\mathbf{S}, k$ and $\nu$ (resp. $\mathbf{S}^{\prime}, k$ and $\nu$ ). If $\psi \in \Psi$ is an affine root, we let $\dot{\psi} \in \Phi$ denote the gradient of $\psi$. Whenever $\psi$ is an affine root of $\mathbf{H}$ (resp. $\mathbf{G}$ ) with respect to $\mathbf{S}$ (resp. $\mathbf{S}^{\prime}$ ) and $\Omega$ is a subset of the apartment associated to $\mathbf{S}$ (resp. $\mathbf{S}^{\prime}$ ), we let $\operatorname{res}_{\Omega} \psi$ denote the restriction of $\psi$ to $\Omega$.

Throughout this thesis, we will abuse notation and let $l \in L$ also denote the induced map on the building of $G$. We shall also assume that $p$ does not divide the order of $L$. Under this assumption, Prasad and $\mathrm{Yu}$ proved that $\mathcal{B}(H)$ can be identified with $\mathcal{B}(G)^{L}$. Whenever $\Omega^{\prime}$ is a subset of $\mathcal{B}(G)$, we will define $\Omega^{\prime L}:=\left\{x \in \Omega^{\prime} \mid l(x)=\right.$ $x$ for all $l \in L\}$.

\subsection{The Moy-Prasad filtrations}

Let $x \in \mathcal{B}(H)$ and $r \in \mathbb{R}$. We let $\mathfrak{g}_{x, r}$ denote the Moy-Prasad filtration of depth $r$ as defined in [[21], Section 3.2]. As we will show in Chapter III, the filtration lattices $\mathfrak{g}_{x, r}$ and $\mathfrak{g}_{x, r^{+}}$are $L$-stable and thus each element of $L$ induces an $\mathfrak{f}$-endomorphism of the $\mathfrak{f}$-vector space $\mathfrak{g}_{x, r} / \mathfrak{g}_{x, r^{+}}$. 
Let $\mathbf{S}$ be a maximal $k$-split torus in $\mathbf{H}$, and suppose $\mathbf{T}$ is a maximal $K$-split $k$ torus in $\mathbf{H}$ containing $\mathbf{S}$. By [[26], Theorem 1.9], we can choose a maximal $k$-split torus $\mathbf{S}^{\prime}$ of $\mathbf{G}$ containing $\mathbf{S}$ and a maximal $K$-split $k$-torus $\mathbf{T}^{\prime}$ of $\mathbf{G}$ containing $\mathbf{S}^{\prime}$ and $\mathbf{T}$. Since $\mathbf{G}$ is quasi-split over $K$, we know that $\mathbf{Z}^{\prime}:=C_{\mathbf{G}^{\circ}}\left(\mathbf{T}^{\prime}\right)$ is a maximal $k$-torus in $\mathbf{G}$ containing $\mathbf{T}^{\prime}$. We will define $\mathbf{Z}$ to be $C_{\mathbf{H}^{\circ}}(\mathbf{T})$, which is a maximal $k$-torus of $\mathbf{H}$.

Let $\mathfrak{z}^{\prime}$ denote the Lie algebra of $\mathbf{Z}^{\prime}$. Following [[21], 3.2], there is a filtration of $\mathfrak{z}^{\prime}(K)$ for each $r \in \mathbb{R}$ which we denote $\mathfrak{z}^{\prime}(K)_{r}$. Moreover, for each affine functional $\psi \in \Psi\left(\mathcal{A}\left(\mathbf{T}^{\prime}, K\right)\right)$, there exists a lattice denoted $\mathfrak{u}_{\psi}$, which lies in the root space in $\mathfrak{g}(K)$ with respect to $\mathbf{T}, \mathbf{G}$, and $\dot{\psi}$. The lattice $\mathfrak{g}(K)_{x, r}$ is defined as the the $R_{K^{-}}$ submodule of $\mathfrak{g}(K)$ spanned by $\mathfrak{z}^{\prime}(K)_{r}$ and the $\mathfrak{u}_{\psi}$ 's for which $\psi(x) \geq r$.

\subsubsection{A result of Prasad-Yu}

In order to discuss a type of facet which takes both $L$ and the facet structure of $\mathcal{B}(G)$ into account, it will be useful to discuss the relationship between apartments in $\mathcal{B}(H)$ and those in $\mathcal{B}(G)$. Prasad and Yu have shown in [[26], Theorem 1.9] that there is an $\mathbf{H}^{\circ}(k)$-equivariant map $\iota: \mathcal{B}(H) \rightarrow \mathcal{B}(G)$ such that the image is $\mathcal{B}(G)^{L}$, uniquely defined up to translation by $\mathbf{X}_{*}(\mathbf{C}) \otimes \mathbb{R}$, where $\mathbf{C}$ is the maximal $k$-split torus in the center of $\mathbf{H}$. Moreover, for every maximal $k$-split torus $\mathbf{S}$ of $\mathbf{H}$, there is a maximal $k$-split torus $\mathbf{S}^{\prime}$ of $\mathbf{G}$ such that $\mathcal{A}(\mathbf{S}, k)$ is mapped into $\mathcal{A}\left(\mathbf{S}^{\prime}, k\right)$ by an affine transformation. In [[26], Lemma 1.9.3], it is shown that such a map is compatible with unramified base change. In particular, there is an $\mathbf{H}^{\circ}(K)$-equivariant map $\iota_{K}: \mathcal{B}(\mathbf{H}, K) \rightarrow \mathcal{B}(\mathbf{G}, K)$ which is also $\operatorname{Gal}(K / k)$-equivariant, such that the restriction of $\iota_{K}$ to $\mathcal{B}(H)$ shares the same properties as $\iota$.

Let $\hat{K}$ denote the analytic completion of $K$ in $\bar{k}$. When $J$ is a finite cyclic group of $k$-automorphisms of $\mathbf{G}$, and $\mathbf{H}^{\prime}=\left(\mathbf{G}^{J}\right)^{\circ}$, it is shown in [[26], Remark 1.5.4] that every maximal $\hat{K}$-split torus in $\mathbf{H}^{\prime}$ is contained in some $J$-stable maximal $\hat{K}$-split torus of G. This remarkable fact is false over $k$ for $m \geq 3$. However, in the special case when 
$|J|=2$, Helminck and Wang (in [[14]] for any field $k$ of odd characteristic) show that every maximal $k$-split torus in $\mathbf{H}$ is contained inside a $J$-stable maximal $k$-split torus in G. This fact is also shown in [[17], Corollary 5.7(i)] when $k$ is a $p$-adic field and in [[17], Proposition 3.4.1] when $k$ is a finite field of odd characteristic. When $J$ is cyclic of order exceeding 2, a counterexample is given below which generalizes to $\mathbf{S L}_{n}$.

Example II.1. Let $k=\mathbb{Q}_{p}$ and $\mathbf{G}=\mathbf{S L}_{2}$. Let $\epsilon \notin\left(R^{\times}\right)^{2}$, and let $x$ be the Teichmuller lift of an element of order $p+1$ in the torus

$$
\mathbf{T}=\left\{\left(\begin{array}{cc}
a & b \\
b \epsilon & a
\end{array}\right) \mid a^{2}-b^{2} \epsilon=1\right\},
$$

for which $x^{2}$ is not $\{ \pm 1\} \subset S L_{2}\left(\mathbb{Q}_{p}\right)$. We note that the torus in $\mathbf{G}$ corresponding to $\mathbf{T}$ is a $k$-minisotropic (see [[10]]) maximal torus and that $x$ is regular. In particular, the centralizer of $x$ is $\mathbf{T}$ and this is the only maximal torus containing $x$. Let the finite group $J:=\langle x\rangle$ act on $\mathbf{S L}_{2}$ by conjugation. Then, clearly $\mathbf{T}$ is $J$-stable. Moreover, if a maximal $k$-split torus $\mathbf{S}$ is $J$-stable, then $x^{2} \in \mathbf{S}$, since $\mathbf{S}$ is maximal. However, this implies $x^{2} \in \mathbf{T}(k) \cap \mathbf{S}(k)$, a contradiction since $\mathbf{T}$ is $k$-minisotropic and $x^{2} \notin\{ \pm 1\}$.

Remark II.2. It is not true, in general, that an $L$-stable apartment of $\mathcal{B}(G)$ gives rise to an apartment in $\mathcal{B}(H)$. Consider $\mathbf{S L}_{2}$ and the involution $\theta$ defined by $X \mapsto\left(X^{t}\right)^{-1}$. Supposing $-1 \in\left(\mathbb{Q}_{p}^{\times}\right)^{2}$, the fixed points under this involution consist of a maximal $k$-split torus. However, the diagonal torus is a $\theta$-stable maximal $k$-split torus in $\mathbf{G}$ whose set of $\theta$-fixed points is $\{ \pm 1\}$. 


\section{CHAPTER III}

\section{Finite group actions on the Bruhat-Tits building}

\section{1 ( $r, L)$-facets and Moy-Prasad lattices}

Throughout this chapter, we assume that $L$ is a finite group of $k$-automorphisms of $\mathbf{G}$.

Fix an apartment $\mathcal{A}^{\prime}$ of $\mathcal{B}(G)$. For $\psi \in \Psi\left(\mathcal{A}^{\prime}\right)$, define the hyperplane

$$
H_{\psi-r}:=\left\{x \in \mathcal{A}^{\prime} \mid \psi(x)=r\right\}
$$

As in [[12], Section 3.1], we call a nonempty subset $F^{\prime} \subset \mathcal{A}^{\prime}$ an $r$-facet of $\mathcal{A}^{\prime}$ if there is some finite subset $S \subset \Psi\left(\mathcal{A}^{\prime}\right)$ for which

1. $F^{\prime} \subset H_{S}:=\bigcap_{\psi \in S} H_{\psi-r}$

2. $F^{\prime}$ is a connected component (in $\left.H_{S}\right)$ of $H_{S} \backslash \bigcup_{\psi \in \Psi\left(\mathcal{A}^{\prime}\right) \backslash S}\left(H_{S} \cap H_{\psi-r}\right)$.

If $F^{\prime}$ is an $r$-facet in $\mathcal{A}^{\prime}$, we define its dimension to be the dimension of $A\left(F^{\prime}, \mathcal{A}^{\prime}\right)$.

The following remark, which is a consequence of the definitions above, will be important for later discussion of $L$-stable $r$-facets.

Remark III.1. If $F_{1}^{\prime}, F_{2}^{\prime}$ are $r$-facets in $\mathcal{A}^{\prime}$, and $F_{2}^{\prime} \cap A\left(F_{1}^{\prime}, \mathcal{A}^{\prime}\right) \neq \emptyset$, then $F_{2}^{\prime}$ is entirely 
contained in $A\left(F_{1}^{\prime}, \mathcal{A}^{\prime}\right)$. To see why this is true, write

$$
A\left(F_{1}^{\prime}, \mathcal{A}^{\prime}\right)=H_{S}:=\bigcap_{\psi \in S} H_{\psi-r}
$$

where $S$ is finite and $F_{1}^{\prime}$ is a connected component of $H_{S} \backslash \bigcup_{\psi \in \Psi\left(\mathcal{A}^{\prime}\right) \backslash S}\left(H_{S} \cap H_{\psi-r}\right)$. Write

$$
A\left(F_{2}^{\prime}, \mathcal{A}^{\prime}\right)=H_{S^{\prime}}:=\bigcap_{\rho \in S^{\prime}} H_{\rho-r}
$$

where $S^{\prime}$ is finite and $F_{2}^{\prime}$ is a connected component of $H_{S^{\prime}} \backslash \bigcup_{\rho \in \Psi\left(\mathcal{A}^{\prime}\right) \backslash S^{\prime}}\left(H_{S^{\prime}} \cap H_{\rho-r}\right)$.

It will be enough to show that $S \subset S^{\prime}$. If $S$ is empty, then the statement is obviously true, so suppose $\psi \in S \backslash S^{\prime}$. Let $x_{2} \in F_{2}^{\prime} \cap A\left(F_{1}^{\prime}, \mathcal{A}^{\prime}\right)$. Then, since $F_{2}^{\prime}$ lies in $H_{S^{\prime}} \backslash \bigcup_{\rho \in \Psi\left(\mathcal{A}^{\prime}\right) \backslash S^{\prime}}\left(H_{S^{\prime}} \cap H_{\rho-r}\right)$, we must have $x_{2} \in \mathcal{A}^{\prime} \backslash H_{\psi-r}$. But, since $x_{2} \in A\left(F_{1}^{\prime}, \mathcal{A}^{\prime}\right)$, we have $\psi\left(x_{2}\right)=r$, a contradiction. Thus, we must have $S \subset S^{\prime}$, that is, $F_{2}^{\prime}$ is entirely contained in $A\left(F_{1}^{\prime}, \mathcal{A}^{\prime}\right)$.

Definition III.2. Define an $(r, L)$-facet to be a nonempty subset $F$ in an apartment $\mathcal{A} \subset \mathcal{B}(H)$ such that there exists an apartment $\mathcal{A}^{\prime} \subset \mathcal{B}(G)$ and an $r$-facet $F^{\prime} \subset \mathcal{A}^{\prime}$ with $F=F^{\prime L}$.

Remark III.3. In Definition III.2, we have defined a structure on apartments in $\mathcal{B}(H)$ which is finer than the $r$-facet structure of apartments in $\mathcal{B}(H)$. For example, take $r=0, \mathbf{G}=\mathbf{S L}_{2}$ equipped with the involution $\theta(A)=J\left(A^{t}\right)^{-1} J$ as in Example 1.3.0.1. From Figure 1.1, we see that $\theta$-facet $F_{2}$ is a strictly smaller subset of the $H$-alcove of $\mathcal{B}(H)$ that has boundary $\left\{F_{1}, F_{1}+(\check{\alpha}+\check{\beta})\right\}$

Definition III.4. Let $F$ be an $(r, L)$-facet in an apartment $\mathcal{A} \subset \mathcal{B}(H)$. Define the dimension of an $(r, L)$-facet $F$ by

$$
\operatorname{dim} F:=\operatorname{dim} A(F, \mathcal{A})
$$


Let $\mathcal{A}^{\prime}$ be an apartment of $\mathcal{B}(G)$. Suppose $F$ is an $(r, L)$-facet which lies inside an $r$-facet $F^{\prime} \subset \mathcal{A}^{\prime}$, and let $x, y \in F$. Then, in particular, since $x$ and $y$ lie inside the $r$-facet $F^{\prime}$, we have $\mathfrak{g}_{x, r}=\mathfrak{g}_{y, r}$ and $\mathfrak{g}_{x, r^{+}}=\mathfrak{g}_{y, r^{+}}$. A proof of this statement can be found in [[12], 3.1.4]. This allows us to make the following definition.

Definition III.5. Let $F$ be an $(r, L)$-facet of $\mathcal{A}$. Fix $x \in F$. Set

$$
\mathfrak{g}_{F}:=\mathfrak{g}_{x, r}
$$

and

$$
\mathfrak{g}_{F}^{+}:=\mathfrak{g}_{x, r^{+}} .
$$

Lemma III.6. Let $F$ be an $(r, L)$-facet of $\mathcal{A} \subset \mathcal{B}(H)$. Suppose $x \in \mathcal{A}$. Then $x \in F$ if and only if $\mathfrak{g}_{x, r}=\mathfrak{g}_{F}$ and $\mathfrak{g}_{x, r^{+}}=\mathfrak{g}_{F}^{+}$.

Proof. We apply the analogous result in [[12], 3.1.4]. If $x \in F$, then, by definition, we have $\mathfrak{g}_{x, r}=\mathfrak{g}_{F}$ and $\mathfrak{g}_{x, r^{+}}=\mathfrak{g}_{F}^{+}$. Suppose $F=F^{\prime L}$, where $F^{\prime}$ is an $r$-facet in an apartment $\mathcal{A}^{\prime} \subset \mathcal{B}(G)$. Then, by [[12], Lemma 3.1.4], since $\mathfrak{g}_{x, r}=\mathfrak{g}_{F}=\mathfrak{g}_{F^{\prime}}$ and $\mathfrak{g}_{x, r^{+}}=\mathfrak{g}_{F}^{+}=\mathfrak{g}_{F^{\prime}}^{+}$, we have $x \in F^{\prime} \cap \mathcal{A}=F$.

Before showing that there is a reasonable decomposition of the Moy-Prasad lattice $\mathfrak{g}_{x, r}$ with respect to the $L$-action, we demonstrate the relationship between the MoyPrasad lattices $\mathfrak{h}_{x, r}$ and $\mathfrak{g}_{x, r}$. The statements we make when discussing these lattices make sense because of [[26], Theorem 1.9]. Let $\mathfrak{g}_{x}$ and $\mathfrak{h}_{x}$ denote the lattices $\mathfrak{g}_{x, 0}$ and $\mathfrak{h}_{x, 0}$, respectively. In the next paragraph, we will show

$$
\mathfrak{g}_{x} \cap \mathfrak{h}=\mathfrak{h}_{x},
$$

so, we may (and do) assume that both $\mathbf{G}$ and $\mathbf{H}$ are connected. 
Let $x \in \mathcal{B}(H)$. Bruhat and Tits in [[5]] have attached a smooth, connected, affine $R$-group scheme $\mathcal{G}_{x}$ to $x$ characterized by

1. The generic fiber of $\mathcal{G}_{x}$ is $\mathbf{G}$

2. The $R_{K}$-points of $\mathcal{G}_{x}$, denoted $\mathcal{G}_{x}\left(R_{K}\right)$, coincide with the parahoric subgroup $\mathbf{G}(K)_{x}$.

Consider the parahoric subgroups $\mathbf{H}(K)_{x}$ and $\mathbf{G}(K)_{x}$. It is clear that

$$
\mathbf{H}(K)_{x} \subset \operatorname{stab}_{\mathbf{G}(K)}(x) .
$$

By the argument given in [[26], Prop 1.7], we must have $\mathbf{H}(K)_{x} \subset \mathbf{G}(K)_{x} \cap \mathbf{H}(K)$. On other other hand, since $x$ is $L$-fixed, (the restriction of) each element $l: \mathbf{G}(K)_{x} \rightarrow$ $\mathbf{G}(K)_{x}$ induces an $R_{K}$-scheme homomorphism of the smooth, affine $R$-group scheme associated to $\mathbf{G}(K)_{x}$, which we denote $\mathcal{G}_{x}$. (see [[5], 1.7.6]). Call the $L$-fixed points of this group scheme $\mathcal{G}_{x}^{L}$ (see [[36], 2.11] and [[8], Proposition A.8.10]). Since $\mathbf{H}(K)_{x} \subset$ $\mathbf{G}(K)_{x}^{L}$, we have an induced $R_{K}$-scheme morphism from $\mathcal{H}_{x}$ to $\mathcal{G}_{x}^{L}$, where $\mathcal{H}_{x}$ is the smooth, connected, affine $R$-group scheme associated to the parahoric subgroup $\mathbf{H}(K)_{x}$. Let $\mathcal{H}$ be the smooth, affine (not necessarily connected) $R$-group scheme associated to $\operatorname{stab}_{\mathbf{H}(K)}(x)$. Then, we have an inclusion

$$
\mathcal{G}_{x}^{L} \subset \mathcal{H} \text {. }
$$

Note that the group scheme $\mathcal{H}_{x}$ is the neutral component of $\mathcal{H}$. In particular, the lattices $\mathfrak{h}(K)_{x}=\operatorname{Lie}\left(\mathcal{H}_{x}\right) \otimes_{R} R_{K}, \operatorname{Lie}\left(\mathcal{G}_{x}^{L}\right) \otimes_{R} R_{K}$, and $\operatorname{Lie}(\mathcal{H}) \otimes_{R} R_{K}$ coincide. By [[8], Prop. A.8.10], we have $\operatorname{Lie}\left(\mathcal{G}_{x}^{L}\right) \otimes_{R} R_{K}=\left(\operatorname{Lie}\left(\mathcal{G}_{x}\right) \otimes_{R} R_{K}\right)^{L}=\mathfrak{g}(K)_{x} \cap \mathfrak{h}(K)$. Thus, since $x$ is $\operatorname{Gal}(K / k)$-fixed, this gives us the equality $\mathfrak{h}_{x}=\mathfrak{g}_{x} \cap \mathfrak{h}$.

Lemma III.7. Let $x \in \mathcal{B}(H)$. Then, we have 


$$
\mathfrak{g}_{x, r} \cap \mathfrak{h}=\mathfrak{h}_{x, r}
$$

Proof. For this proof only, if $M / k$ is a finite extension, let $\operatorname{ord}(M)$ denote the image $\nu\left(M^{\times}\right)$, where $\nu$ denotes the extension of $\nu$ to $M$. Following the proof of [[35], Lemma 8.2], we will first assume that $r \in \operatorname{ord}(k)$. If $\pi_{r}$ is an element of $k$ with valuation $-r$, then $\pi_{r} \mathfrak{g}_{x, r}=\mathfrak{g}_{x, 0}=\mathfrak{g}_{x}$, so the result follows from the observation preceding this proof.

If $r \in \operatorname{ord}(k) \otimes_{\mathbb{Z}} \mathbb{Q}$, we use [[1], 1.4.1] to reduce the statement to the one above. The statement of the proposition now follows by noting that for any real number $r$, we have

$$
\mathfrak{g}_{x, r}=\bigcap_{s<r, s \in \mathbb{Q}} \mathfrak{g}_{x, s}
$$

The previous lemma shows that two points $x$ and $y$ which lie in the same $(r, L)$ facet must determine the same $r$-facet in $\mathcal{B}(H)$. This follows from Lemma III.7 combined with [[12], 3.1.4]. In particular, the following definition makes sense. When $r=0$, we call an $(r, L)$-facet, an $L$-facet.

Definition III.8. Let $F$ be a $L$-facet, and suppose $x \in F$. Define $H_{F}:=H_{x}$.

Using the Moy-Prasad filtration subgroups, we can show a similar compatibility on the level of groups. More precisely, when $x$ lies in $\mathcal{B}(H)$, we show (in Appendix A)

$$
G_{x}^{+} \cap H=H_{x}^{+}
$$

Moreover, using this fact, it is not difficult to see that 


$$
\left(\mathrm{G}_{x}^{L}\right)^{\circ}=\mathrm{H}_{x}
$$

where $\mathrm{G}_{x}$ and $\mathrm{H}_{x}$ are the connected, reductive $\mathrm{f}$-groups associated to $x$. For these details, see Appendix A.1.

Proposition III.9. Let $F_{i}(i=1,2)$ be nonempty $(r, L)$-facets in some apartment $\mathcal{A} \subset$ $\mathcal{B}(H)$, and suppose $F_{i}^{\prime} \supset F_{i}$ is an r-facet in some apartment $\mathcal{A}^{\prime} \subset \mathcal{B}(G)$ containing $\mathcal{A}$. Define $\mathfrak{g}_{F_{i}}^{\sigma}:=\mathfrak{g}^{\sigma} \cap \mathfrak{g}_{F_{i}}$ and $\mathfrak{g}_{F_{i}}^{\sigma,+}:=\mathfrak{g}^{\sigma} \cap \mathfrak{g}_{F_{i}}^{+}$. Then, for $i=1,2$, we have:

1. $\mathfrak{g}_{F_{i}}=\mathfrak{h}_{F_{i}} \oplus \bigoplus_{\sigma} \mathfrak{g}_{F_{i}}^{\sigma}$ and $\mathfrak{g}_{F_{i}}^{+}=\mathfrak{h}_{F_{i}}^{+} \oplus \bigoplus_{\sigma} \mathfrak{g}_{F_{i}}^{\sigma,+}$

2. $F_{1}^{\prime}=F_{2}^{\prime}$ if and only if $F_{1}=F_{2}$

Proof. Since each $l \in L$ is an automorphism of $\mathbf{G}$ defined over $k$, the map $l$ induces an action on $\mathcal{B}(G)$ which is compatible with all structures on $\mathcal{B}(G)$. In particular, by [[2], Proposition 2.2.1], since $F_{i} \subset \mathcal{B}(H)$, we have that $\mathfrak{g}_{F_{i}}$ and $\mathfrak{g}_{F_{i}}^{+}$are $L$-stable. Let $p_{0}$ denote the projection $\frac{1}{|L|} \sum_{l \in L} l$ from $\mathfrak{g}$ onto $\mathfrak{h}$, and let $p_{\sigma}$ denote the projection $\frac{\operatorname{dim} V^{\sigma}}{|L|} \sum_{l \in L} \chi_{V^{\sigma}}\left(l^{-1}\right) l$ onto $\mathfrak{g}^{\sigma}$, where $\left(\sigma, V^{\sigma}\right.$ is an irreducible sub-representation of $\mathfrak{g}^{\sigma}$, and $\chi_{V^{\sigma}}$ is the character associated to $V^{\sigma}$. Let $X \in \mathfrak{g}_{F_{i}}=\mathfrak{g}_{x, r}$. Write

$$
X=p_{0}(X)+\sum_{\sigma} p_{\sigma}(X) \in\left(\mathfrak{h} \cap \mathfrak{g}_{F_{i}}\right) \oplus \bigoplus_{\sigma} \mathfrak{g}_{F_{i}}^{\sigma}
$$

Since $\mathfrak{g}_{x, r}$ and $\mathfrak{g}_{x, r^{+}}$are $L$-stable, and since $\mathfrak{h}, \mathfrak{g}^{\sigma}$ and $\mathfrak{g}_{x, r}$ and $\mathfrak{g}_{x, r^{+}}$are all $R$-modules, and $|L|$ is a unit, by Lemma III.7, the above line simplifies to

$$
\mathfrak{g}_{x, r}=\left(\mathfrak{h} \cap \mathfrak{g}_{x, r}\right) \oplus \bigoplus_{\sigma} \mathfrak{g}_{x, r}^{\sigma}=\mathfrak{h}_{F_{i}} \oplus \bigoplus_{\sigma} \mathfrak{g}_{F_{i}}^{\sigma}
$$

and

$$
\mathfrak{g}_{x, r^{+}}=\left(\mathfrak{h} \cap \mathfrak{g}_{x, r^{+}}\right) \oplus \bigoplus_{\sigma} \mathfrak{g}_{x, r^{+}}^{\sigma}=\mathfrak{h}_{F_{i}}^{+} \oplus \bigoplus_{\sigma} \mathfrak{g}_{F_{i}}^{\sigma,+}
$$


For the second claim, the forward implication is trivial. For the other direction, note that if $F_{1}=F_{2}$, then

$$
\mathfrak{g}_{F_{1}^{\prime}}=\mathfrak{g}_{F_{1}}=\mathfrak{g}_{F_{2}}=\mathfrak{g}_{F_{2}^{\prime}}
$$

and

$$
\mathfrak{g}_{F_{1}^{\prime}}^{+}=\mathfrak{g}_{F_{1}}^{+}=\mathfrak{g}_{F_{2}}^{+}=\mathfrak{g}_{F_{2}^{\prime}}^{+}
$$

which is true if and only if $F_{1}^{\prime}=F_{2}^{\prime}$ by [[12], Lemma 3.1.4].

\subsection{Generalized $(r, L)$-facets}

For the following definition, recall (from [[12], 3.2.1]) that for $x \in \mathcal{B}(G)$, the set $F^{*}(x)=\left\{y \in \mathcal{B}(G) \mid \mathfrak{g}_{x, r}=\mathfrak{g}_{y, r}\right.$ and $\left.\mathfrak{g}_{x, r^{+}}=\mathfrak{g}_{y, r^{+}}\right\}$is called a generalized $r$-facet.

Definition III.10. Let $x \in \mathcal{B}(H)$. Define

$$
F_{L}^{*}(x):=F^{*}(x)^{L}
$$

\section{Definition III.11.}

$$
\mathcal{F}_{L}(r):=\left\{F_{L}^{*}(x) \mid x \in \mathcal{B}(H)\right\}
$$

We call an element of $\mathcal{F}_{L}(r)$ a generalized $(r, L)$-facet.

Remark III.12. We briefly mention a fact which will be used many times throughout this section. By [[5], 4.6.28], if $\mathcal{A}, \tilde{\mathcal{A}}$ are two apartments in $\mathcal{B}(H)$ such that $\Omega=$ $\{x, y\} \subset \mathcal{A} \cap \tilde{\mathcal{A}}$, then there exists an element $h \in H_{x} \cap H_{y}$ such that $h \mathcal{A}=\tilde{\mathcal{A}}$. More succinctly stated, $H_{\Omega}$ acts transitively on the apartments of $\mathcal{B}(H)$ containing $\Omega$.

Remark III.13. We remark that if $F_{L}^{*}(x)$ is a generalized $(r, L)$-facet, and $\mathcal{A}$ is an apartment of $\mathcal{B}(H)$ such that $F_{L}^{*}(x) \cap \mathcal{A} \neq \emptyset$, then $F_{L}^{*}(x) \cap \mathcal{A}$ is an $(r, L)$-facet of $\mathcal{A}$. 
Lemma III.14. Let $x \in \mathcal{B}(H)$ and $\mathcal{A}$ an apartment in $\mathcal{B}(H)$ such that $F:=F_{L}^{*}(x) \cap$ $\mathcal{A} \neq \emptyset$. For all $y \in F$, we have

$$
F_{L}^{*}(x)=H_{y} \cdot F
$$

Proof. Let $y \in F$.

" $\subset$ ": Let $z \in F_{L}^{*}(x)$, and let $\tilde{A}$ be an apartment in $\mathcal{B}(H)$ containing $y$ and $z$. By Remark III.12, there exists an element $h \in H_{y}$ such that $h z \in \mathcal{A}$. We have

$$
\mathfrak{g}_{h z, r}={ }^{h} \mathfrak{g}_{z, r}={ }^{h} \mathfrak{g}_{x, r}={ }^{h} \mathfrak{g}_{y, r}=\mathfrak{g}_{h y, r}=\mathfrak{g}_{y, r}=\mathfrak{g}_{x, r}
$$

and similarly,

$$
\mathfrak{g}_{h z, r^{+}}={ }^{h} \mathfrak{g}_{z, r^{+}}={ }^{h} \mathfrak{g}_{x, r^{+}}={ }^{h} \mathfrak{g}_{y, r^{+}}=\mathfrak{g}_{h y, r^{+}}=\mathfrak{g}_{y, r^{+}}=\mathfrak{g}_{x, r^{+}}
$$

Thus, $h z \in \mathcal{A} \cap F_{L}^{*}(x)=F$, so, in particular, $z \in H_{y} \cdot F$.

" $\supset$ ": Let $z \in F$ and $h \in H_{y}$. Then

$$
\mathfrak{g}_{h z, r}={ }^{h} \mathfrak{g}_{z, r}={ }^{h} \mathfrak{g}_{y, r}=\mathfrak{g}_{h y, r}=\mathfrak{g}_{y, r}=\mathfrak{g}_{x, r}
$$

and

$$
\mathfrak{g}_{h z, r^{+}}={ }^{h} \mathfrak{g}_{z, r^{+}}={ }^{h} \mathfrak{g}_{y, r^{+}}=\mathfrak{g}_{h y, r^{+}}=\mathfrak{g}_{y, r^{+}}=\mathfrak{g}_{x, r^{+}}
$$

Thus, $h z \in F_{L}^{*}(x)$.

Corollary III.15. If $F_{L}^{*} \in \mathcal{F}_{L}(r)$, then the image of $F_{L}^{*}$ in the reduced building of $H$ is bounded.

Proof. Let $x \in F_{L}^{*}$, and let $F^{*}$ be the generalized $r$-facet in $\mathcal{B}(G)$ containing $x$. The result follows directly from the result in [[12], Corollary 3.2.7] since the image of $F_{L}^{*}$ 
is contained in the image of $F^{*}$ in the reduced building.

Lemma III.16. Let $x \in B(H)$. We have

$$
N_{H}\left(\mathfrak{g}_{x, r}\right) \cap N_{H}\left(\mathfrak{g}_{x, r^{+}}\right)=\operatorname{stab}_{H}\left(F_{L}^{*}(x)\right) .
$$

Proof. " $\supset$ ": If $h \in \operatorname{stab}_{H}\left(F_{L}^{*}(x)\right)$, then $h x \in F_{L}^{*}(x)$, so, in particular,

$$
{ }^{h} \mathfrak{g}_{x, r}=\mathfrak{g}_{h x, r}=\mathfrak{g}_{x, r}
$$

and

$$
{ }^{h} \mathfrak{g}_{x, r^{+}}=\mathfrak{g}_{h x, r^{+}}=\mathfrak{g}_{x, r^{+}} .
$$

" $\subset$ ": Let $n \in N_{H}\left(\mathfrak{g}_{x, r}\right) \cap N_{H}\left(\mathfrak{g}_{x, r^{+}}\right)$. Choose $z \in F_{L}^{*}(x)$. Since $n$ normalizes the lattices $\mathfrak{g}_{x, r}$ and $\mathfrak{g}_{x, r^{+}}$, we have

$$
\mathfrak{g}_{n z, r}={ }^{n} \mathfrak{g}_{z, r}={ }^{n} \mathfrak{g}_{x, r}=\mathfrak{g}_{x, r}
$$

and

$$
\mathfrak{g}_{n z, r^{+}}={ }^{n} \mathfrak{g}_{z, r^{+}}={ }^{n} \mathfrak{g}_{x, r^{+}}=\mathfrak{g}_{x, r^{+}} .
$$

This implies, by definition of $F^{*}(x)$, that $n z \in F^{*}(x)$. Since $z \in \mathcal{B}(H)$, and $n \in H$, we have $n z \in F^{*}(x) \cap \mathcal{B}(H)=F^{*}(x)^{L}=F_{L}^{*}(x)$. Thus, since $z \in F_{L}^{*}(x)$ was arbitrary, $n F_{L}^{*}(x) \subset F_{L}^{*}(x)$ and thus $n \in \operatorname{stab}_{H}\left(F_{L}^{*}(x)\right)$.

Lemma III.17. Let $F_{L}^{*} \in \mathcal{F}_{L}(r)$ and $\mathcal{A}$ an apartment in $B(H)$ such that $F:=$ $F_{L}^{*} \cap \mathcal{A} \neq \emptyset$. Then

$$
\bar{F}=\overline{F_{L}^{*}} \cap \mathcal{A} .
$$


Proof. " $\subset$ ": This inclusion is clear since $\overline{A \cap B} \subset \bar{A} \cap \bar{B}$ for any two subsets $A, B$ of $\mathcal{B}(H)$.

" $\supset$ " : Let $x \in \overline{F_{L}^{*}} \cap \mathcal{A}$. We will produce a sequence converging to $x$ which lies in $F$. Since $x \in \overline{F_{L}^{*}}$, there exists a sequence $\left\{x_{n}\right\}$ in $F_{L}^{*}$ converging to $x$. Fix $y \in F$. Without loss of generality, assume $\operatorname{dist}\left(x_{n}, x\right)<\frac{1}{n}$ for each $n$. Note that $\bigcup_{x \in \bar{C}} \bar{C}$ contains a neighborhood of $x$, where $C$ ranges over all alcoves in $\mathcal{B}(H)$. Thus, for large $n$, there exist alcoves $C_{n} \subset \mathcal{B}(H)$ such that $x_{n}$ and $x$ lie in $\overline{C_{n}}$. Let $\mathcal{A}_{n}$ be an apartment in $\mathcal{B}(H)$ which contains $C_{n}$ and $y$. We now fix $n$. Since $x$ and $y$ lie in $\mathcal{A}_{n} \cap \mathcal{A}$, by Remark III.12, there is an element $h_{n} \in H$ that maps $\mathcal{A}_{n}$ to $\mathcal{A}$ and fixes $x$ and $y$. In particular, since $h_{n} x=x$, we have

$$
\operatorname{dist}\left(h_{n} x_{n}, x\right)=\operatorname{dist}\left(x_{n}, x\right)<\frac{1}{n} .
$$

Now, by Lemma III.16, since $h_{n} y=y$ for each $n$, we have $h_{n} \in N_{H}\left(\mathfrak{g}_{y, r}\right) \cap N_{H}\left(\mathfrak{g}_{y, r^{+}}\right)=$ $\operatorname{stab}_{H}\left(F_{L}^{*}\right)$. Thus, $h_{n} x_{n} \in F_{L}^{*}$ and since $h_{n} \mathcal{A}_{n}=\mathcal{A}$, we also have $h_{n} x_{n} \in \mathcal{A}$, so $h_{n} x_{n} \in F_{L}^{*} \cap \mathcal{A}=F$. Therefore, $\left\{h_{n} x_{n}\right\}$ is our desired sequence.

Definition III.18. For $F_{L}^{*} \in \mathcal{F}_{L}(r)$ and $\delta>0$, define

$$
F_{L}^{*}(\delta):=\left\{x \in F_{L}^{*} \mid \operatorname{dist}(x, z) \geq \delta \text { for all } z \in \overline{F_{L}^{*}} \backslash F_{L}^{*}\right\}
$$

Lemma III.19. Suppose $F_{L}^{*} \in \mathcal{F}_{L}(r)$ and $\delta>0$. Then $F_{L}^{*}(\delta)$ is a convex, closed, $\operatorname{stab}_{H}\left(F_{L}^{*}\right)$-invariant subset of $\mathcal{B}(H)$. Also, $F_{L}^{*}(\delta)$ is nonempty if and only if there exists an apartment $\mathcal{A}$ in $\mathcal{B}(H)$ such that the following subset of $F:=F_{L}^{*} \cap \mathcal{A}$

$$
F_{L, \mathcal{A}}(\delta):=\{x \in F \mid \operatorname{dist}(x, z) \geq \delta \text { for all } z \in \bar{F} \backslash F\}
$$

is nonempty. 
Proof. To see that $F_{L}^{*}(\delta)$ is closed, suppose $\left\{x_{n}\right\} \subset F_{L}^{*}(\delta)$ is a sequence converging to some $x \in \mathcal{B}(H)$. By the triangle inequality, we have

$$
\operatorname{dist}(x, z) \geq \operatorname{dist}\left(x_{n}, z\right)-\operatorname{dist}\left(x_{n}, x\right) \geq \delta-\operatorname{dist}\left(x_{n}, x\right),
$$

for all $z \in \overline{F_{L}^{*}} \backslash F_{L}^{*}$, so taking $n \rightarrow \infty$, we see that $\operatorname{dist}(x, z) \geq \delta$.

For $\operatorname{stab}_{H}\left(F_{L}^{*}\right)$-invariance, we note that any element $h \in \operatorname{stab}_{H}\left(F_{L}^{*}\right)$ sends the boundary of $F_{L}^{*}$ to itself. In particular, for $z \in \bar{F}_{L}^{*} \backslash F_{L}^{*}$, there exists an element $w \in \bar{F}_{L}^{*} \backslash F_{L}^{*}$ such that $h w=z$. Thus, if $x \in F_{L}^{*}(\delta)$, then

$$
(\dagger) \operatorname{dist}(h x, z)=\operatorname{dist}(h x, h w)=\operatorname{dist}(x, w) \geq \delta \text {. }
$$

We now prove the final statement of the lemma.

" $\Rightarrow$ " : If $F_{L}^{*}(\delta)$ is nonempty, then there exists some apartment $\mathcal{A}$ for which $F_{L}^{*}(\delta) \cap \mathcal{A}$, and hence $F_{L, \mathcal{A}}(\delta)$, is nonempty.

" $\Leftarrow "$ : We will prove a stronger claim here that will be used later to prove convexity of $F_{L}^{*}(\delta)$. In particular, we show that if there is an apartment $\mathcal{A} \subset \mathcal{B}(H)$ such that $F_{L, \mathcal{A}}(\delta) \neq \emptyset$, then $H_{y} F_{L, \mathcal{A}}(\delta)=F_{L}^{*}(\delta)$ for all $y \in F_{L, \mathcal{A}}(\delta)$. This will show that $F_{L}^{*}(\delta) \neq$ $\emptyset$ whenever $F_{L, \mathcal{A}}(\delta) \neq \emptyset$. Suppose $\mathcal{A} \subset \mathcal{B}(H)$ is an apartment such that $F_{L, \mathcal{A}}(\delta) \neq \emptyset$, and let $w \in F_{L, \mathcal{A}}(\delta)$. We first show $H_{w} F_{L, \mathcal{A}}(\delta) \subset F_{L}^{*}(\delta)$.

" $\subset$ " : Note that $H_{w} \subset \operatorname{stab}_{H}\left(F_{L}^{*}\right)$ by an application of Lemma III.16. Thus by $(\dagger)$, we have that $F_{L}^{*}(\delta)$ is $H_{w}$-invariant. As a consequence, it suffices to show that $F_{L, \mathcal{A}}(\delta) \subset F_{L}^{*}(\delta)$. Let $x \in F_{L, \mathcal{A}}(\delta)$ and $z \in \overline{F_{L}^{*}} \backslash F_{L}^{*}$. By [[4], 2.3.1], we may choose an apartment $\tilde{\mathcal{A}}$ containing $x$ and $z$. By Remark III.12, there is an element $h \in H_{x}$ that maps $\tilde{\mathcal{A}}$ onto $\mathcal{A}$. Since $h x=x$, by an application of Lemma III.16, $h \in \operatorname{stab}_{H}\left(F_{L}^{*}\right)$. Since $h \tilde{\mathcal{A}}=\mathcal{A}$, we have $h z \in \mathcal{A}$, so in particular, $h z \in\left(\overline{F_{L}^{*}} \backslash F_{L}^{*}\right) \cap \mathcal{A}$. Thus, by Lemma III.17, 


$$
h z \in\left(\overline{F_{L}^{*}} \cap \mathcal{A}\right) \backslash\left(F_{L}^{*} \cap \mathcal{A}\right)=\overline{F_{L, \mathcal{A}}} \backslash F_{L, \mathcal{A}} .
$$

Again, since $h \in H_{x}$, we have $\operatorname{dist}(x, z)=\operatorname{dist}(x, h z) \geq \delta$, so since $z$ was arbitrary, we must have $x \in F_{L}^{*}(\delta)$.

" $\supset "$ : Now, we show that $H_{w} F_{L, \mathcal{A}}(\delta) \supset F_{L}^{*}(\delta)$ for all $w \in F_{L, \mathcal{A}}(\delta)$. Let $x \in F_{L}^{*}(\delta)$. By Lemma III.14, there exist elements $h \in H_{w}$ and $z \in F$ such that $h z=x$. Arguing as usual, since $w \in F_{L}^{*}$, we have $H_{w} \subset \operatorname{stab}_{H}\left(F_{L}^{*}\right)$, so, in particular,

$$
x \in h F \cap F_{L}^{*}(\delta)=h\left(F \cap F_{L}^{*}(\delta)\right) \subset h F_{L, \mathcal{A}}(\delta) .
$$

Lastly, we must show that $F_{L}^{*}(\delta)$ is a convex subset of $\mathcal{B}(H)$. Assume $F_{L}^{*}(\delta)$ is nonempty. We first show that $F_{L, \mathcal{A}}(\delta) \subset \mathcal{A}$ is convex. Choose an origin $O$ in $\mathcal{A}$. Note that the geodesics of $\mathcal{A}$ are nothing more than segments, so if $x, y \in F_{L, \mathcal{A}}(\delta)$ and $z \in[x, y]$, then considering $x, y$, and $z$ as the vectors, $x-O, y-O$, and $z-O$, respectively, we have $z=t x+(1-t) y$ for some $t \in[0,1]$. Thus, for all $z^{\prime} \in \bar{F} \backslash F$,

$$
\begin{aligned}
\operatorname{dist}\left(t x+(1-t) y, z^{\prime}\right) & \geq \operatorname{dist}\left(t x, t z^{\prime}\right)-\operatorname{dist}\left((1-t) y,(1-t) z^{\prime}\right) \\
& =t \operatorname{dist}\left(x, z^{\prime}\right)+(1-t) \operatorname{dist}\left(y, z^{\prime}\right) \geq \delta
\end{aligned}
$$

So $z$ lies in $F_{L, \mathcal{A}}(\delta)$.

Now, suppose $F_{L}^{*}(\delta)$ is nonempty. There is an apartment $\mathcal{A}$ such that $F_{L, \mathcal{A}}(\delta)$ is nonempty. Let $x, y \in F_{L}^{*}(\delta)$ and $z \in F_{L, \mathcal{A}}(\delta)$. Previously in this proof, we showed that for all $w \in F_{L, \mathcal{A}}(\delta)$, we have

$$
(\ddagger) H_{w} F_{L, \mathcal{A}}(\delta)=F_{L}^{*}(\delta)
$$

so there is some $h \in H_{z}$ such that $h x \in F_{L, \mathcal{A}}(\delta)$. Since $h \in H_{z} \subset \operatorname{stab}_{H}\left(F_{L}^{*}\right)$, we have 
$h y \in F_{L}^{*}$, so for all $z^{\prime} \in \overline{F_{L}^{*}} \backslash F_{L}^{*}$, we have

$$
\operatorname{dist}\left(h y, z^{\prime}\right)=\operatorname{dist}\left(h y, h w^{\prime}\right)=\operatorname{dist}\left(y, w^{\prime}\right) \geq \delta
$$

where $w^{\prime} \in \overline{F_{L}^{*}} \backslash F_{L}^{*}$ such that $h w^{\prime}=z$. Thus, $h y \in F_{L}^{*}(\delta)$. Applying ( $\left.\ddagger\right)$ again, there exists some $h^{\prime} \in H_{h x}$ such that $h^{\prime} h y \in F_{L, \mathcal{A}}(\delta)$. Thus, since $F_{L, \mathcal{A}}^{*}(\delta)$ is convex, we have

$$
\left[h^{\prime} h x, h^{\prime} h y\right] \subset F_{L, \mathcal{A}}^{*}(\delta) \subset F_{L}^{*}(\delta)
$$

so, in particular, since $F_{L, \mathcal{A}}^{*}(\delta)$ is $\operatorname{stab}_{H}\left(F_{L}^{*}\right)$-invariant, $[x, y] \subset F_{L}^{*}(\delta)$.

Definition III.20. For $F_{L}^{*} \in \mathcal{F}_{L}(r)$, define

$C\left(F_{L}^{*}\right):=\left\{y \in F_{L}^{*} \mid\right.$ for all apartments $\mathcal{A}$ of $\mathcal{B}(H)$ for which $\mathcal{A} \cap F_{L}^{*} \neq \emptyset$ we have $\left.y \in \mathcal{A}\right\}$.

Remark III.21. Suppose $\mathbf{H}$ is semisimple. Following the discussion in [[31], 2.2.1], there is a map

$$
\pi: H \times \mathcal{B}(H) \rightarrow \mathcal{B}(H) \times \mathcal{B}(H)
$$

given by $(h, x) \mapsto(h x, x)$, with the property that the inverse images of bounded sets are bounded. We note that if $\Omega$ is a bounded subset of $\mathcal{B}(H)$, this tells us that

$$
\operatorname{stab}_{H}(\Omega) \times \Omega=\pi^{-1}(\Omega \times \Omega)
$$

is bounded. In particular, $\operatorname{stab}_{H}(\Omega)$ is bounded whenever $\Omega$ is bounded.

Corollary III.22. If $F_{L}^{*} \in \mathcal{F}_{L}(r)$, then $C\left(F_{L}^{*}\right) \neq \emptyset$.

Proof. Let $\mathrm{pr}_{s s}$ denote the projection from the enlarged building of $H$ to the reduced building of $H$. Since 


$$
C\left(\operatorname{pr}_{s s}\left(F_{L}^{*}\right)\right) \neq \emptyset \Rightarrow C\left(F_{L}^{*}\right) \neq \emptyset,
$$

we may assume $\mathbf{H}$ is semisimple.

By Corollary III.15, $F_{L}^{*}$ is bounded in $\mathcal{B}(H)$, so, by Remark III.21, the stabilizer $N:=\operatorname{stab}_{H}\left(F_{L}^{*}\right)$ is a bounded subgroup of $H$. If $F_{L}^{*}$ consists of a point $x$, then $\mathcal{A} \cap F_{L}^{*}=\tilde{\mathcal{A}} \cap F_{L}^{*}=\{x\}$ for all apartments in $\mathcal{B}(H)$ that meet $\{x\}$, so clearly $x \in C\left(F_{L}^{*}\right)$.

Suppose $F_{L}^{*}$ is not a point, and let $\mathcal{A} \subset \mathcal{B}(H)$ be an apartment such that $F_{L}^{*} \cap \mathcal{A} \neq$ $\emptyset$. Then, by Lemma III.14, since $\operatorname{dim} F_{L}^{*}>0$, we have $\operatorname{dim} F_{L}^{*} \cap \mathcal{A}>0$. As a consequence, there exists some $\delta>0$ for which $F_{L, \mathcal{A}}(\delta)$ is nonempty. By Lemma III.19, this implies $\emptyset \neq F_{L}^{*}(\delta)$ is convex and $N$-stable. Thus, by [[5], 3.2.4], since a bounded group of isometries acting on a nonempty, closed, convex set $F$ of $\mathcal{B}(H)$ has a fixed point, there exists some $y \in F_{L}^{*}(\delta)$ such that $n y=y$ for all $n \in N$. Suppose now that $\tilde{\mathcal{A}}$ is an apartment of $\mathcal{B}(H)$ for which $F_{\tilde{\mathcal{A}}}:=F_{L}^{*} \cap \tilde{\mathcal{A}} \neq \emptyset$. Let $z \in F_{\tilde{\mathcal{A}}}$. By Lemma III.14, we have $H_{z} F_{\tilde{\mathcal{A}}}=F_{L}^{*}$, and by Lemma III.16, we have $H_{z} \subset N$. In particular, $h y=y$ for all $h \in H_{z}$. Therefore, $y \in F_{\tilde{\mathcal{A}}} \subset \tilde{\mathcal{A}}$, so $y \in C\left(F_{L}^{*}\right)$.

Corollary III.23. If $\mathcal{A}_{1}$ and $\mathcal{A}_{2}$ are two apartments in $\mathcal{B}(H)$, and $F_{L}^{*} \in \mathcal{F}_{L}(r)$ such that $F_{L}^{*} \cap \mathcal{A}_{i} \neq \emptyset$, then $\operatorname{dim} A\left(F_{L}^{*} \cap \mathcal{A}_{1}, \mathcal{A}_{1}\right)=\operatorname{dim} A\left(F_{L}^{*} \cap \mathcal{A}_{2}, \mathcal{A}_{2}\right)$.

Definition III.24. Let $F_{L}^{*} \in \mathcal{F}_{L}(r)$. Let $\mathcal{A}$ be an apartment in $\mathcal{B}(H)$ with $F_{L}^{*} \cap \mathcal{A} \neq \emptyset$. Define

$$
\operatorname{dim} F_{L}^{*}:=\operatorname{dim} A\left(F_{L}^{*} \cap \mathcal{A}, \mathcal{A}\right) .
$$

Definition III.25. Suppose $F_{L}^{*} \in \mathcal{F}_{L}(r)$. Fix $x \in F_{L}^{*}$ and define

$$
\mathfrak{g}_{F_{L}^{*}}:=\mathfrak{g}_{x, r}
$$

and 


$$
\mathfrak{g}_{F_{L}^{*}}^{+}:=\mathfrak{g}_{x, r^{+}}
$$

Let $F_{L}^{*}$ be a generalized $(r, L)$-facet. Recall that the quotient $V:=\mathfrak{g}_{F_{L}^{*}} / \mathfrak{g}_{F_{L}^{*}}^{+}$is an $\mathfrak{f}$-vector space. Moreover, by Proposition III.9, both $\mathfrak{g}_{F_{L}^{*}}$ and $\mathfrak{g}_{F_{L}^{*}}^{+}$are $L$-stable, so $V$ is equipped with automorphisms induced by elements of $L$. The following lemma is an analogue of Proposition III.9 when thinking of the quotient $\mathfrak{g}_{F_{L}^{*}} / \mathfrak{g}_{F_{L}^{*}}^{+}$as a representation of $L$.

Lemma III.26. Suppose $F_{L}^{*}$ is a generalized $(r, L)$-facet of $\mathcal{B}(H)$. Then

$$
\mathfrak{g}_{F_{L}^{*}} / \mathfrak{g}_{F_{L}^{*}}^{+}=\mathfrak{h}_{F_{L}^{*}} / \mathfrak{h}_{F_{L}^{*}}^{+} \oplus \bigoplus_{\sigma} \mathfrak{g}_{F_{L}^{*}}^{\sigma} / \mathfrak{g}_{F_{L}^{*}}^{\sigma,+}
$$

where we use Lemma III.7 to identify $\mathfrak{h}_{F_{L}^{*}} / \mathfrak{h}_{F_{L}^{*}}^{+}$and $\mathfrak{g}_{F_{L}^{*}}^{\sigma} / \mathfrak{g}_{F_{L}^{*}}^{\sigma,+}$ with their images inside $\mathfrak{g}_{F_{L}^{*}} / \mathfrak{g}_{F_{L}^{*}}^{+}$

Proof. We first show that $\mathfrak{h}_{F_{L}^{*}} / \mathfrak{h}_{F_{L}^{*}}^{+}$is the $L$-fixed subspace of $\mathfrak{g}_{F_{L}^{*}} / \mathfrak{g}_{F_{L}^{*}}^{+}$. By Lemma III.7, we have $\mathfrak{h}_{x, r}=\mathfrak{g}_{x, r} \cap \mathfrak{h}$. Thus, we can identify $\mathfrak{h}_{F_{L}^{*}} / \mathfrak{h}_{F_{L}^{*}}^{+}$as a subset of $\mathfrak{g}_{F_{L}^{*}} / \mathfrak{g}_{F_{L}^{*}}^{+}$. Moreover, it is clear that $L$ fixes every element of $\mathfrak{h}_{F_{L}^{*}} / \mathfrak{h}_{F_{L}^{*}}^{+}$. Thus, $\mathfrak{h}_{F_{L}^{*}} / \mathfrak{h}_{F_{L}^{*}}^{+} \subset\left(\mathfrak{g}_{F_{L}^{*}} / \mathfrak{g}_{F_{L}^{*}}^{+}\right)^{L}$.

Now, let $\bar{X} \in\left(\mathfrak{g}_{F_{L}^{*}} / \mathfrak{g}_{F_{L}^{*}}^{+}\right)^{L}$, and let $X=X_{0}+\sum_{\sigma} X_{\sigma}$ be a representative of $\bar{X}$ in $\mathfrak{g}_{F_{L}^{*}}$. Let $p_{0}: \mathfrak{g} \rightarrow \mathfrak{h}$ denote the projection onto the isotypic component corresponding to the trivial representation. Then,

$$
p_{0}(X)-X \in \mathfrak{g}_{F_{L}^{*}}^{+}
$$

so since $p_{0}$ fixes $X_{0}$, we must have

$$
-\sum_{\sigma \neq 1} X_{\sigma} \in \mathfrak{g}_{F_{L}^{*}}^{+}
$$

so, by Proposition III.9, each $X_{\sigma}$ lies in $\mathfrak{g}_{F_{L}^{*}}^{+}$. In particular, we have 


$$
X_{0}+\mathfrak{h}_{F_{L}^{*}}^{+} \mapsto X+\mathfrak{g}_{F_{L}^{*}}^{+}
$$

so $\mathfrak{h}_{F_{L}^{*}} / \mathfrak{h}_{F_{L}^{*}}^{+}=\left(\mathfrak{g}_{F_{L}^{*}} / \mathfrak{g}_{F_{L}^{*}}^{+}\right)^{L}$.

Now, let $\bar{X} \in\left(\mathfrak{g}_{F_{L}^{*}} / \mathfrak{g}_{F_{L}^{*}}^{+}\right)^{\sigma}$, and let $X=X_{0}+\sum_{\tau} X_{\tau}$ be a representative in $\mathfrak{g}_{F_{L}^{*}}$. Then, letting $p_{\sigma}$ denote the projection operator from $\mathfrak{g}$ to $\mathfrak{g}^{\sigma}$ described in the proof of Proposition III.9, we have

$$
p_{\sigma}(X)-X \in \mathfrak{g}_{F_{L}^{*}}^{+},
$$

so since $p_{\sigma}$ fixes $X_{\sigma}$, we have

$$
-X_{0}-\sum_{\tau \neq \sigma} X_{\tau} \in \mathfrak{g}_{F_{L}^{*}}^{+}
$$

In particular, $X_{\sigma}+\mathfrak{g}_{F_{L}^{*}}^{\sigma,} \mapsto X+\mathfrak{g}_{F_{L}^{*}}^{+}$, so $\mathfrak{g}_{F_{L}^{*}}^{\sigma} / \mathfrak{g}_{F_{L}^{*}}^{\sigma,+}=\left(\mathfrak{g}_{F_{L}^{*}} / \mathfrak{g}_{F_{L}^{*}}^{+}\right)^{\sigma}$.

Definition III.27. Suppose $F_{L}^{*} \in \mathcal{F}_{L}(r)$, and $\mathcal{A}$ is an apartment in $\mathcal{B}(H)$. Define

$$
A\left(\mathcal{A}, F_{L}^{*}\right):=A\left(F_{L}^{*} \cap \mathcal{A}, \mathcal{A}\right)
$$

\subsection{Standard lifts and $r$-associativity}

Remark III.28. Let $x \in \mathcal{B}(H)$, and let $F_{L}^{*}(x) \in \mathcal{F}_{L}(r)$. We call a generalized $r$-facet $F^{*}$ in $\mathcal{B}(G)$ the standard lift of $F_{L}^{*}(x)$ if $F^{*}$ is the generalized $r$-facet in $\mathcal{B}(G)$ containing $x$, as defined in [[12], 3.2.1] and in Section III.2.

Lemma III.29. Let $y \in \mathcal{B}(G)$. The generalized $r$-facet $F^{*}(y)$ is L-stable if and only if $F^{*}(y) \cap \mathcal{B}(H) \neq \emptyset$. In particular, if $F_{L}^{*}(x) \in \mathcal{F}_{L}(r)$, then the standard lift $F^{*}(x)$ of $F_{L}^{*}(x)$ is L-stable. 
Proof. " $\Leftarrow$ ": Let $z \in F^{*}(x)$ with $x \in \mathcal{B}(H)$. We must verify that $l(z) \in F^{*}(x)$, for all $l \in L$. This occurs if and only if

$$
\text { (†) } \mathfrak{g}_{l(z), r}=\mathfrak{g}_{x, r} \text { and } \mathfrak{g}_{l(z), r^{+}}=\mathfrak{g}_{x, r^{+}} \text {for all } l \in L \text {. }
$$

Since $x$ lies in $\mathcal{B}(H)$, we have $l^{-1}(x)=x$, so $z \in F^{*}\left(l^{-1}(x)\right)$. Thus, we have

$$
\mathfrak{g}_{z, r}=\mathfrak{g}_{l^{-1}(x), r} \text { and } \mathfrak{g}_{z, r^{+}}=\mathfrak{g}_{l^{-1}(x), r^{+}}
$$

which, after applying $l$ to the left and right-hand sides, is equivalent to $(\dagger)$.

" $\Rightarrow$ ": Let $F^{*}=F^{*}(y)$, for some $y \in \mathcal{B}(G)$, and let $l \in L$. By [[12], Lemma 3.2.11], $F^{*}(\delta)$ is a convex, closed, $\operatorname{stab}_{G}\left(F^{*}\right)$-invariant set of $\mathcal{B}(G)$. Also, note that $l$ preserves the boundary of $F^{*}$, and $l$ acts on $\mathcal{B}(G)$ by an isometry. Thus, if $z \in \overline{F^{*}} \backslash F^{*}$, and $x \in F^{*}(\delta)$, we have

$$
\operatorname{dist}(l(x), z)=\operatorname{dist}\left(l(x), l\left(z^{\prime}\right)\right)=\operatorname{dist}\left(x, z^{\prime}\right) \geq \delta,
$$

for all $z^{\prime} \in \overline{F^{*}} \backslash F^{*}$. In particular, since $l$ was arbitrary, $F^{*}(\delta)$ is $L$-stable. Now, since $L$ is a finite group of isometries, we apply $[[31], 2.3 .1]$ to conclude that $L$ has a fixed point in $F^{*}(\delta)$, and hence in $F^{*}$.

The following proposition gives us a way to translate the work done in Sections III.1 and III.2 into the framework of $L$-stable $r$-facets.

Proposition III.30. Let $F_{1, L}^{*}, F_{2, L}^{*} \in \mathcal{F}_{L}(r)$, and let $\mathcal{A} \subset \mathcal{A}^{\prime}$ be apartments in $\mathcal{B}(H)$ and $\mathcal{B}(G)$, respectively, such that $F_{i, L}^{*} \cap \mathcal{A} \neq \emptyset$, for $i=1,2$. If $F_{1}^{*}$ and $F_{2}^{*}$ are the standard lifts of $F_{1, L}^{*}$ and $F_{2, L}^{*}$, respectively, then

$$
A\left(\mathcal{A}, F_{1, L}^{*}\right)=A\left(\mathcal{A}, F_{2, L}^{*}\right) \Leftrightarrow A\left(\mathcal{A}^{\prime}, F_{1}^{*}\right)=A\left(\mathcal{A}^{\prime}, F_{2}^{*}\right) .
$$

Proof. " $\Leftarrow$ " : We first claim that $A\left(\mathcal{A}^{\prime}, F_{1}^{*}\right)^{L}=A\left(\mathcal{A}, F_{1, L}^{*}\right)$. If this is not true, then, 
since $A\left(\mathcal{A}^{\prime}, F_{1}^{*}\right)^{L}$ is convex, there is an $(r, L)$-facet $C$ in $A\left(\mathcal{A}^{\prime}, F_{1}^{*}\right)^{L}$ properly containing $F_{1, L}^{*} \cap \mathcal{A}$ in its closure. Let $C^{\prime}$ be the $r$-facet in $\mathcal{A}^{\prime}$ containing $C$. Since $F_{1, L}^{*} \cap \mathcal{A} \subset \bar{C}$, we have $F_{1}^{*} \cap \mathcal{A}^{\prime} \subset \overline{C^{\prime}}$. Note that $\overline{C^{\prime}}$ is the union of $C^{\prime}$ and $r$-facets of strictly smaller dimension. Thus, if $\operatorname{dim} F_{1}^{\prime}=\operatorname{dim} C^{\prime}$, then we must have $F_{1}^{\prime}=C^{\prime}$. By our choice of $C^{\prime}, \operatorname{dim} F_{1}^{\prime} \neq \operatorname{dim} C^{\prime}$. In particular, $C^{\prime}$ is a $L$-stable $r$-facet (contained in $A\left(\mathcal{A}^{\prime}, F_{1}^{*}\right)$ by Remark III.1) of strictly larger dimension than $F_{1}^{*} \cap \mathcal{A}^{\prime}$, a contradiction.

" $\Rightarrow$ ": Since $A\left(\mathcal{A}, F_{1, L}^{*}\right)=A\left(\mathcal{A}, F_{2, L}^{*}\right)$, in particular, we know $F_{1}^{*} \cap \mathcal{A}^{\prime}$ intersects $A\left(\mathcal{A}^{\prime}, F_{2}^{*}\right)$ nontrivially. Thus, by Remark III.1, $F_{1}^{*} \cap \mathcal{A}^{\prime}$ is entirely contained in $A\left(\mathcal{A}^{\prime}, F_{2}^{*}\right)$. Similarly, $F_{2}^{*} \cap \mathcal{A}^{\prime}$ is entirely contained inside $A\left(\mathcal{A}^{\prime}, F_{1}^{*}\right)$.

Definition III.31. Let $F_{1, L}^{*}, F_{2, L}^{*} \in \mathcal{F}_{L}(r)$. We say that $F_{1, L}^{*}$ and $F_{2, L}^{*}$ are strongly $r$-associated if for all apartments $\mathcal{A}$ in $\mathcal{B}(H)$ such that $F_{1, L}^{*} \cap \mathcal{A}$ and $F_{2, L}^{*} \cap \mathcal{A}$ are nonempty, we have

$$
A\left(\mathcal{A}, F_{1, L}^{*}\right)=A\left(\mathcal{A}, F_{2, L}^{*}\right)
$$

Remark III.32. By [[12], Lemma 3.3.3] and Proposition III.30, this is the same as demanding that the standard lifts $F_{1}^{*}, F_{2}^{*}$ be strongly $r$-associated in the sense of [[12], Definition 3.3.2].

Lemma III.33. Two generalized $(r, L)$-facets $F_{1, L}^{*}, F_{2, L}^{*} \in \mathcal{F}_{L}(r)$ are strongly $r$ associated if and only if there exists an apartment $\mathcal{A} \subset \mathcal{B}(H)$ for which $F_{1, L}^{*} \cap \mathcal{A}, F_{2, L}^{*} \cap$ $\mathcal{A}$ are nonempty, and

$$
A\left(\mathcal{A}, F_{1, L}^{*}\right)=A\left(\mathcal{A}, F_{2, L}^{*}\right)
$$

Proof. " $\Rightarrow "$ : The definition of strong $r$-associativity proves the forward implication. $" \Leftarrow "$ : Let $\tilde{\mathcal{A}}$ be an apartment for which $A\left(\tilde{\mathcal{A}}, F_{1, L}^{*}\right)=A\left(\tilde{\mathcal{A}}, F_{2, L}^{*}\right) \neq \emptyset$. By $[[12]$, 
Lemma 3.3.3] and Proposition III.30, we have that the standard lifts $F_{1}^{*}$ and $F_{2}^{*}$ (of $F_{1, L}^{*}$ and $F_{2, L}^{*}$ respectively) are strongly $r$-associated. By Proposition III.30, the result follows by taking $L$-fixed points.

Definition III.34. Two generalized $(r, L)$-facets $F_{1, L}^{*}, F_{2, L}^{*} \in \mathcal{F}_{L}(r)$ are said to be $r$ associated if there exists an $h \in H$ such that $F_{1, L}^{*}$ and $h F_{2, L}^{*}$ are strongly $r$-associated.

Lemma III.35. $r$-associativity is an equivalence relation on $\mathcal{F}_{L}(r)$. Whenever two generalized $(r, L)$-facets $F_{1, L}^{*}, F_{2, L}^{*}$ are r-associated, we write $F_{1, L}^{*} \sim F_{2, L}^{*}$.

Proof. For reflexivity, let $F_{L}^{*} \in \mathcal{F}_{L}(r)$. Suppose $\mathcal{A} \subset \mathcal{B}(H)$ such that $\mathcal{A} \cap F_{L}^{*} \neq \emptyset$. We have $A\left(\mathcal{A}, F_{L}^{*}\right)=A\left(\mathcal{A}, F_{L}^{*}\right)$, so the relation is reflexive.

Now, suppose $F_{1, L}^{*} \sim F_{2, L}^{*}$. Then, there exists an apartment $\mathcal{A} \subset \mathcal{B}(H)$ and an element $h \in H$ such that

$$
A\left(\mathcal{A}, F_{1, L}^{*}\right)=A\left(\mathcal{A}, h F_{2, L}^{*}\right) \neq \emptyset
$$

Recall $h A\left(\mathcal{A}, F_{L}^{*}\right)=A\left(h \mathcal{A}, h F_{L}^{*}\right)$ for any $h \in H$. Thus, multiplying the equation above by $h^{-1}$, we obtain

$$
A\left(h^{-1} \mathcal{A}, h^{-1} F_{1, L}^{*}\right)=A\left(h^{-1} \mathcal{A}, F_{2, L}^{*}\right) \neq \emptyset
$$

In particular, $F_{2, L}^{*} \sim F_{1, L}^{*}$

Now, suppose $F_{1, L}^{*}, F_{2, L}^{*}$, and $F_{3, L}^{*}$ are generalized $(r, L)$-facets such that $F_{1, L}^{*} \sim$ $F_{2, L}^{*}$ and $F_{2, L}^{*} \sim F_{3, L}^{*}$. Then, by definition, there exist $h_{2}, h_{3} \in H$ and apartments $\mathcal{A}_{12}, \mathcal{A}_{23} \subset \mathcal{B}(H)$ such that

$$
A\left(\mathcal{A}_{12}, F_{1, L}^{*}\right)=A\left(\mathcal{A}_{12}, h_{2} F_{2, L}^{*}\right) \neq \emptyset
$$


and

$$
A\left(\mathcal{A}_{23}, F_{2, L}^{*}\right)=A\left(\mathcal{A}_{23}, h_{3} F_{3, L}^{*}\right) \neq \emptyset \text {. }
$$

Let $z \in C\left(F_{2, L}^{*}\right)$. Then, since $h_{2}^{-1} \mathcal{A}_{12} \cap F_{2, L}^{*} \neq \emptyset$ and $\mathcal{A}_{23} \cap F_{2, L}^{*} \neq \emptyset$, z lies in $h_{2}^{-1} \mathcal{A}_{12} \cap \mathcal{A}_{23}$. By Remark III.12, there exists some $h \in H_{z} \subset \operatorname{stab}_{H}\left(F_{2, L}^{*}\right)$ (since $h$ fixes $z$ ) such that $h h_{2}^{-1} \mathcal{A}_{12}=\mathcal{A}_{23}$.

Using these facts, we have

$$
\begin{aligned}
\emptyset \neq A\left(\mathcal{A}_{12}, F_{1, L}^{*}\right) & =A\left(\mathcal{A}_{12}, h_{2} F_{2, L}^{*}\right)=h_{2} A\left(h_{2}^{-1} \mathcal{A}_{12}, F_{2, L}^{*}\right) \\
& =h_{2} h^{-1} A\left(\mathcal{A}_{23}, h F_{2, L}^{*}\right)=h_{2} h^{-1} A\left(\mathcal{A}_{23}, F_{2, L}^{*}\right) \\
& =A\left(\mathcal{A}_{12}, h_{2} h^{-1} h_{3} F_{3, L}^{*}\right) .
\end{aligned}
$$

\subsection{Identification of some $\mathfrak{f}$-vector spaces}

Definition III.36. As in [[12], 3.4.1], for $x \in \mathcal{B}(G)$, let $V_{x, r}$ denote the $\mathfrak{f}$-vector space $\mathfrak{g}_{x, r} / \mathfrak{g}_{x, r^{+}}$.

Definition III.37. For $x \in \mathcal{B}(G)$, let $V_{x, r}^{\sigma}$ denote the $\sigma$-isotypic component of $V_{x, r}$ as an $L$-representation.

Remark III.38. We remark that $V_{x, r}^{L}$ (resp. $V_{x, r}^{\sigma}$ ) is identified with the f-vector space $\mathfrak{h}_{x, r} / \mathfrak{h}_{x, r^{+}}\left(\right.$resp. $\left.\mathfrak{g}_{x, r}^{\sigma} / \mathfrak{g}_{x, r^{+}}^{\sigma}\right)$ via Lemma III.26.

Definition III.39. If $F_{L}^{*} \in \mathcal{F}_{L}(r)$ and $x \in F_{L}^{*}$, define $V_{F_{L}^{*}}:=V_{x, r}$ and $V_{F_{L}^{*}}^{\sigma}=V_{x, r}^{\sigma}$.

By Remark III.38, we interpret the quotient maps given by $\mathfrak{h}_{x, r} \rightarrow V_{x, r}^{L}$ and $\mathfrak{g}_{x, r}^{\sigma} \rightarrow$ $V_{x, r}^{\sigma}$. below using this identification. 
Lemma III.40. Suppose $F_{1, L}^{*}, F_{2, L}^{*} \in \mathcal{F}_{L}(r)$ are strongly $r$-associated. Then the natural maps

$$
\begin{gathered}
\mathfrak{h}_{F_{1, L}^{*}} \cap \mathfrak{h}_{F_{2, L}^{*}} \rightarrow V_{F_{i, L}^{*}}^{L} \\
\mathfrak{g}_{F_{1, L}^{*}}^{\sigma} \cap \mathfrak{g}_{F_{2, L}^{*}}^{\sigma} \rightarrow V_{F_{i, L}^{*}}^{\sigma}
\end{gathered}
$$

are surjective with kernels $\mathfrak{h}_{F_{1, L}^{*}}^{+} \cap \mathfrak{h}_{F_{2, L}^{*}}=\mathfrak{h}_{F_{1, L}^{*}} \cap \mathfrak{h}_{F_{2, L}^{*}}^{+}=\mathfrak{h}_{F_{1, L}^{*}}^{+} \cap \mathfrak{h}_{F_{2, L}^{*}}^{+}$and $\mathfrak{g}_{F_{1, L}^{*}}^{\sigma,} \cap \mathfrak{g}_{F_{2, L}^{*}}^{\sigma}=$ $\mathfrak{g}_{F_{1, L}^{*}}^{\sigma} \cap \mathfrak{g}_{F_{2, L}^{*}}^{\sigma,+}=\mathfrak{g}_{F_{1, L}^{*}}^{\sigma,+} \cap \mathfrak{g}_{F_{2, L}^{*}}^{\sigma,+}$, respectively.

Proof. By Remark III.32, we know that the standard lifts $F_{1}^{*}$ and $F_{2}^{*}$ are strongly $r$-associated. Let $e \in V_{F_{i, L}^{*}}^{L}$. By [[12], 3.5.1], there is a lift $X \in \mathfrak{g}_{F_{1, L}^{*}} \cap \mathfrak{g}_{F_{2, L}^{*}}$. Let $X_{0}$ denote the image under the projection of $X$ to $\mathfrak{h}_{F_{i, L}^{*}}$. Then, by the proof of Lemma III.26, $X_{0}$ is mapped to $e$. By Lemma III.7, $X_{0}$ lies in $\mathfrak{h} \cap \mathfrak{g}_{F_{1, L}^{*}} \cap \mathfrak{g}_{F_{2, L}^{*}}=\mathfrak{h}_{F_{1, L}^{*}} \cap \mathfrak{h}_{F_{2, L}^{*}}$. Thus, the map is surjective. If $X$ lies in the kernel of the first map, then, by [[12], 3.5.1], $X$ is contained in $\mathfrak{g}_{F_{1, L}^{*}}^{+} \cap \mathfrak{g}_{F_{2, L}^{*}}=\mathfrak{g}_{F_{1, L}^{*}} \cap \mathfrak{g}_{F_{2, L}^{*}}^{+}=\mathfrak{g}_{F_{1, L}^{*}}^{+} \cap \mathfrak{g}_{F_{2, L}^{*}}^{+}$. Thus, the kernel is $\mathfrak{h} \cap \mathfrak{g}_{F_{1, L}^{*}}^{+} \cap \mathfrak{g}_{F_{2, L}^{*}}=\mathfrak{h}_{F_{1, L}^{*}} \cap \mathfrak{h}_{F_{2, L}^{*}}^{+}=\mathfrak{h}_{F_{1, L}^{*}}^{+} \cap \mathfrak{h}_{F_{2, L}^{*}}^{+}$.

Similarly, let $X \in \mathfrak{g}_{F_{1, L}^{*}}^{\sigma} \cap \mathfrak{g}_{F_{2, L}^{*}}^{\sigma}$, and suppose $X$ is mapped to the trivial coset in $V_{F_{i, L}^{*}}^{\sigma}$. Then, again by [[12], 3.5.1], we have $X \in \mathfrak{g}_{F_{1, L}^{*}}^{+} \cap \mathfrak{g}_{F_{2, L}^{*}}=\mathfrak{g}_{F_{1, L}^{*}} \cap \mathfrak{g}_{F_{2, L}^{*}}^{+}=$ $\mathfrak{g}_{F_{1, L}^{*}}^{+} \cap \mathfrak{g}_{F_{2, L}^{*}}^{+}$, so since $\mathfrak{g}^{\sigma} \cap \mathfrak{g}_{F_{i, L}^{*}}^{+}=\mathfrak{g}_{F_{1, L}^{*}}^{\sigma,+},($ for $i=1,2)$ by definition, we have $X \in$ $\mathfrak{g}_{F_{1, L}^{*}}^{\sigma,+} \cap \mathfrak{g}_{F_{2, L}^{*}}^{\sigma}=\mathfrak{g}_{F_{1, L}^{*}}^{\sigma} \cap \mathfrak{g}_{F_{2, L}^{*}}^{\sigma,+}=\mathfrak{g}_{F_{1, L}^{*}}^{\sigma,+} \cap \mathfrak{g}_{F_{2, L}^{*}}^{\sigma,+}$. Now, let $e$ be a coset in $V_{F_{i, L}^{*}}^{\sigma}$. By the same result, there exists a lift $X \in \mathfrak{g}_{F_{1, L}^{*}} \cap \mathfrak{g}_{F_{2, L}^{*}}$. By Proposition III.9, we may project $X$ to $X_{\sigma} \in \mathfrak{g}_{F_{1, L}^{*}}^{\sigma}$. This is the desired representative which lies in $\mathfrak{g}_{F_{1, L}^{*}}^{\sigma} \cap \mathfrak{g}_{F_{2, L}^{*}}^{\sigma}$.

Remark III.41. Due to the previous result, whenever $F_{1, L}^{*}$ and $F_{2, L}^{*}$ are strongly $r$ associated, we are able to identify $V_{F_{1, L}^{*}}^{L}$ with $V_{F_{2, L}^{*}}^{L}$ and $V_{F_{1, L}^{*}}^{\sigma}$ with $V_{F_{2, L}^{*}}^{\sigma}$. We let $i^{L}$ and $i^{\sigma}$ denote the respective bijective identifications. 
Definition III.42. If $F_{L}^{*} \in \mathcal{F}_{L}(r)$ and $x \in F_{L}^{*}$, then the image of $H_{x}$ in $\operatorname{Aut}_{\mathfrak{f}}\left(V_{F_{L}^{*}}^{\sigma}\right)$ is denoted by $N_{x}^{\sigma}\left(F_{L}^{*}\right)$.

Lemma III.43. Suppose $F_{i, L}^{*} \in \mathcal{F}_{L}(r)$ and $x_{i} \in F_{i, L}^{*}$ for $i=1,2$. If $F_{1, L}^{*}$ and $F_{2, L}^{*}$ are strongly $r$-associated, then $N_{x_{i}}^{\sigma}\left(F_{i, L}^{*}\right)$ is the image of $H_{x_{1}} \cap H_{x_{2}}$ in $\operatorname{Aut}_{\mathfrak{f}}\left(V_{F_{i, L}^{*}}^{\sigma}\right)$ for $i=1,2$. Moreover,

$$
N_{x_{1}}^{\sigma}\left(F_{1, L}^{*}\right)=N_{x_{2}}^{\sigma}\left(F_{2, L}^{*}\right)
$$

under the identification induced by $i^{\sigma}$.

Proof. Let $\mathcal{A}$ be an apartment in $\mathcal{B}(H)$ containing $x_{1}$ and $x_{2}$. Choose $\psi \in \Psi(\mathcal{A})$ such that the image of $U_{\psi}$ in $\operatorname{Aut}_{\mathfrak{f}}\left(V_{F_{1, L}^{*}}^{\sigma}\right)$ is nontrivial and $\psi\left(x_{1}\right)=0$. We will show that $\psi\left(x_{2}\right)=0$.

Suppose $\psi\left(x_{2}\right)>0$. Since the image of $U_{\psi}$ in $\operatorname{Aut}_{f}\left(V_{F_{1, L}^{*}}^{\sigma}\right)$ is nontrivial, using the identification from Lemma III.40, there exists some $h \in U_{\psi}$ and $X \in \mathfrak{g}_{x_{1}, r}^{\sigma} \cap \mathfrak{g}_{x_{2}, r}^{\sigma}$ such that

$$
{ }^{h} X \neq X \bmod \mathfrak{g}_{x_{1}, r^{+}}^{\sigma} \cap \mathfrak{g}_{x_{2}, r^{+}}^{\sigma}
$$

On the other hand, we have ${ }^{h} X-X \in \mathfrak{g}_{x_{2}, r^{+}}^{\sigma}$, so by Lemma III.40, we have

$$
{ }^{h} X-X \in \mathfrak{g}_{x_{1, r}}^{\sigma} \cap \mathfrak{g}_{x_{2}, r^{+}}^{\sigma}=\mathfrak{g}_{x_{1}, r^{+}}^{\sigma} \cap \mathfrak{g}_{x_{2}, r^{+}}^{\sigma} .
$$

This is a contradiction, so we must have $\psi\left(x_{2}\right) \leq 0$.

Suppose $\psi\left(x_{2}\right)<0$. Since $x_{1}$ and $x_{2}$ lie in an affine space, we regard $v=x_{2}-x_{1}$ as a vector. Consider the function

$$
f_{v}: \mathbb{R} \rightarrow \mathbb{R}
$$


defined by $\epsilon \mapsto \psi\left(x_{1}+\epsilon v\right)$, where $x_{1}+\epsilon v$ is interpreted as the point $z \in \mathcal{A}$ for which $z-x_{1}=\epsilon v$. For all $\epsilon \in \mathbb{R}$, we have $x_{1}+\epsilon v \in \mathcal{A}$, so $f_{v}$ is well-defined. Since $\psi\left(x_{2}\right)<0$, we have

$$
f_{v}(1)=\psi\left(x_{2}\right)<0
$$

so, since $\psi$ is continuous, we must have $f_{v}(\epsilon)<0$ whenever $\epsilon>0$, and similarly $f_{v}(\epsilon)>0$ whenever $\epsilon<0$. Recall that $A\left(\mathcal{A}, F_{1, L}^{*}\right)=A\left(\mathcal{A}, F_{2, L}^{*}\right)$ by definition of strong $r$-associativity. Since $x_{1}, x_{2} \in A\left(\mathcal{A}, F_{2, L}^{*}\right)=A\left(\mathcal{A}, F_{1, L}^{*}\right)$, we must have $x_{1}+\mathbb{R} v \subset$ $A\left(\mathcal{A}, F_{1, L}^{*}\right)$. Thus, since $F_{1, L}^{*} \cap \mathcal{A}$ is open in $A\left(\mathcal{A}, F_{1, L}^{*}\right)=A\left(\mathcal{A}, F_{2, L}^{*}\right)$, there is some $\epsilon<0$ for which $x_{1}+\epsilon v \in F_{1, L}^{*} \cap \mathcal{A}$. In particular, by Lemma III.6, we have $\mathfrak{g}_{x_{1}+\epsilon v, r}^{\sigma}=\mathfrak{g}_{x_{1}, r}^{\sigma}$ and $\mathfrak{g}_{x_{1}+\epsilon v, r^{+}}^{\sigma}=\mathfrak{g}_{x_{1}, r^{+}}^{\sigma}$. Moreover, $\psi\left(x_{1}+\epsilon v\right)=f_{v}(\epsilon)>0$, so we have $U_{\psi} \subset H_{x_{1}+\epsilon v, 0^{+}}$ Thus, $h \in U_{\psi}$ acts trivially on $\mathfrak{g}_{x_{1}+\epsilon v, r}^{\sigma} / \mathfrak{g}_{x_{1}+\epsilon v, r^{+}}^{\sigma}=V_{F_{1, L}^{*}}^{\sigma}$, a contradiction.

We have thus shown that $\psi\left(x_{2}\right)=0$. If $\mathcal{A}$ corresponds to a maximal $k$-split torus $\mathbf{S}$ of $\mathbf{H}$, recall that $\mathbf{S}$ lies inside a maximal $k$-torus $\mathbf{Z}$ as described in Section II.3. Since the image of $H_{x_{1}}$ is determined by a filtration subgroup of $\mathbf{Z}$ (which also lies in $H_{x_{2}}$ ) and the $U_{\psi}$ 's, the proof shows that if $h \in H_{x_{1}}$ has nontrivial image in $\operatorname{Aut}_{\mathfrak{f}}\left(V_{F_{1, L}^{*}}^{\sigma}\right)$, then there exists some $h^{\prime} \in H_{x_{1}} \cap H_{x_{2}}$ for which the images of $h$ and $h^{\prime}$ in $\operatorname{Aut}_{\mathfrak{f}}\left(V_{F_{i, L}^{*}}^{\sigma}\right)$ coincide.

Definition III.44. Let $F_{L}^{*} \in \mathcal{F}_{L}(r)$ and $x \in F_{L}^{*}$. Define $N^{\sigma}\left(F_{L}^{*}\right) \subset \operatorname{Aut}_{\mathfrak{f}}\left(V_{F_{L}^{*}}^{\sigma}\right)$ by

$$
N^{\sigma}\left(F_{L}^{*}\right)=N_{x}^{\sigma}\left(F_{L}^{*}\right)
$$

\subsection{An equivalence relation}

Throughout this section, we shall fix an isotypic component $\mathfrak{g}^{\sigma}$ corresponding to an irreducible subrepresentation $\left(\sigma, V^{\sigma}\right)$ of $L$ in $\mathfrak{g}$ and suppress the symbol $\sigma$ in the notation. 


\section{Definition III.45.}

$$
I_{r}:=\left\{\left(F_{L}^{*}, v\right) \mid F_{L}^{*} \in \mathcal{F}_{L}(r) \text { and } v \in V_{F_{L}^{*}}^{\sigma}\right\}
$$

Let $x \in \mathcal{B}(H)$. For $v \in V_{x, r}$, we interpret ${ }^{h} v$ as the image of ${ }^{h} X$, where $X$ is a lift of $v$ in $\mathfrak{g}_{x, r}$.

Definition III.46. For $\left(F_{1, L}^{*}, v_{1}\right)$ and $\left(F_{2, L}^{*}, v_{2}\right)$ in $I_{r}$, we write $\left(F_{1, L}^{*}, v_{1}\right) \sim\left(F_{2, L}^{*}, v_{2}\right)$ provided that there exists some $h \in H$ and an apartment $\mathcal{A} \subset \mathcal{B}(H)$, for which $F_{1, L}^{*} \cap \mathcal{A}, F_{2, L}^{*} \cap \mathcal{A}$ are nonempty, and

1. $A\left(\mathcal{A}, F_{1, L}^{*}\right)=A\left(\mathcal{A}, h F_{2, L}^{*}\right)$

2. $v_{1}={ }^{h} v_{2}$ in $V_{F_{1, L}^{*}}^{\sigma}=V_{h F_{2, L}^{*}}^{\sigma}$,

where we use the usual identification from Lemma III.40 for the second condition.

Lemma III.47. The relation defined above is an equivalence relation on $I_{r}$.

Proof. For reflexivity, let $h=1$.

Now, suppose $\left(F_{1, L}^{*}, v_{1}\right) \sim\left(F_{2, L}^{*}, v_{2}\right)$. By definition, there exists an apartment $\mathcal{A} \subset \mathcal{B}(H)$ and an element $h \in H$ such that

1. $A\left(\mathcal{A}, F_{1, L}^{*}\right)=A\left(\mathcal{A}, h F_{2, L}^{*}\right) \neq \emptyset$ and

2. $v_{1}={ }^{h} v_{2}$ in $V_{F_{1, L}^{*}}^{\sigma}=V_{h F_{2, L}^{*}}^{\sigma}$.

Since $h^{-1} A\left(\mathcal{A}, F_{1, L}^{*}\right)=A\left(h^{-1} \mathcal{A}, h^{-1} F_{2, L}^{*}\right)$, we have

1. $A\left(h^{-1} \mathcal{A}, h^{-1} F_{1, L}^{*}\right)=A\left(h^{-1} \mathcal{A}, F_{2, L}^{*}\right) \neq \emptyset$ and

2. ${ }^{h^{-1}} v_{1}=v_{2}$ in $V_{h^{-1} F_{1, L}^{*}}^{\sigma}=V_{F_{2, L}^{*}}^{\sigma}$. 
In particular, $\left(F_{2, L}^{*}, v_{2}\right) \sim\left(F_{1, L}^{*}, v_{1}\right)$.

For transitivity, suppose $\left(F_{1, L}, v_{1}\right),\left(F_{2, L}^{*}, v_{2}\right),\left(F_{3, L}^{*}, v_{3}\right) \in I_{r}$ such that $\left(F_{1, L}^{*}, v_{1}\right) \sim$ $\left(F_{2, L}^{*}, v_{2}\right)$ and $\left(F_{2, L}^{*}, v_{2}\right) \sim\left(F_{3, L}^{*}, v_{3}\right)$. By definition, there exist $h_{2}, h_{3} \in H$ and apartments $\mathcal{A}_{12}, \mathcal{A}_{23} \subset \mathcal{B}(H)$ such that

$$
\begin{aligned}
& A\left(\mathcal{A}_{12}, F_{1, L}^{*}\right)=A\left(\mathcal{A}_{12}, h_{2} F_{2, L}^{*}\right) \neq \emptyset \\
& A\left(\mathcal{A}_{23}, F_{2, L}^{*}\right)=A\left(\mathcal{A}_{23}, h_{3} F_{3, L}^{*}\right) \neq \emptyset
\end{aligned}
$$

and

$$
\begin{aligned}
& v_{1}={ }^{h_{2}} v_{2} \text { in } V_{F_{1, L}^{*}}^{\sigma}=V_{h_{2} F_{2, L}^{*}}^{\sigma} \\
& v_{2}={ }^{h_{3}} v_{3} \text { in } V_{F_{2, L}^{*}}^{\sigma}=V_{h_{3} F_{3, L}^{*}}^{\sigma} .
\end{aligned}
$$

Fix $x_{i} \in C\left(F_{i, L}^{*}\right)$. The first and second lines show that

$$
A\left(\mathcal{A}_{12}, h_{2} F_{2, L}^{*}\right), A\left(h_{2} \mathcal{A}_{23}, h_{2} F_{2, L}^{*}\right) \neq \emptyset .
$$

In particular, $\mathcal{A}_{12} \cap h_{2} F_{2, L}^{*}, h_{2} \mathcal{A}_{23} \cap h_{2} F_{2, L}^{*} \neq \emptyset$, so there exists an element $h \in H_{h_{2} x_{2}} \subset$ $\operatorname{stab}_{H}\left(h_{2} F_{2, L}^{*}\right)$ such that $h \mathcal{A}_{12}=h_{2} \mathcal{A}_{23}$. We have

$$
\begin{aligned}
\emptyset \neq A\left(\mathcal{A}_{12}, F_{1, L}^{*}\right) & =A\left(\mathcal{A}_{12}, h_{2} F_{2, L}^{*}\right)=h^{-1} A\left(h \mathcal{A}_{12}, h h_{2} F_{2, L}^{*}\right) \\
& =h^{-1} A\left(h_{2} \mathcal{A}_{23}, h_{2} F_{2, L}^{*}\right)=h^{-1} h_{2} A\left(\mathcal{A}_{23}, h_{3} F_{3, L}^{*}\right) \\
& =A\left(\mathcal{A}_{12}, h^{-1} h_{2} h_{3} F_{3, L}^{*}\right) .
\end{aligned}
$$


Now, by the proof of [[12], 3.5.1] and Lemma III.40, we have a surjection

$$
\mathfrak{g}_{F_{1, L}^{*}}^{\sigma} \cap \mathfrak{g}_{h_{2} F_{2, L}^{*}}^{\sigma} \cap \mathfrak{g}_{h^{-1} h_{2} h_{3} F_{3, L}^{*}}^{\sigma} \rightarrow V_{F_{1, L}^{*}}^{\sigma}
$$

As a result, there is some $X \in \mathfrak{g}_{F_{1, L}^{*}}^{\sigma} \cap \mathfrak{g}_{h_{2} F_{2, L}^{*}}^{\sigma} \cap \mathfrak{g}_{h^{-1} h_{2} h_{3} F_{3, L}^{*}}^{\sigma}$ such that the image of $X$ in $V_{F_{1, L}^{*}}^{\sigma}$ is $v_{1}$. Since ${ }^{h_{2}} v_{2}=v_{1}$ under the standard identification, we have that

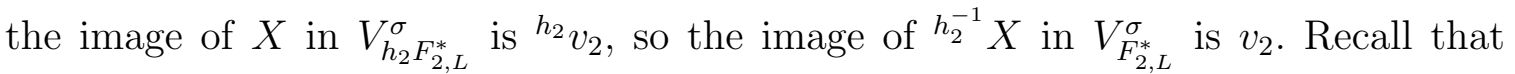
$h \in H_{h_{2} x_{2}}=\operatorname{Int}\left(h_{2}\right) H_{x_{2}}$, so $h_{2}^{-1} h h_{2} \in H_{x_{2}}$. Thus, the image of ${ }^{-1} h X={ }^{\left(h_{2}^{-1} h h_{2}\right) h_{2}^{-1} X}$ in $V_{F_{2, L}^{*}}^{\sigma}$ is ${ }^{h_{2}^{-1} h h_{2}} v_{2}$. By the previous computation and the fact that $X$ was chosen in $\mathfrak{g}_{h^{-1} h_{2} h_{3} F_{3, L}^{*}}^{\sigma}$, we have ${ }^{h_{2}^{-1} h} X \in \mathfrak{g}_{F_{2, L}^{*}}^{\sigma} \cap \mathfrak{g}_{h_{3} F_{3, L}^{*}}^{\sigma}$. Since $F_{2, L}^{*}$ and $h_{3} F_{3, L}^{*}$ are strongly $r$ associated, by Lemma III.43, $N^{\sigma}\left(F_{2, L}^{*}\right)=N^{\sigma}\left(h_{3} F_{3, L}^{*}\right)$. Thus, again by Lemma III.43, there is an element $h^{\prime} \in H_{h_{3} x_{3}} \cap H_{x_{2}}$ such that

$$
h_{2}^{-1} h h_{2} v_{2}={ }^{h^{\prime}} v_{2}={ }^{h} h_{3} v_{3} \text { in } V_{F_{2, L}^{*}}^{\sigma}=V_{h_{3} F_{3, L}^{*}}
$$

As a consequence, the image of $X$ in $V_{h^{-1} h_{2} h_{3} F_{3, L}^{*}}^{\sigma}$ is

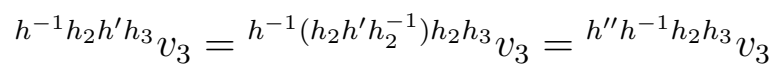

with $h^{\prime \prime} \in \operatorname{Int}\left(h^{-1} h_{2}\right)\left(H_{h_{3} x_{3}} \cap H_{x_{2}}\right) \subset H_{h^{-1} h_{2} h_{3} x_{3}}$. Since

$$
h^{\prime \prime} \in H_{h^{-1} h_{2} h_{3} x_{3}} \subset \operatorname{stab}_{H}\left(h^{-1} h_{2} h_{3} F_{3, L}^{*}\right),
$$

we have

$$
A\left(\mathcal{A}_{12}, F_{1, L}^{*}\right)=A\left(\mathcal{A}_{12}, h^{-1} h_{2} h_{3} F_{3, L}^{*}\right)=A\left(\mathcal{A}_{12}, h^{\prime \prime} h^{-1} h_{2} h_{3} F_{3, L}^{*}\right) \neq \emptyset .
$$

Moreover, 


$$
v_{1}={ }^{h_{2}} v_{2}={ }^{h^{\prime \prime} h^{-1} h_{2} h_{3}} v_{3} \text { in } V_{F_{1, L}^{*}}^{\sigma}=V_{h^{\prime \prime} h^{-1} h_{2} h_{3} F_{3, L}^{*}}^{\sigma}
$$

This shows that $\left(F_{1, L}^{*}, v_{1}\right) \sim\left(F_{3, L}^{*}, v_{3}\right)$. 


\section{CHAPTER IV}

\section{Jacobson-Morosov triples}

Fix $r \in \mathbb{R}$. Before attaching a nilpotent $H$-orbit to the types of pairs discussed at the end of Chapter III, we will need a way to pass from $\mathfrak{s l}_{2}(\mathfrak{f})$-triples to $\mathfrak{s l}_{2}(k)$-triples and vice versa. In this section, we describe this procedure in detail. Recall that $\mathcal{N}$ denotes the set of nilpotent elements in $\mathfrak{g}$ as defined in the preliminaries. For the first part of this chapter, we shall fix an isotypic component $\mathfrak{g}^{\sigma}$ associated to an irreducible subrepresentation $\left(\sigma, V^{\sigma}\right)$ of $L$ in $\mathfrak{g}$. As in Lemma III.26, we will identify $\mathfrak{g}_{x, r}^{\sigma} / \mathfrak{g}_{x, r^{+}}^{\sigma}$ with $V_{x, r}^{\sigma}$.

Definition IV.1. Let $F_{L}^{*} \in \mathcal{F}_{L}(r)$. An element $e \in V_{F_{L}^{*}}^{\sigma}$ is called degenerate provided that there exists a lift $E \in \mathfrak{g}_{F_{L}^{*}}^{\sigma} \cap \mathcal{N}$.

The following lemma gives us an alternate characterization of degenerate elements.

Lemma IV.2. Fix $F_{L}^{*} \in \mathcal{F}_{L}(r)$. An element $e \in V_{F_{L}^{*}}^{\sigma}$ is degenerate if and only if zero lies in the Zariski closure of ${ }^{H_{x}}$ e for all $x \in F_{L}^{*}$.

Proof. " $\Rightarrow "$ : We refer to [[21], Proposition 4.3]. Fix $x \in F_{L}^{*}$ and a lift $E \in \mathfrak{g}_{x, r}^{\sigma} \cap \mathcal{N}$. In the notation of [[21], Proposition 4.3], we take $V=\mathfrak{g}_{x, r}^{\sigma}, W=\mathfrak{g}_{x, r^{+}}^{\sigma}$, and let $\rho: H_{x} \rightarrow G L(V)$ be the rational representation given by the adjoint action of $H_{x}$ on $\mathfrak{g}^{\sigma}$ restricted to the lattice $\mathfrak{g}_{x, r}^{\sigma}$. We note that $\varpi \mathfrak{g}_{x, r}^{\sigma}=\mathfrak{g}_{x, r+1}^{\sigma} \subset \mathfrak{g}_{x, r^{+}}^{\sigma}$, and $E$ is a nilpotent lift of $e$, so all hypotheses are satisfied. From [[21], Proposition 4.3], 
we conclude that zero lies in the Zariski closure of ${ }^{\mathrm{H}_{x}} e$, with respect to the induced representation of $\rho$ from $\mathrm{H}_{x}$ to $G L(V / W)$.

" $\Leftarrow "$ : Fix $x \in F_{L}^{*}$. Let $\mathbf{S}$ be a maximal $k$-split torus in $\mathbf{H}$ with $x \in \mathcal{A}(\mathbf{S}, k)$. We consider $V_{F_{L}^{*}}^{\sigma}$ as the vector space of $\mathrm{f}$-rational points of the affine $\mathrm{H}_{x}$-scheme $\operatorname{Lie}\left(\mathrm{G}_{x}\right)^{\sigma}$. Then, by [[16], Theorem 1.4], there exists a one-parameter subgroup $\bar{\lambda} \in \mathbf{X}_{*}^{\mathfrak{f}}\left(\mathbf{H}_{x}\right)$ such that

$$
\lim _{t \rightarrow 0} \bar{\lambda}(t) e=0
$$

Let $\mathbf{S}$ be the maximal $\mathfrak{f}$-split torus in $\mathrm{H}_{x}$ corresponding to $\mathrm{S}$. Then, since $\mathrm{H}_{x}(\mathfrak{f})$ acts transitively on the set of maximal $\mathfrak{f}$-split tori in $\mathrm{H}_{x}$, there exists an element $\bar{h} \in \mathrm{H}_{x}(\mathfrak{f})$ and a one-parameter subgroup $\bar{\mu} \in \mathbf{X}_{*}^{\mathfrak{f}}(\mathrm{S})$ such that

$$
\lim _{t \rightarrow 0} \bar{\mu}(t) \bar{h} e=0 .
$$

Let $\mu \in \mathbf{X}_{*}(\mathbf{S})$ be a lift of $\bar{\mu}$ and let $h \in H_{x}$ be a lift of $\bar{h}$. Also, let $E^{\prime}$ be a lift of $e$ in $\mathfrak{g}_{x, r}^{\sigma}$. Without loss of generality, we may assume that

$$
{ }^{h} E^{\prime}=\sum_{\psi} X_{\psi}
$$

where $X_{\psi} \in \mathfrak{g}_{\psi} \cap \mathfrak{g}^{\sigma}$ and $\psi(x)=r$. We claim that $\psi(x+\epsilon \cdot \mu)>r$ for all $\psi$ appearing in the sum. This will happen precisely when $\langle\mu, \dot{\psi}\rangle>0$ since

$$
\psi(x+\epsilon \mu)=\psi(x)+\epsilon \cdot\langle\mu, \dot{\psi}\rangle=r+\epsilon \cdot\langle\mu, \dot{\psi}\rangle .
$$

Note, however, that

$$
0=\lim _{t \rightarrow 0} \bar{\mu}(t) \bar{h} \overline{E^{\prime}}=\sum \lim _{t \rightarrow 0} \bar{\mu}(t) \overline{X_{\psi}}=\sum \lim _{t \rightarrow 0} t^{\langle\mu, \dot{\psi}\rangle} \overline{X_{\psi}}
$$

so, in particular, the limit is 0 if and only if $\langle\mu, \dot{\psi}\rangle>0$. This shows that ${ }^{h} E^{\prime} \in \mathfrak{g}_{x+\epsilon \mu, r^{+}}^{\sigma}$. 
For $\epsilon$ sufficiently small, $x+\epsilon \mu$ lies in a generalized $(r, L)$-facet $C_{L}^{*}$ containing $F_{L}^{*}$ in its closure. By [[12], Corollary 3.2.19], we have $\mathfrak{g}_{x, r^{+}}^{\sigma} \subset \mathfrak{g}_{x+\epsilon \mu, r^{+}}^{\sigma}$. Thus,

$$
{ }^{h}\left(E^{\prime}+\mathfrak{g}_{x, r^{+}}^{\sigma}\right)={ }^{h}\left(E^{\prime}+\mathfrak{g}_{F_{L}^{*}}^{\sigma,+}\right) \subset \mathfrak{g}_{x+\epsilon \cdot \mu, r^{+}}^{\sigma}
$$

for $\epsilon$ taken to be sufficiently small. We have shown that the coset $E^{\prime}+\mathfrak{g}_{x, r}^{\sigma,+}$ lies in $\mathfrak{g}_{r^{+}}$as defined in [[2], 3.2.5]. In particular, by [[2], Corollary 3.2.6], $e$ is a degenerate coset.

In order to discuss $\mathfrak{s l}_{2}(\mathfrak{f})$-triples, we next introduce an $\mathfrak{f}$-Lie algebra $\overline{\mathfrak{g}}_{x}$ which is associated to a point $x \in \mathcal{B}(H)$. In the preliminaries, we chose a uniformizer $\varpi$ for $k$, which allows us to identify $V_{x, s}$ with $V_{x, s+j \cdot \ell}$ where $L$ is the splitting field of $\mathbf{G}$ containing $K$, and $\ell=[L: K]$. Using this identification, we define

$$
\overline{\mathfrak{g}}_{x}:=\bigoplus_{s \in \mathbb{R} / \ell \cdot \mathbb{Z}} V_{x, s} .
$$

If $\bar{X}_{s} \in V_{x, s}$ and $\bar{X}_{t} \in V_{x, t}$, then define $\left[\overline{X_{s}}, \overline{X_{t}}\right]$ to be the image of $\left[X_{s}, X_{t}\right] \in \mathfrak{g}_{x,(s+t)}$ in $V_{x,(s+t)}$ where $X_{s} \in \mathfrak{g}_{x, s}$ and $X_{t} \in \mathfrak{g}_{x, t}$ are any lifts of $\overline{X_{s}}$ and $\overline{X_{t}}$ respectively. We can then linearly extend to obtain a well-defined bracket on all of $\overline{\mathfrak{g}}_{x}$. With this product, $\overline{\mathfrak{g}}_{x}$ is an $\mathfrak{f}$-Lie algebra.

\subsection{Some hypotheses}

From this point on, we assume that $L$ is a finite cyclic group of order $m$. We restrict our attention to nilpotent elements in $\mathfrak{g}^{1}$, the 1-eigenspace in $\mathfrak{g}$. The invariant theory of $H$ acting on $\mathfrak{g}^{1}$ was initiated by Vinberg, and for applications to representation theory, this is the case with which the main result is proven. Namely, we parametrize nilpotent $H$-orbits in $\mathfrak{g}^{1}$. We now list some hypotheses (which occur also in [[12]]) 
needed in order to utilize the theory of $\mathfrak{s l}_{2}$-triples. These hypotheses hold under mild restrictions on $\mathbf{G}$ and $k$, and we give some references for more details on when each hypothesis is valid. We note that when the characteristic of $\mathfrak{f}$ is zero, all hypotheses hold.

Before stating the first hypothesis, we set up a framework for $\mathfrak{s l}_{2}$-triples with respect to the $L$-action. In particular, suppose $X \in \mathcal{N} \cap \mathfrak{g}^{1}$. Since $\left[\mathfrak{g}^{1}, \mathfrak{g}^{-1}\right] \subset \mathfrak{h}$, we will primarily be interested in $\mathfrak{s l}_{2}(k)$-triples $\{Y, H, X\}$ for which $X \in \mathfrak{g}^{1}, Y \in \mathfrak{g}^{-1}$, and $H \in \mathfrak{h}$. We will define such triples below. Let $V_{x,-r}^{i}$ denote the quotient $\mathfrak{g}_{F_{L}^{*}}^{i} / \mathfrak{g}_{F_{L}^{*}}^{i,+}$, for $i=-1,0,1$. Also, let $\left(\overline{\mathfrak{g}}_{x}\right)^{i}$ denote the $(-1), 0$, or 1-eigenspaces in $\overline{\mathfrak{g}}_{x}$.

Hypothesis IV.3. Suppose $x \in \mathcal{B}(H)$. If $X \in \mathcal{N} \cap\left(\mathfrak{g}_{x, r}^{1} \mid \mathfrak{g}_{x, r^{+}}^{1}\right)$, then there exist $H \in \mathfrak{h}_{x, 0}$ and $Y \in \mathfrak{g}_{x,-r}^{-1}$ such that

$$
\begin{aligned}
{[H, X] } & =2 X \bmod \mathfrak{g}_{x, r^{+}}^{1} \\
{[H, Y] } & =-2 Y \bmod \mathfrak{g}_{x,(-r)^{+}}^{-1} \\
{[X, Y] } & =H \bmod \mathfrak{h}_{x, 0^{+}} .
\end{aligned}
$$

If $(f, h, e)$ denotes the image of $(Y, H, X)$ in $V_{x,-r}^{-1} \times V_{x, 0}^{0} \times V_{x, r}^{1} \subset \overline{\mathfrak{g}}_{x}$, then $\{f, h, e\}$ is an $\mathfrak{s l}_{2}(\mathfrak{f})$-triple, and $\overline{\mathfrak{g}}_{x}$ decomposes into a direct sum of irreducible $\langle f, h, e\rangle$-modules of highest weight at most $p-3$. Moreover, there exists some $\bar{\lambda} \in \mathbf{X}_{*}^{\mathfrak{f}}\left(\mathrm{H}_{x}\right)$, uniquely determined up to an element of $\mathbf{X}_{*}\left(\mathbf{Z}_{x}\right)$ whose differential is zero, such that the following hold:

1. The image of $d \bar{\lambda}$ in $\operatorname{Lie}\left(\mathrm{H}_{x}\right)$ coincides with the subspace spanned by $h$.

2. Suppose $i \in \mathbb{Z}$. For $v \in \overline{\mathfrak{g}}_{x}$

$$
\text { if }^{\bar{\lambda}(t)} v=t^{i} v \text {, then }|i| \leq p-3 \text { and } \operatorname{ad}(h) v=i v .
$$


More details on Hypothesis IV.3 can be found in [[12], Appendix A].

Following [[19], I.2], and [[23], Section 3], we define a normal $\mathfrak{s l}_{2}$-triple below.

Definition IV.4. Let $\{Y, H, X\}$ (resp. $\{f, h, e\})$ be an $\mathfrak{s l}_{2}(k)$-triple in $\mathfrak{g}\left(\operatorname{resp} . \mathfrak{s l}_{2}(\mathfrak{f})\right.$ triple in $\left.\overline{\mathfrak{g}}_{x}\right)$. We call $\{Y, H, X\}$ (resp. $\left.\{f, h, e\}\right)$ a normal $\mathfrak{s l}_{2}(k)$-triple (resp. $\mathfrak{s l}_{2}(\mathfrak{f})$ triple) provided that $X \in \mathfrak{g}^{1}, Y \in \mathfrak{g}^{-1}$ (resp. $e \in\left(\overline{\mathfrak{g}}_{x}\right)^{1}, f \in\left(\overline{\mathfrak{g}}_{x}\right)^{-1}$ ) and $H \in \mathfrak{h}$ (resp. $\left.h \in\left(\overline{\mathfrak{g}}_{x}\right)^{0}\right)$.

Remark IV.5. We note that if $\{f, h, e\}$ is any $\mathfrak{s l}_{2}(\mathfrak{f})$-triple in $\overline{\mathfrak{g}}_{x}$ with $e \in V_{x, r}^{1}$, then we may always choose a normal triple completing $e$. By projecting $h$ to $V_{x, 0}$, we may assume $h \in V_{x, 0}$. By Lemma III.26, we may write $h=h_{0}+\sum_{i} h_{i}$ where $h_{0} \in V_{x, 0}^{0}$ and $h_{i} \in V_{x, 0}^{i}, i=1, \ldots m-1$. We have $[h, e]=2 e \in V_{x, r}^{1}$, so $[h, e]=\left[h_{0}, e\right]+\sum_{i}\left[h_{i}, e\right]=$ 2e. By Lemma III.26, we have $\sum_{i}\left[h_{i}, e\right]=0$ since $\left[V_{x, 0}^{0}, V_{x, r}^{i}\right] \subset V_{x, r}^{i}$. By a similar argument, we have $f \in V_{x,-r}^{-1}$, so we may assume $\{f, h, e\}$ is a normal triple. This also shows that $h$ is $L$-fixed. In particular, the one-parameter subgroup $\bar{\lambda} \in \mathbf{X}_{*}^{\mathfrak{f}}\left(\mathrm{G}_{x}\right)$ has image inside $\left(G_{x}^{L}\right)^{\circ}$, and hence $H_{x}$ by Proposition A.6.

Definition IV.6. Keeping the above notation, we say that $\bar{\lambda} \in \mathbf{X}_{*}^{\mathfrak{f}}\left(\mathbf{H}_{x}\right)$ is adapted to the $\mathfrak{s l}_{2}(\mathfrak{f})$-triple obtained from the image of $(Y, H, X)$ in $V_{x,-r} \times V_{x, 0} \times V_{x, r}$.

Hypothesis IV.7. If $X \in \mathcal{N}^{1}$, then there exists some $m \in \mathbb{N}$ with $m \leq p-2$ such that $\operatorname{ad}(X)^{m}=0$.

Hypothesis IV.8. Choose $m \in \mathbb{N}$ (as in Hypothesis IV.7) so that $\operatorname{ad}(X)^{m}=0$ for all $X \in \mathcal{N}^{1}$. Suppose the characteristic of $k$ is zero or greater than $m$. Then there exists a $G$-equivariant map exp: $\mathcal{N} \rightarrow \mathcal{U}$ such that for all $X \in \mathcal{N}$, the adjoint action of $\exp (X)$ on $\mathfrak{g}$ is given by:

$$
\operatorname{Ad}(\exp (X))=\sum_{i=0}^{m} \frac{(\operatorname{ad}(X))^{i}}{i !} .
$$


In the next hypothesis, we use the letter $H$ in two different contexts. In the first occurrence, it appears as an element of $\mathfrak{g}$ which is part of an $\mathfrak{s l}_{2}(k)$-triple. In the last line of the hypothesis, it occurs as the group of $k$-rational points of $\mathbf{H}$. This notation is unfortunate, but in most cases, the meaning of this symbol will be clear from context.

Hypothesis IV.9. Suppose Hypothesis IV.8 holds. Let $X \in \mathcal{N}^{1}$. There exists a normal $\mathfrak{s l}_{2}(k)$-triple completing $X$. Moreover, if $\{Y, H, X\}$ is a normal $\mathfrak{s l}_{2}(k)$-triple completing $X$, then there is an algebraic group homomorphism $\varphi: \mathbf{S L}_{2} \rightarrow \mathbf{G}$ defined over $k$ such that $d \varphi\left(\begin{array}{ll}0 & 1 \\ 0 & 0\end{array}\right)=X, d \varphi\left(\begin{array}{ll}0 & 0 \\ 1 & 0\end{array}\right)=Y, d \varphi\left(\begin{array}{cc}1 & 0 \\ 0 & -1\end{array}\right)=H$, and for all $t \in k$,
1. $\varphi\left(\begin{array}{ll}1 & t \\ 0 & 1\end{array}\right)=\exp (t X)$ and
2. $\varphi\left(\begin{array}{ll}1 & 0 \\ t & 1\end{array}\right)=\exp (t Y)$

Lastly, (see below), any two normal $\mathfrak{s l}_{2}(k)$-triples which complete $X$ are conjugate by an element of $C_{H}(X)$.

Proposition IV.10. Assume Hypotheses IV.3 and IV.8 hold. If $\left\{Y^{\prime}, H^{\prime}, X\right\}$ and $\{Y, H, X\}$ are two normal $\mathfrak{s l}_{2}(k)$-triples completing $X$, then there exists an element $h \in C_{H}(X)$ for which $Y^{\prime}={ }^{h} Y$ and $H^{\prime}={ }^{h} H$.

Proof. (a generalization of an argument of Kostant) In order to verify the claim, we slightly modify the notation and argument given in [[18], Theorem 3.6] and [[7], Lemma 3.4.7].

Define $\mathfrak{h}_{X}:=\left[\mathfrak{g}^{-1}, X\right] \cap C_{\mathfrak{h}}(X)$, and let $U, V \in \mathfrak{h}_{X}$. Since $V=[W, X]$, for some $W \in \mathfrak{g}^{-1}$, and since $U$ centralizes $X$, we have

$$
[U, V]=[U,[W, X]]=[X,[W, U]]
$$


Since $W \in \mathfrak{g}^{-1}$ and $U \in \mathfrak{h}$, it follows that $[W, U] \in \mathfrak{g}^{-1}$, so $[U, V] \in\left[\mathfrak{g}^{-1}, X\right]$. This shows $\mathfrak{h}_{X}$ is a Lie subalgebra of $\mathfrak{g}$.

Kostant also shows that every element of $\mathfrak{g}_{X}:=[\mathfrak{g}, X] \cap C_{\mathfrak{g}}(X)$ is nilpotent. It follows that every element of $\mathfrak{h}_{X}$ is nilpotent. (Note that $\mathfrak{h}_{X}$ is also invariant under $\operatorname{ad}(H)$.) By Hypothesis IV.8, the adjoint action of $\exp (W)$ for $W \in \mathfrak{h}_{X}$ on an element of $\mathfrak{g}$ is given by $\operatorname{Ad}(\exp (W))=\sum_{i} \frac{(\operatorname{ad}(W))^{i}}{i !}$. In particular, we have

$$
\operatorname{Ad}(\exp (W))(H)=\sum_{i} \frac{(\operatorname{ad}(W))^{i}(H)}{i !} \in H+\mathfrak{h}_{X}
$$

We will show that for every $V \in \mathfrak{h}_{X}$, there exists some $W \in \mathfrak{h}_{X}$ such that $\operatorname{Ad}(\exp (W))(H)=H+V$. Define $C_{\mathfrak{h}}(X)(i):=\left\{Z \in C_{\mathfrak{h}}(X) \mid[H, Z]=i Z\right\}$. Kostant shows that $\mathfrak{g}_{X} \subset \oplus_{i=1}^{m} C_{\mathfrak{g}}(X)(i)$ for some natural number $m$. In particular, $\mathfrak{h}_{X} \subset$ $\oplus_{i=1}^{m} C_{\mathfrak{h}}(X)(i)$. We now construct the element $W$ inductively.

Set $W_{1}=-V_{1}$, where $V_{1}$ is the component of $V$ lying in $C_{\mathfrak{h}}(X)(1)$. Then, $W_{1}$ lies in $\mathfrak{h}_{X}$, and we have $\operatorname{ad}\left(W_{1}\right)(H)=-\left[H, W_{1}\right]=-W_{1}=V_{1}$. Again, using Hypothesis IV.8, we have

$$
\begin{aligned}
\operatorname{Ad}\left(\exp \left(W_{1}\right)\right)(H)-(H+V) & =\sum_{i=0}^{m} \frac{\left(\operatorname{ad}\left(W_{1}\right)\right)^{i}(H)}{i !}-(H+V) \\
& =\left(V_{1}-V\right)+\sum_{i=2}^{m} \frac{\left(\operatorname{ad}\left(W_{1}\right)\right)^{i}(H)}{i !} \in \bigoplus_{i \geq 2} C_{\mathfrak{h}}(X)(i)
\end{aligned}
$$

The last line results from the fact that the restriction of $\operatorname{ad}(H)$ to $\mathfrak{h}_{X}$ takes strictly positive integral values as eigenvalues.

Thus, we have verified the base case. We now assume that we have constructed elements $W_{j}$ such that

1. $W_{j} \in \bigoplus_{1 \leq i \leq j} C_{\mathfrak{h}}(X)(i)$

2. $\operatorname{Ad}\left(\exp \left(W_{j}\right)\right)(H)-(H+V) \in \bigoplus_{j+1 \leq i \leq m} C_{\mathfrak{h}}(X)(i)$. 
Now, let $W_{j+1}^{\prime}$ be the component of $\operatorname{Ad}\left(\exp \left(W_{j}\right)\right)(H)-(H+V)$ which lies in $C_{\mathfrak{h}}(X)(j+$ 1).

Letting $W_{j+1}=W_{j}+\frac{1}{j+1} W_{j+1}^{\prime}$, it is clear that $W_{j+1} \in \oplus_{1 \leq i \leq j+1} C_{\mathfrak{h}}(X)(i)$. Moreover, we have

$$
\begin{aligned}
\operatorname{Ad}\left(\exp \left(W_{j+1}\right)\right)(H)-(H+V) & =\sum_{i=0}^{m} \frac{\left(\operatorname{ad}\left(W_{j+1}\right)\right)^{i}(H)}{i !}-(H+V) \\
& =H+\left[W_{j+1}, H\right]+\cdots-(H+V) \\
& =H+\left[W_{j}, H\right]+\frac{1}{j+1}\left[W_{j+1}^{\prime}, H\right]+\cdots-(H+V) \\
& =H+\left[W_{j}, H\right]-W_{j+1}^{\prime}+\cdots-(H+V)
\end{aligned}
$$

Only the terms with indices up to $i=1$ have been expanded in the last line written. If we expand higher terms, we obtain a sum of the form

$H+\left[W_{j}, H\right]-W_{j+1}^{\prime}+\frac{\left[W_{j},\left[W_{j}, H\right]\right]}{2 !}+\frac{\left[\frac{1}{j+1} W_{j+1}^{\prime},\left[W_{j}, H\right]\right]}{2 !}-\frac{\left[W_{j+1}, W_{j+1}^{\prime}\right]}{2 !} \cdots-(H+V)$

so after expansion of higher order terms, we may simplify the previous line to the sum of $\operatorname{Ad}\left(\exp \left(W_{j}\right)\right)(H)-(H+V),-W_{j+1}^{\prime}$, and terms which lie in weight spaces of $C_{\mathfrak{h}}(X)$ with weights greater than or equal to $(j+2)$. Thus, by definition of $W_{j+1}^{\prime}$, we have

$$
\operatorname{Ad}\left(\exp \left(W_{j+1}\right)\right)(H)-(H+V) \in \bigoplus_{j+2 \leq i \leq m} C_{\mathfrak{h}}(X)(i) .
$$

Finally, letting $W=W_{m}$, we have $\operatorname{Ad}(\exp (W))(H)=H+V$.

Now, since $\left[H^{\prime}, X\right]=2 X=[H, X]$, we have $H^{\prime}-H \in C_{\mathfrak{h}}(X)$. On the other hand, since $\left[X, Y^{\prime}-Y\right]=H^{\prime}-H$, we have $H^{\prime}-H \in\left[\mathfrak{g}^{-1}, X\right]$. In particular, we have $H^{\prime}-H \in \mathfrak{h}_{X}$. By the argument above, there is some $W \in \mathfrak{h}_{X}$ such that

$$
\operatorname{Ad}(\exp (W))(H)=H+\left(H^{\prime}-H\right)=H^{\prime} .
$$


By the construction of the element $W$ in the proof, it is clear that $W$ lies in $\mathfrak{h}$, so since exp takes $\mathfrak{h} \cap \mathcal{N}$ into $H$, we set $h=\exp (W)$.

Hypothesis IV.11. Let $x \in \mathcal{B}(H)$. For all $s \in \mathbb{R}_{>0}$ and for all $t \in \mathbb{R}$, there exists a map $\phi_{x}: \mathfrak{h}_{x, s} \rightarrow H_{x, s}$ such that for $V \in \mathfrak{h}_{x, s}$ and $W \in \mathfrak{g}_{x, t}^{1}$ we have

$$
{ }^{\phi_{x}(V)} W=W+[V, W] \bmod \mathfrak{g}_{x,(s+t)^{+}}^{1}
$$

Hypothesis IV.11 appears in [[1], Proposition 1.6.3] which is useful in [[12]]. The types of maps which are suitable for [[12]] might not generally work in the setting of finite (cyclic) group actions which we treat. However, the exponential map described in [[11], Appendix B] is sufficient for our purposes and satisfies this hypothesis.

\subsubsection{Obtaining $\mathfrak{s l}_{2}(k)$-triples from $\mathfrak{s l}_{2}(\mathfrak{f})$-triples}

Our next step will be to show how to obtain a normal $\mathfrak{s l}_{2}(k)$-triple from a normal $\mathfrak{s l}_{2}(\mathfrak{f})$-triple. We first recall the setup.

Let $x \in \mathcal{B}(H)$ and suppose $(f, h, e) \subset V_{x,-r}^{-1} \times V_{x, 0}^{0} \times V_{x, r}^{1} \subset \overline{\mathfrak{g}}_{x}$ is a nontrivial normal $\mathfrak{s l}_{2}(\mathfrak{f})$-triple. Suppose $\bar{\mu} \in \mathbf{X}_{*}^{\mathfrak{f}}\left(\mathbf{H}_{x}\right)$ is adapted to $\{f, h, e\}$. Let $\mathbf{S}$ be a maximal $k$-split torus of $\mathbf{H}$ such that $x \in \mathcal{A}(\mathbf{S}, k)$. Let $\mathbf{S}$ be the maximal $\mathfrak{f}$-split torus in $\mathbf{H}_{x}$ corresponding to $\mathbf{S}$. Since $\mathbf{H}_{x}$ is a reductive group over $\mathfrak{f}$, all maximal $\mathfrak{f}$-split tori are $\mathrm{H}_{x}(\mathfrak{f})$-conjugate, so, in particular, there is a one-parameter subgroup $\bar{\lambda} \in \mathbf{X}_{*}(\mathrm{~S})$ and an element $\bar{h} \in \mathbf{H}_{x}(\mathfrak{f})$ with $\bar{\lambda}=\bar{h} \bar{\mu}$. Now, let $\lambda \in \mathbf{X}_{*}(\mathbf{S})$ be a lift of $\bar{\lambda}$ and substitute

$\left\{{ }^{\bar{h}} f,{ }^{\bar{h}} h,{ }^{\bar{h}} e\right\}$ for $\{f, h, e\}$. Under the action of $\lambda$ we have the following grading on the Lie algebra $\mathfrak{g}$ :

$$
\mathfrak{g}(i):=\left\{\left.X \in \mathfrak{g}\right|^{\lambda(t)} X=t^{i} \cdot X\right\} \text { and } \overline{\mathfrak{g}}_{x}(i):=\left\{\left.v \in \overline{\mathfrak{g}}_{x}\right|^{\bar{\lambda}(t)} v=t^{i} \cdot v\right\}
$$

For $s \in \mathbb{R}$, we have analogous gradings on $\mathfrak{g}_{x, s}$ and $V_{x, s}$ defined by 


$$
\mathfrak{g}_{x, s}(i):=\left\{\left.Z \in \mathfrak{g}_{x, s}\right|^{\lambda(t)} Z=t^{i} \cdot Z\right\} \text { and } V_{x, s}(i):=\left\{\left.v \in V_{x, s}\right|^{\bar{\lambda}(t)} v=t^{i} \cdot v\right\}
$$

Define $\mathfrak{g}^{1}(i):=\mathfrak{g}^{1} \cap \mathfrak{g}(i), \mathfrak{g}_{x, r}^{1}(i):=\mathfrak{g}_{x, r}^{1} \cap \mathfrak{g}(i)$, and $V_{x, r}^{1}(i):=V_{x, r}^{1} \cap V_{x, r}(i)$.

Lemma IV.12. Suppose Hypothesis IV.3 holds. If $X \in \mathfrak{g}_{x, r}^{1}(2)$ is a lift of e, then, for all $s \in \mathbb{R}$, the map

$$
\operatorname{ad}(X)^{2}: \mathfrak{g}_{x, s-r}^{-1}(-2) \rightarrow \mathfrak{g}_{x, s+r}^{1}(2)
$$

is an isomorphism of $R$-modules.

Proof. By [[12], Lemma 4.3.1], we know that the $\operatorname{map} \operatorname{ad}(X)^{2}: \mathfrak{g}_{x, s-r}(-2) \rightarrow \mathfrak{g}_{x, s+r}(2)$ is an isomorphism of $R$-modules. Thus,

$$
\operatorname{ad}(X)^{2}: \mathfrak{g}_{x, s-r}^{-1}(-2) \rightarrow \mathfrak{g}_{x, s+r}^{1}(2)
$$

is injective.

Let $Z \in \mathfrak{g}_{x, s+r}^{1}(2) \subset \mathfrak{g}_{x, s+r}(2)$. By [[12], 4.3.1], there is an element $Z^{\prime} \in \mathfrak{g}_{x, s-r}(-2)$ such that $\left(\operatorname{ad}(X)^{2}\right)\left(Z^{\prime}\right)=Z$. By Lemma III.9, we may write $Z^{\prime}=Z_{0}^{\prime}+\sum_{i} Z_{i}^{\prime}$, where $Z_{0}^{\prime} \in \mathfrak{h}_{x, s-r}$ and $Z_{i}^{\prime} \in \mathfrak{g}_{x, s-r}^{i}$. By the last line in [[12], Section 4.3], the projection $\mathfrak{g} \rightarrow$ $\mathfrak{g}(i)$ preserves depth, so we let $W$ denote the projection of $Z_{-1}^{\prime} \in \mathfrak{g}$ to $\mathfrak{g}(-2)$. Then, since $X \in \mathfrak{g}_{x, s+r}^{1}(2)$, we have $\left(\operatorname{ad}(X)^{2}\right)(W)=Z$. Thus, the map is surjective.

Corollary IV.13. Suppose Hypotheses IV.3 and IV.7 hold. If $X \in \mathfrak{g}_{x, r}^{1}(2)$ is a lift of $e$, then there are lifts $Y \in \mathfrak{g}_{x,-r}^{-1}$ of $f$ and $H \in \mathfrak{h}_{x, 0}$ of $h$ such that $\{Y, H, X\}$ is a normal $\mathfrak{s l}_{2}(k)$-triple in $\mathfrak{g}$.

Proof. Let $X \in \mathfrak{g}_{x, r}^{1}(2)$ be a lift of $e$. By the previous lemma, $\operatorname{ad}(X)^{2}: \mathfrak{g}_{x,-r}^{-1}(-2) \rightarrow$ $\mathfrak{g}_{x, r}^{1}(2)$ is surjective, so there exists an element $Y \in \mathfrak{g}_{x,-r}^{-1}(-2)$ with $\operatorname{ad}(X)^{2}(Y)=-2 X$. 
By the proof of [[12], Lemma 4.3.1], ad $(e)^{2}: \overline{\mathfrak{g}}_{x}(-2) \rightarrow \overline{\mathfrak{g}}_{x}(2)$ is injective, so since $\operatorname{ad}(e)^{2}(f)=-2 e$ and $\operatorname{ad}(e)^{2}(\bar{Y}-f)=0$, we have that $Y$ is a lift of $f$. Set $H=[X, Y]$. By a computation, $[H, X]=2 X$, so in order to show that $\{Y, H, X\}$ is our desired $\mathfrak{s l}_{2}(k)$-triple, we must verify that $[H, Y]=-2 Y$.

By [[6], Theorem 5.3.2], there exists some $Y^{\prime} \in \mathfrak{g}$ which completes $\{H, X\}$ to an $\mathfrak{s l}_{2}(k)$-triple. By projecting $Y^{\prime}$ to $\mathfrak{g}^{-1}(-2)$, we can assume it lies in this weight space. Now $\operatorname{ad}(X)^{2}\left(Y^{\prime}\right)=\operatorname{ad}(X)^{2}(Y)$, so by Lemma IV.12, since $\operatorname{ad}(X)^{2}$ is injective, we have $Y=Y^{\prime}$.

\subsection{One-parameter subgroups}

We now fix a one-parameter subgroup $\lambda \in \mathbf{X}_{*}^{k}(\mathbf{H})$. The following material is derived from results in [[12], Section 4.4].

Fix an element $X \in \mathcal{N} \cap \mathfrak{g}^{1}$. Suppose Hypothesis IV.9 holds. Then, there exists a normal $\mathfrak{s l}_{2}(k)$-triple $\{Y, H, X\}$ completing $X$ and a homomorphism $\varphi: \mathbf{S L}_{2} \rightarrow \mathbf{G}$ so that $H=d \varphi\left(\left(\begin{array}{cc}1 & 0 \\ 0 & -1\end{array}\right)\right)$ and $Y=d \varphi\left(\left(\begin{array}{ll}0 & 0 \\ 1 & 0\end{array}\right)\right)$. Note that such a map is $\operatorname{Gal}(K / k)$ equivariant.

We will now exhibit a point $y \in \mathcal{B}(H)$ such that $Y \in \mathfrak{g}_{y,-r}^{-1}, H \in \mathfrak{h}_{y, 0}$, and $X \in \mathfrak{g}_{y, r}^{1}$. The argument given in the lemma below (excluding the last paragraph) is due to Gopal Prasad.

Lemma IV.14. (Barbasch and Moy). Suppose Hypothesis IV.9 holds. There exists some $x \in \mathcal{B}(H)$ such that $Y, H, X \in \mathfrak{g}_{x, 0}$.

Proof. (Gopal Prasad) Let $J=\varphi\left(\mathbf{S L}_{2}\left(R_{K}\right)\right) \subset \mathbf{G}^{\circ}(K)$. Then, $B:=(J \rtimes \operatorname{Gal}(K / k))$ is a subgroup of the group of polysimplicial automorphisms of $\mathcal{B}(\mathbf{G}, K)$. Note that $\operatorname{Gal}(K / k)$ is a profinite group; in particular, it is compact and bounded. Thus, $B$ is also bounded, so by [[31], 2.3.1], there exists a fixed point $x^{\prime} \in \mathcal{B}(\mathbf{G}, K)$ under the action of $B$. Let $\mathcal{G}$ denote the smooth affine $R$-group scheme whose $R_{K}$-points 
form the group $\operatorname{stab}_{\mathbf{G}^{\circ}(K)}\left(x^{\prime}\right)$ and whose generic fiber is $\mathbf{G}^{\circ}$. Let $L(\mathcal{G})$ denote the Lie algebra of $\mathcal{G}$, and let $\mathcal{J}$ denote the $R$-group scheme associated to the parahoric subgroup $\mathbf{S L}_{2}\left(R_{K}\right)$. By [[5], 1.7.6], $\varphi$ induces a map of $R_{K}$-schemes from $\mathcal{J}$ to $\mathcal{G}$. Thus $d \varphi\left(\mathfrak{s l}_{2}\left(R_{K}\right)\right) \subset \mathfrak{g}(K)_{x^{\prime}}$. Now, since $x^{\prime}$ is fixed by $\operatorname{Gal}(K / k)$, we have $Y, H, X \in \mathfrak{g}_{x^{\prime}}$.

We have shown that the set of $B$-fixed points $\Omega:=\mathcal{B}(\mathbf{G}, K)^{B}$ is nonempty. Since $\{Y, H, X\}$ is a normal triple, it is $L$-stable, so we have $d \varphi\left(\left(\mathfrak{s l}_{\mathbf{2}}\left(R_{K}\right)\right) \subset \mathfrak{g}(K)_{l\left(x^{\prime}\right)}\right.$, for all $l \in L$. In particular, by [[12], Corollary 4.5.5], we have $l\left(x^{\prime}\right) \in \Omega$, for all $l \in L$, so $\Omega$ is $L$-stable. Thus, since $\Omega$ is convex and closed, and $L$ is a bounded group of isometries, there exists an $L$-fixed point $x \in \Omega$ for which $Y, H, X \in \mathfrak{g}_{x}$.

Under Hypothesis IV.9, there is a homomorphism $\varphi: \mathbf{S L}_{2} \rightarrow \mathbf{G}$ with some nice properties with respect to $\{Y, H, X\}$. Let $\lambda \in \mathbf{X}_{*}^{k}(\mathbf{G})$ be defined by $\lambda(t)=$ $\varphi\left(\left(\begin{array}{cc}t & 0 \\ 0 & t^{-1}\end{array}\right)\right)$.

Definition IV.15. The one-parameter subgroup $\lambda$ described above is said to be adapted to the $\mathfrak{s l}_{2}(k)$-triple $\{Y, H, X\}$.

Remark IV.16. In Section II.1, we declared that an element $X \in \mathfrak{g}^{1}$ is nilpotent provided that there exists some one-parameter subgroup $\mu \in \mathbf{X}_{*}^{k}(\mathbf{G})$ such that

$$
\lim _{t \rightarrow 0}{ }^{(t)} X=0 .
$$

However, assuming Hypothesis IV.9 is valid, we can give an alternate characterization of nilpotence which coincides with this notion. Namely, suppose $X$ lies in $\mathcal{N} \cap \mathfrak{g}^{1}$, and suppose $\{Y, H, X\}$ is a normal $\mathfrak{s l}_{2}(k)$-triple completing $X$. By Jacobson-Morosov, there exists some one-parameter subgroup $\lambda$ for which ${ }^{\lambda(t)} X=t^{2} X$, for $t \in k^{\times}$. Since $\{Y, H, X\}$ is normal, $H$ is $L$-fixed; in particular, we may assume $\lambda$ is fixed by $L$. Thus, under Hypothesis IV.9, $X$ lies in $\mathcal{N} \cap \mathfrak{g}^{1}$ if and only if there exists some one-parameter 
subgroup in $\mathbf{X}_{*}^{k}(\mathbf{H})$ which annihilates $X$ in the limit described above.

As noted in the remark above, if $\{Y, H, X\}$ is normal, we may assume $\lambda \in \mathbf{X}_{*}^{k}(\mathbf{H})$. Define $M=C_{\mathbf{G}^{\circ}(k)}(\lambda)$.

Corollary IV.17. Suppose Hypotheses IV.7 and IV.9 hold. There exists some $y \in$ $\mathcal{B}(H)$ such that $Y \in \mathfrak{g}_{y,-r}^{-1}, X \in \mathfrak{g}_{y, r}^{1}$, and $H \in \mathfrak{h}_{y, 0}$.

Proof. Together, Hypotheses IV.7 and IV.9 imply that the residue field $\mathfrak{f}$ has cardinality greater than 3. By Lemma IV.14, there is an element $x \in \mathcal{B}(H)$ such that $Y, H, X \in \mathfrak{g}_{x}$. Since $\lambda\left(R^{\times}\right) \subset J$ as in the proof of Lemma IV.14, we know that the point $x$ is fixed by $\lambda\left(R^{\times}\right)$. In particular, by [[12], Corollary 4.4.2], $x$ lies in $\mathcal{B}(M)$. Choose an apartment $\mathcal{A} \subset \mathcal{B}(H)$ which contains $x$. Since $\lambda$ lies in the center of $M, \lambda$ acts on every apartment in $\mathcal{B}(M)$ by translation. Using this fact, define $y=x+\frac{r}{2} \cdot \lambda \in \mathcal{A}$. By Lemma IV.14, $X \in \mathfrak{g}_{x, 0}^{1}$, so we write $X=\sum_{\psi} X_{\psi}$, where $X_{\psi} \in \mathfrak{g}_{\psi}$, for $\psi(x) \geq 0$. For all such $\psi$ such that $X_{\psi} \neq 0$, we have $\langle\lambda, \dot{\psi}\rangle=2$ since $\lambda$ acts by squares on $X$ by Hypothesis IV.3. For any such $\psi$, we have

$$
\psi(y)=\psi(x)+\frac{r}{2}\langle\lambda, \dot{\psi}\rangle \geq r
$$

Therefore, $X$ lies in $\mathfrak{g}_{y, r}^{1}$. By a similar argument, $H \in \mathfrak{h}_{y, 0}$ and $Y \in \mathfrak{g}_{y,-r}^{-1}$. 


\section{CHAPTER V}

\section{The main result: a parametrization of nilpotent orbits in the Lie algebra of a reductive $p$-adic group}

In this chapter, we assume that $L$ is a finite cyclic group of order $m$.

Fix $r \in \mathbb{R}$. We now discuss the notion of the building set associated to an $\mathfrak{s l}_{2}(k)$ triple, so we assume that Hypotheses IV.7 and IV.9 hold. We follow the discussion in [[12], Section 5]. Fix $Z \in \mathcal{N}^{1}$ and $s \in \mathbb{R}$.

\subsection{The building set}

\section{Definition V.1.}

$$
\mathcal{B}(Z, s):=\left\{z \in \mathcal{B}(G) \mid Z \in \mathfrak{g}_{z, s}\right\} .
$$

From [[12]], we know that $\mathcal{B}(Z, s)$ is nonempty, convex and closed.

\section{Definition V.2.}

$$
\mathcal{B}_{L}(Z, s):=\mathcal{B}(Z, s) \cap \mathcal{B}(H) .
$$

Corollary IV.17 tells us that when $Z \in \mathcal{N}$ and the hypothesis in Section IV.1 is satisfied, the set $\mathcal{B}_{L}(Z, s)$ is nonempty. From Definition V.2, we see that if $x \in$ $\mathcal{B}_{L}(Z, s)$, then $F_{L}^{*}(x) \subset \mathcal{B}_{L}(Z, s)$. In particular, $\mathcal{B}_{L}(Z, s)$ is the union of generalized $(s, L)$-facets of $\mathcal{B}(H)$. Since $\mathcal{B}(Z, s)$ and $\mathcal{B}(H)$ are convex, $\mathcal{B}_{L}(Z, s)$ is also convex. 
Lemma V.3. $\mathcal{B}_{L}(Z, s)$ is closed.

Proof. This follows from the fact that $\mathcal{B}(H)$ and $\mathcal{B}(Z, s)$ are closed.

Fix a (possibly trivial) normal $\mathfrak{s l}_{2}(k)$-triple $\{Y, H, X\}$ in $\mathfrak{g}$.

Definition V.4. Define

$$
\mathcal{B}(Y, H, X):=\mathcal{B}(X, r) \cap \mathcal{B}(Y,-r) .
$$

Definition V.5. Define

$$
\mathcal{B}_{L}(Y, H, X):=\mathcal{B}(Y, H, X)^{L}
$$

By [[12], Remark 5.1.5], $\mathcal{B}(Y, H, X)$ is convex. In particular, $\mathcal{B}_{L}(Y, H, X)$ is a closed, convex set which is the union of generalized $(r, L)$-facets.

Lemma V.6. Suppose $F_{1, L}^{*}, F_{2, L}^{*}$ are maximal generalized $(r, L)$-facets in $\mathcal{B}_{L}(Y, H, X)$. Then, $F_{1, L}^{*}$ and $F_{2, L}^{*}$ are strongly $r$-associated.

Proof. Let $x_{i} \in F_{i, L}^{*}$, for $i=1,2$, and let $\mathcal{A}$ be an apartment of $\mathcal{B}(H)$ containing $x_{1}$ and $x_{2}$. If $x_{1} \notin A\left(\mathcal{A}, F_{2, L}^{*}\right)$, then, since $\mathcal{B}_{L}(Y, H, X)$ is convex, there is some generalized $(r, L)$-facet in $\mathcal{B}_{L}(Y, H, X)$ of strictly larger dimension than $F_{2, L}^{*} \cap \mathcal{A}$, a contradiction. Thus, $A\left(\mathcal{A}, F_{1, L}^{*}\right) \subset A\left(\mathcal{A}, F_{2, L}^{*}\right)$, and similarly, $A\left(\mathcal{A}, F_{2, L}^{*}\right) \subset A\left(\mathcal{A}, F_{1, L}^{*}\right)$.

We now suppose that Hypotheses IV.7, IV.9, and IV.11 hold. Fix $X \in \mathcal{N}^{1} \backslash\{0\}$ and $r \in \mathbb{R}$. Suppose that $\{Y, H, X\}$ is a normal $\mathfrak{s l}_{2}(k)$-triple completing $X$ and that $\lambda \in \mathbf{X}_{*}^{k}(\mathbf{H})$ is adapted to $\{Y, H, X\}$. Fix $x \in \mathcal{B}_{L}(Y, H, X)$. We would like for ${ }^{H} X$ to be the unique nilpotent $H$-orbit in $\mathfrak{g}^{1}$ of minimal dimension which intersects the coset $X+\mathfrak{g}_{x, r^{+}}^{1}$ nontrivially. The next lemma gives us a decomposition of the coset $X+\mathfrak{g}_{x, r^{+}}^{1}$ up to conjugacy by $H_{x}^{+}$. Recall that the one-parameter subgroup $\lambda$ induces 
a grading on the Lie algebra of $\mathfrak{g}$ as noted in the beginning of Section IV.1.1. For the following lemma, we imitate the argument in [[12], 5.2.1].

Lemma V.7. Assume Hypotheses IV.7, IV.9, and IV.11 hold. Then

$$
H_{x}^{+}\left(X+C_{\mathfrak{g}_{x, r^{+}}^{1}}(Y)\right)=X+\mathfrak{g}_{x, r^{+}}^{1}
$$

Proof. " $\subset$ ": By [[1], Prop 1.4.3], $H_{x}^{+}$induces the trivial action on $V_{x, r}^{1}$.

" $\supset$ ": From Hypothesis IV.3, we know that as a representation of $\langle Y, H, X\rangle, \mathfrak{g}$ decomposes into a direct sum of irreducible $\langle Y, H, X\rangle$-modules with highest weight at most $p-3$. Thus, we can write

$$
\mathfrak{g}=\bigoplus_{\rho \in \mathbb{Z}_{\geq 0}} \mathfrak{g}_{\rho}
$$

where $\mathfrak{g}_{\rho}$ is the isotypic component of $\langle Y, H, X\rangle$-modules in $\mathfrak{g}$ with highest weight $\rho$. In other words, $\mathfrak{g}_{\rho}$ is the direct sum of irreducible $\langle Y, H, X\rangle$-submodules of $\mathfrak{g}$ of dimension $\rho+1$. Let $\mathfrak{g}(\rho, i):=\mathfrak{g}_{\rho} \cap \mathfrak{g}(i)$, and let $\mathfrak{g}^{1}(\rho, i):=\mathfrak{g}(\rho, i) \cap \mathfrak{g}^{1}$. Then, $\mathfrak{g}(i)=\oplus_{\rho} \mathfrak{g}(\rho, i)$ and thus $\mathfrak{g}=\oplus_{\rho, i} \mathfrak{g}(\rho, i)$. Also, note that we have that $C_{\mathfrak{g}}(X)=\oplus_{i \geq 0} \mathfrak{g}(i, i)$ since $\operatorname{ad}(X)(\mathfrak{g}(i))=\mathfrak{g}(i+2)$, and $\mathfrak{g}(i, i)$ is the sum of $i$-weight spaces of all irreducible $\langle Y, H, X\rangle$-submodules with highest weight $i$. Similarly, we have $C_{\mathfrak{g}}(Y)=\oplus_{i \geq 0} \mathfrak{g}(i,-i)$. It follows that $C_{\mathfrak{g}^{1}}(Y)=\oplus_{i \geq 0} \mathfrak{g}^{1}(i,-i)$ and $C_{\mathfrak{g}^{1}}(X)=\oplus_{i \geq 0} \mathfrak{g}^{1}(i, i)$. Define $\mathfrak{g}_{x, s}(\rho, i):=$ $\mathfrak{g}_{x, s} \cap \mathfrak{g}(\rho, i)$. By the proof of [[12], Lemma 5.2.1], since the projection $\mathfrak{g} \rightarrow \mathfrak{g}(i)$ preserves depth, we have the following decompositions:

$$
\mathfrak{g}_{x, s}(i)=\bigoplus_{\rho \in \mathbb{Z}_{\geq 0}} \mathfrak{g}_{x, s}(\rho, i)
$$

and

$$
\mathfrak{g}_{x, s}=\bigoplus_{\rho, i} \mathfrak{g}_{x, s}(\rho, i)
$$


From the first decomposition, using Proposition III.9 it follows that

$$
\mathfrak{h}_{x, s}(i)=\bigoplus_{\rho \in \mathbb{Z}_{\geq 0}} \mathfrak{h}_{x, s}(\rho, i)
$$

and

$$
\mathfrak{g}_{x, s}^{1}(i)=\bigoplus_{\rho \in \mathbb{Z}_{\geq 0}} \mathfrak{g}_{x, s}^{1}(\rho, i)
$$

We claim that the following decompositions hold:

$$
(\dagger) \text { For } i>0, \mathfrak{g}_{x, s}^{1}(i)=\operatorname{ad}(X)\left(\mathfrak{h}_{x, s-r}(i-2)\right)
$$

and

$$
(\dagger \dagger) \text { For } i \leq 0, \mathfrak{g}_{x, s}^{1}(i)=\mathfrak{g}_{x, s}^{1}(-i, i)+\operatorname{ad}(X)\left(\mathfrak{h}_{x, s-r}(i-2)\right)
$$

The first decomposition $(\dagger)$ is obtained using decompositions $\mathfrak{h}_{x, s}(i)=\bigoplus_{\rho \in \mathbb{Z}_{\geq 0}} \mathfrak{h}_{x, s}(\rho, i)$ and $\mathfrak{g}_{x, s}^{1}(i)=\bigoplus_{\rho \in \mathbb{Z}_{\geq 0}} \mathfrak{g}_{x, s}^{1}(\rho, i)$ and the fact that $\mathfrak{g}^{1}(\rho, i)=\left\{Z \in \mathfrak{g}^{1}(i) \mid(\operatorname{ad}(X) \circ\right.$ $\operatorname{ad}(Y))(Z)=j(\rho, i) \cdot Z\}$, which can be found in [[12], 5.2.1].

The second decomposition $(\dagger \dagger)$ results from the fact that for $Z \in \mathfrak{g}_{x, s}^{1}(i), i \leq 0, Z$ may lie in $C_{\mathfrak{g}^{1}}(Y)$. We also use the fact that $\mathfrak{g}_{x, s}^{1}(i)=\bigoplus_{\rho \in \mathbb{Z}_{\geq 0}} \mathfrak{g}_{x, s}^{1}(\rho, i)$.

Now, summing over all $i$, we obtain

$$
(\star) \mathfrak{g}_{x, s}^{1}=C_{\mathfrak{g}_{x, s}^{1}}(Y)+\operatorname{ad}(X)\left(\mathfrak{h}_{x, s-r}\right)
$$

Let $Z \in \mathfrak{g}_{x, r^{+}}^{1}$. We will show the existence of elements $h \in H_{x}^{+}$and $C \in C_{\mathfrak{g}_{x, r^{+}}^{1}}(Y)$ such that ${ }^{h}(X+C)=X+Z$. First, let $h_{0}=1$ and $C_{0}=0$. Now, choose $s_{1} \in \mathbb{R}$ with $\mathfrak{g}_{x, r^{+}}^{1}=\mathfrak{g}_{x, s_{1}}^{1} \neq \mathfrak{g}_{x, s_{1}^{+}}^{1}$. Using $(\star)$, we can write $Z=C_{1}^{\prime}+\operatorname{ad}(X)\left(P_{1}\right)$ where $C_{1}^{\prime} \in C_{\mathfrak{g}_{x, s_{1}}^{1}}(Y)$ and $P_{1} \in \mathfrak{h}_{x,\left(s_{1}-r\right)}$. Applying Hypothesis IV.11 with $s=s_{1}-r$ and $t=s_{1}$, there exists a map $\phi_{x}: \mathfrak{h}_{x, s_{1}-r} \rightarrow H_{x, s_{1}-r}$ such that 


$$
(1)^{\phi_{x}\left(-P_{1}\right)}\left(X+C_{0}+C_{1}^{\prime}\right)=X+C_{1}^{\prime}+\operatorname{ad}(X)\left(P_{1}\right) \bmod \mathfrak{g}_{x, s_{1}^{+}}^{1}
$$

Set $h_{1}^{\prime}=\phi_{x}\left(-P_{1}\right)$. Rewriting the above equation, we have $h_{1}^{\prime} h_{0}\left(X+C_{0}+C_{1}^{\prime}\right)=$ $X+Z-Z_{1}$ for some $Z_{1} \in \mathfrak{g}_{x, s_{1}^{+}}^{1}$. Let $h_{1}=h_{1}^{\prime} h_{0}$ and $C_{1}=C_{0}+C_{1}^{\prime}$. Now, fix an element $s_{2}>s_{1}$ such that $\mathfrak{g}_{x, s_{1}^{+}}^{1}=\mathfrak{g}_{x, s_{2}}^{1} \neq \mathfrak{g}_{x, s_{2}^{+}}^{1}$. Continuing as in the previous case, from $(\star)$, we write $Z_{1}=C_{2}^{\prime}+\operatorname{ad}(X)\left(P_{2}\right)$ where $C_{2}^{\prime} \in C_{\mathfrak{g}_{x, s_{2}}^{1}}(Y)$ and $P_{2} \in \mathfrak{h}_{x, s_{2}-r}$. Applying Hypothesis IV.11 and (1), there exists a map $\phi_{x}$ such that

$$
\begin{aligned}
\phi_{x}\left(-P_{2}\right)\left(X+C_{1}+C_{2}^{\prime}\right) & ={ }_{2}^{\prime}\left(X+Z-Z_{1}+C_{2}^{\prime}\right) \bmod \mathfrak{g}_{x, s_{2}^{+}}^{1} \\
& =X+Z-Z_{1}+C_{2}^{\prime}+\operatorname{ad}(X) P_{2} \bmod \mathfrak{g}_{x, s_{2}^{+}}^{1} \\
& =X+Z-Z_{2} .
\end{aligned}
$$

where $Z_{2} \in \mathfrak{g}_{x, s_{2}^{+}}^{1}$ and $h_{2}^{\prime}=\phi_{x}\left(-P_{2}\right)$. Set $h_{2}=h_{2}^{\prime} h_{1}$ and $C_{2}=C_{1}+C_{2}^{\prime}$. Proceeding as above, we obtain a strictly increasing sequence $\left\{s_{i}\right\}$ with $s_{1}>r$ such that $h_{n} \in H_{x}^{+}$ and $h_{n}^{\prime} \in H_{x,\left(s_{n}-r\right)}$. Moreover, we have elements $C_{n}=C_{n-1}+C_{n}^{\prime} \in C_{\mathfrak{g}_{x, r}^{1}}(Y)$ such that $C_{n}^{\prime} \in C_{\mathfrak{g}_{x, s_{n}}^{1}}(Y)$ and

$$
h_{n}\left(X+C_{n}\right)=X+Z \bmod \mathfrak{g}_{x, s_{n}^{+}}^{1}
$$

Now set $h=\lim _{n \rightarrow \infty} h_{n}$ and $C=\lim _{n \rightarrow \infty} C_{n}$. Clearly, these elements lie in $H_{x}^{+}$and $C_{\mathfrak{g}_{x, r^{+}}^{1}}(Y)$, respectively. By construction, we have ${ }^{h}(X+C)=X+Z$.

Lemma V.8. Suppose Hypothesis IV.9 holds. Then

$$
\left(X+C_{\mathfrak{g}^{1}}(Y)\right) \cap{ }^{H} X=\{X\} .
$$

Proof. The result [ [34], V.7 (9)] tells us that $\left.\left(X+C_{\mathfrak{g}}(Y)\right)\right) \cap{ }^{G} X=\{X\}$, so, consequently, $\left(X+C_{\mathfrak{g}^{1}}(Y)\right) \cap^{H} X \subset\{X\}$. Since $\{X\}$ is clearly contained in the intersection, the result follows. 
Corollary V.9. Suppose Hypotheses IV.7, IV.9, and IV.11 hold. Then

$$
\left(X+\mathfrak{g}_{x, r^{+}}^{1}\right) \cap{ }^{H} X={ }^{H_{x}^{+}} X .
$$

Proof. " $\supset$ ": This inclusion follows from the first part of the proof of Lemma V.7. " $\subset$ ": Using Lemma V.7, we have $X+\mathfrak{g}_{x, r^{+}}^{1}=H_{x}^{+}\left(X+C_{\mathfrak{g}_{x, r^{+}}^{1}}(Y)\right)$. Thus, if $Z$ lies in $\left(X+\mathfrak{g}_{x, r^{+}}^{1}\right) \cap{ }^{H} X$, then there exist elements $h_{1} \in H_{x}^{+}, h_{2} \in H$ and $Z_{1} \in C_{\mathfrak{g}_{x, r^{+}}^{1}}(Y)$ such that $Z={ }^{h_{1}}\left(X+Z_{1}\right)={ }^{h_{2}} X$. Thus ${ }_{1}^{-1} Z=X+Z_{1}={ }_{1}^{h_{1}^{-1}} h_{2} X$, so by Corollary V.8, we have

$$
X+Z_{1} \in\left(X+C_{\mathfrak{g}^{1}}(Y)\right) \cap{ }^{H} X=\{X\}
$$

Thus, $Z_{1}=0$.

Definition V.10. We denote by $\mathcal{O}_{1}(0)$ the set of all nilpotent $H$-orbits in $\mathfrak{g}^{1}$.

In the statement of the following corollary, if $\mathcal{O}_{1}$ is an element of $\mathcal{O}_{1}(0)$, we let $\overline{\mathcal{O}_{1}}$ denote the $p$-adic closure of $\mathcal{O}_{1}$.

Corollary V.11. Suppose Hypotheses IV.7, IV.9, and IV.11 hold. If $\mathcal{O}_{1} \in \mathcal{O}_{1}(0)$ such that

$$
\left(X+\mathfrak{g}_{x, r^{+}}^{1}\right) \cap \mathcal{O}_{1} \neq \emptyset
$$

then ${ }^{H} X \subset \overline{\mathcal{O}_{1}}$.

Proof. (J.L. Waldspurger) Let $Z \in\left(X+\mathfrak{g}_{x, r^{+}}^{1}\right) \cap \mathcal{O}_{1}$. Then by Lemma V.7, there exist elements $h \in H_{x}^{+}$and $C \in C_{\mathfrak{g}_{x, r^{+}}^{1}}(Y)$ such that ${ }^{h}(X+C)=Z \in \mathcal{O}_{1}$. Inverting $h$, we have $X+C \in \mathcal{O}_{1}$, which is therefore nilpotent. By Jacobson-Morosov, there exists a one-parameter subgroup $\mu \in \mathbf{X}_{*}^{k}(\mathbf{H})$ such that ${ }^{\mu(t)}(X+C)=t^{2} \cdot(X+C)$ for all 
$t \in k^{\times}$. Recall that we let $\lambda$ denote the one-parameter subgroup adapted to $\mathfrak{s l}_{2}(k)$ triple $\{Y, H, X\}$. In particular, ${ }^{\lambda(t)} X=t^{2} \cdot X$, for $t \in k^{\times}$. Moreover, from the proof of Lemma V.7, we know that $C_{\mathfrak{g}^{1}}(Y)=\oplus_{i \geq 0} \mathfrak{g}^{1}(i,-i)$, so, in particular, $C \in \oplus_{i \leq 0} \mathfrak{g}^{1}(i)$. Thus,

$$
\lim _{t \rightarrow 0} \lambda^{(t)^{-1} \mu(t)}(X+C)=\lim _{t \rightarrow 0}^{\lambda(t)^{-1}} t^{2}(X+C)=X+\lim _{t \rightarrow 0}^{\lambda(t)^{-1}} C=X
$$

Corollary V.12. Suppose Hypotheses IV.7, IV.9, and IV.11 hold. Choose $F_{L}^{*} \in$ $\mathcal{F}_{L}(r)$ such that $F_{L}^{*} \subset \mathcal{B}_{L}(Y, H, X)$. If $\mathcal{O}_{1} \in \mathcal{O}_{1}(0)$ such that

$$
\left(X+\mathfrak{g}_{F_{L}^{*}}^{1,+}\right) \cap \mathcal{O}_{1} \neq \emptyset
$$

then ${ }^{H} X \subset \overline{\mathcal{O}_{1}}$.

Proof. If $x \in F_{L}^{*}$, then $X \in \mathfrak{g}_{x, r}^{1}=\mathfrak{g}_{F_{L}^{*}}^{1}$. Thus, by Corollary V.11, ${ }^{H} X \subset \overline{\mathcal{O}_{1}}$.

Definition V.13. Define $I_{r}^{n}:=\left\{\left(F_{L}^{*}, v\right) \in I_{r} \mid v\right.$ is degenerate in $\left.V_{F_{L}^{*}}^{1}\right\}$.

Suppose $\left(F_{L}^{*}, e\right) \in I_{r}^{n}$. Let $x \in F_{L}^{*}$. If $e$ is trivial, then we call the normal $\mathfrak{s l}_{2}(\mathfrak{f})$ triple completing $e\left(\right.$ in $\left.V_{x,-r}^{-1} \times V_{x, 0}^{0} \times V_{x, r}^{1}\right)$ the trivial $\mathfrak{s l}_{2}(\mathfrak{f})$-triple. Similarly, given a trivial $\mathfrak{s l}_{2}(\mathfrak{f})$-triple, we declare that the $\mathfrak{s l}_{2}(k)$-triple lifting our $\mathfrak{s l}_{2}(\mathfrak{f})$-triple is the trivial $\mathfrak{s l}_{2}(k)$-triple.

Lemma V.14. Suppose all hypotheses from Section IV.1 hold, and let $\left(F_{L}^{*}, e\right) \in I_{r}^{n}$.

1. Fix $x \in F_{L}^{*}$. There exists a normal $\mathfrak{s l}_{2}(\mathfrak{f})$-triple $(f, h, e) \in V_{x,-r}^{-1} \times V_{x, 0}^{0} \times V_{x, r}^{1}$ completing e and a normal $\mathfrak{s l}_{2}(k)$-triple $\{Y, H, X\}$ which lifts $\{f, h, e\}$. 
2. For any $x \in F_{L}^{*}$, for any normal $\mathfrak{s l}_{2}(\mathfrak{f})$-triple $(f, h, e) \in V_{x,-r}^{-1} \times V_{x, 0}^{0} \times V_{x, r}^{1}$ completing e, and for any normal $\mathfrak{s l}_{2}(k)$-triple $\{Y, H, X\}$ which lifts $\{f, h, e\}$, we have $F_{L}^{*} \subset \mathcal{B}_{L}(Y, H, X)$, and ${ }^{H} X$ is the unique nilpotent $H$-orbit in $\mathfrak{g}^{1}$ of minimal dimension which intersects the coset e nontrivially.

Proof. If $e$ is trivial, then all the conclusions are obvious. Assume $e$ is nontrivial. By Hypothesis IV.3, there exist elements $H \in \mathfrak{h}_{x, 0}$ and $Y \in \mathfrak{g}_{x,-r}^{-1}$ such that the image $\{f, h, e\}$ of the triple $\{Y, H, X\}$ forms a normal $\mathfrak{s l}_{2}(\mathfrak{f})$-triple in $\overline{\mathfrak{g}}_{x}$. By Corollary IV.13, there is a normal $\mathfrak{s l}_{2}(k)$-triple lifting $\{f, h, e\}$.

For (2), suppose $x \in F_{L}^{*}$, the triple $\{f, h, e\}\left(\subset V_{x,-r}^{-1} \times V_{x, 0}^{0} \times V_{x, r}^{1}\right)$ is a normal $\mathfrak{s l}_{2}(\mathfrak{f})$-triple in $\overline{\mathfrak{g}}_{x}$ completing $e$, and $\{Y, H, X\}$ is a normal $\mathfrak{s l}_{2}(k)$-triple which lifts $\{f, h, e\}$. Note that since $x \in F_{L}^{*}$, we have $F_{L}^{*} \subset \mathcal{B}_{L}(Y, H, X)$ since $X \in \mathfrak{g}_{x, r}^{1}$ and $Y \in \mathfrak{g}_{x,-r}^{-1}$. Now, if ${ }^{H} Z$ is a nilpotent $H$-orbit such that $\left(X+\mathfrak{g}_{x, r^{+}}^{1}\right) \cap{ }^{H} Z \neq \emptyset$, then by Corollary V.12, we have ${ }^{H} X \subset \overline{{ }^{H} Z}$. Thus, since the closure of any $H$-orbit is the union of the the orbit itself and orbits of strictly smaller dimension, ${ }^{H} X$ is the unique nilpotent $H$-orbit in $\mathfrak{g}^{1}$ of minimal dimension which intersects the coset $e$ nontrivially.

Definition V.15. Suppose all the hypotheses of Section IV.1 hold. For $\left(F_{L}^{*}, e\right) \in$ $I_{r}^{n}$, let $\mathcal{O}_{1}\left(F_{L}^{*}, e\right)$ denote the unique nilpotent $H$-orbit of minimal dimension which intersects the coset $e$ nontrivially.

Remark V.16. If $h \in H$ and $\left(F_{L}^{*}, e\right) \in I_{r}^{n}$, then it is clear from Definition V.15 that $\mathcal{O}_{1}\left(h F_{L}^{*},{ }^{h} e\right)=\mathcal{O}_{1}\left(F_{L}^{*}, e\right)$.

Lemma V.17. Suppose all the hypotheses of Section IV.1 hold. The map $\varphi: I_{r}^{n} \rightarrow$ $\mathcal{O}_{1}(0)$ defined by $\left(F_{L}^{*}, e\right) \mapsto \mathcal{O}_{1}\left(F_{L}^{*}, e\right)$ induces a well-defined map from $I_{r}^{n} / \sim$ to $\mathcal{O}_{1}(0)$.

Proof. Suppose $\left(F_{i, L}^{*}, e_{i}\right) \in I_{r}^{n}$ and $\left(F_{1, L}^{*}, e_{1}\right) \sim\left(F_{2, L}^{*}, e_{2}\right)$. Suppose $e_{i} \in V_{F_{i, L}^{*}}^{1}$ is nontrivial. Choose $x_{i} \in C\left(F_{i, L}^{*}\right)$. Since $\left(F_{1, L}^{*}, e_{1}\right) \sim\left(F_{2, L}^{*}, e_{2}\right)$, there exists an element $h \in H$ and an apartment $\mathcal{A} \subset \mathcal{B}(H)$ such that 
1. $A\left(\mathcal{A}, F_{1, L}^{*}\right)=A\left(\mathcal{A}, h F_{2, L}^{*}\right) \neq \emptyset$,

2. $e_{1}={ }^{h} e_{2}$ in $V_{F_{1, L}^{*}}^{1}=V_{h F_{2, L}^{*}}^{1}$.

As a result of Remark V.16, we assume now that $h=1$. Let $\mathbf{S}$ be the maximal $k$-split torus of $\mathbf{H}$ corresponding to the apartment $\mathcal{A}$. Let $\mathbf{S}$ denote the maximal $\mathfrak{f}$-split torus inside $\mathrm{H}_{x_{1}}$ corresponding to $\mathbf{S}$. By the previous lemma, we can complete $e_{1}$ to a normal $\mathfrak{s l}_{2}(\mathfrak{f})$-triple $\left(f, h, e_{1}\right) \in V_{x_{1}, r}^{-1} \times V_{x_{1}, 0}^{0} \times V_{x_{1}, r}^{1}$. Suppose that $\bar{\lambda} \in \mathbf{X}_{*}^{\mathfrak{f}}\left(\mathrm{H}_{x_{1}}\right)$ is adapted to this triple. Since any one-parameter subgroup is contained in some maximal $\mathfrak{f}$-split torus, there exists some $h^{\prime} \in H_{x_{1}}$ such that $\bar{h}^{\prime} \bar{\lambda} \in \mathbf{X}_{*}^{\mathfrak{f}}(\mathrm{S})$. By Lemma III.43, there is an element $h^{\prime \prime} \in H_{x_{1}} \cap H_{x_{2}}$ such that its image in $\operatorname{Aut}_{\mathfrak{f}}\left(V_{F_{i, L}^{*}}^{1}\right)$ coincides with the image of $h^{\prime}$. In other words, we have $\left.\operatorname{Ad}\left(h^{\prime \prime}\right)\right|_{V_{F_{i, L}^{*}}^{1}}=\left.\operatorname{Ad}\left(h^{\prime}\right)\right|_{V_{F_{i, L}^{*}}^{1}}$. In summary, we have

$$
{ }^{h^{\prime}} e_{1}={ }^{h^{\prime \prime}} e_{1}={ }^{h^{\prime \prime}} e_{2} \text { in } V_{F_{1, L}^{*}}^{1}=V_{F_{1, L}^{*}}^{1}=V_{F_{2, L}^{*}}^{1} .
$$

Now let $\lambda \in \mathbf{X}_{*}^{k}(\mathbf{S})$ be a lift of ${ }^{\overline{h^{\prime}}} \bar{\lambda}$. As usual, we have a grading of $\mathfrak{g}$ under the action of $\lambda$. As in the proof of Lemma V.7, we also have the decomposition

$$
\mathfrak{g}_{F_{i, L}^{*}}=\bigoplus_{j} \mathfrak{g}_{F_{i, L}^{*}}(j)
$$

By Lemma III.40, there is a lift $X \in \mathfrak{g}_{F_{1, L}^{*}}^{1}(2) \cap \mathfrak{g}_{F_{2, L}^{*}}^{1}(2)$ of ${ }^{h^{\prime \prime}} e_{i}$. Note that $X$ lifts ${ }^{h^{\prime}} e_{i}$ and ${ }^{h^{\prime \prime-1}} X$ lifts $e_{i}$. We apply Corollary IV.13 and Lemma V.14 to conclude

$$
\mathcal{O}_{1}\left(F_{i, L}^{*}, e_{i}\right)=\mathcal{O}_{1}\left(F_{i, L}^{*},{ }^{h^{\prime \prime}} e_{i}\right)={ }^{H} X
$$

\section{$5.2 \quad$ Noticed orbits}

Now, in order to obtain a bijection between depth $r$ cosets and nilpotent $H$-orbits, we have to shrink $I_{r}^{n} / \sim$. We do this by restricting to noticed orbits in $\mathfrak{g}_{F_{L}^{*}}^{1} / \mathfrak{g}_{F_{L}^{*}}^{1,+}$. 
Definition V.18. Define $I_{r}^{d} \subset I_{r}^{n}$ to be those pairs $\left(F_{L}^{*}, e\right) \in I_{r}^{n}$ such that for any $x \in F_{L}^{*}$, for any normal $\mathfrak{s l}_{2}(\mathfrak{f})$-triple $(f, h, e) \in V_{x,-r} \times V_{x, 0} \times V_{x, r}$ completing $e$, and for any normal $\mathfrak{s l}_{2}(k)$-triple $\{Y, H, X\}$ in $\mathfrak{g}$ which lifts $\{f, h, e\}$, we have that $F_{L}^{*}$ is a maximal generalized $(r, L)$-facet in $\mathcal{B}_{L}(Y, H, X)$.

In order to simplify notation below, we will refer to a generalized $(0, L)$-facet as a generalized $L$-facet.

Remark V.19. In the case when $r=0$ and $|L|=2$, we can give an alternate characterization of noticed nilpotent orbits inside a Lie algebra which is analogous to the definition in [[22], Definition 2.1]. In particular, if a pair $\left(F_{L}^{*}, e\right)$ lies in $I_{0}^{d}$, then $C_{V_{F_{L}^{*}}^{L}}(e)$ does not contain certain non-central semisimple elements. We give a precise formulation below which connects the notions of the building set of a triple with the notion of a noticed orbit in [[22], Definition 2.1] which is encountered in the case of symmetric spaces of real reductive groups.

\subsubsection{Conjugacy of triples over $\mathfrak{f}$}

Suppose $\left(F_{L}^{*}, e\right) \in I_{0}^{n}$, and let $x \in F_{L}^{*}$. In this paragraph, we let $\mathrm{L}_{x}$ denote the Lie algebra of $\mathrm{G}_{x}$. Suppose $\{f, h, e\}$ and $\left\{f^{\prime}, h^{\prime}, e\right\}$ are $\mathfrak{s l}_{2}(\mathfrak{f})$-triples in $\mathrm{L}_{x}$ which complete e.

Under some restrictions on the characteristic of $\mathfrak{f}$, it is shown in [[6], Proposition 5.7.4] that if $\{f, h, e\}$ is a normal $\mathfrak{s l}_{2}(\mathfrak{f})$-triple in $\mathrm{L}_{x}$ completing $e$, then $C_{\mathrm{L}_{x}}(e)$ is a subalgebra of $\mathrm{L}_{x}$ of the form $\mathfrak{c} \oplus \mathfrak{u}$, where $\mathfrak{c}$ is a reductive subalgebra which centralizes the triple $\{f, h, e\}$ and $\mathfrak{u}$ is a nilpotent ideal in $C_{\mathrm{L}_{x}}(e)$. In particular, the Lie algebra of any $\mathfrak{f}$-split torus of $C_{\mathrm{G}_{x}}(e)$ lies in $\mathfrak{c}$, for any choice of an $\mathfrak{s l}_{2}(\mathfrak{f})$-triple completing $e$. Accordingly, we consider the $\mathfrak{f}$-rank of $\mathfrak{c}$ in the sense of [[3], 21.1].

Note that since $\{f, h, e\}$ is normal, the subalgebra it generates (in $\mathrm{L}_{x}$ ) is $L$-stable, so both $C_{\mathrm{G}_{x}}(e)$ and $\mathfrak{c}$ are $L$-stable. Thus, the $\mathfrak{f}$-rank of $C_{\mathbf{H}_{x}}(e)$ is the $\mathfrak{f}$-rank of $\mathfrak{c}^{L}$. 


\subsubsection{An equivalent notion of a noticed pair}

For the next proposition only, we assume that $|L|=2$. Under these assumptions, as mentioned in Section II, Helminck-Wang show that every maximal $k$-split torus of $\mathbf{H}$ lies in an $L$-stable maximal $k$-split torus in $\mathbf{G}$.

In the following proposition, we take the $\mathfrak{f}$-rank of $C_{\mathrm{L}_{x}}(e)$ to mean the $\mathfrak{f}$-rank of the centralizer $\mathfrak{c}:=C_{\mathrm{L}_{x}}($ im $\phi)$, where $\phi: \mathfrak{s l}_{2}(\mathfrak{f}) \rightarrow V_{F_{L}^{*}}$ is an $\mathfrak{f}$-map whose image contains $e$.

Proposition V.20. Let $\left(F_{L}^{*}, e\right) \in I_{0}^{n}$. Suppose Hypothesis IV.3 holds, $\mathfrak{f}$ is finite, $|L|=2$, and $p>3(j-1)$, where $j$ is the Coxeter number as defined in [[6], Section 1.9]. The following are equivalent:

1. $C_{L_{x}^{L}}(e) \cap\left[L_{x}, L_{x}\right]$ has $\mathfrak{f}$-rank equal to zero.

2. $\left(F_{L}^{*}, e\right)$ lies in $I_{0}^{d}$.

Proof. (1) $\Rightarrow(2)$ : Suppose there is some $x \in F_{L}^{*}$ for which there exists a lift $\{Y, H, X\} \subset V_{x, 0}$ of an $\mathfrak{s l}_{2}(\mathfrak{f})$-triple in $\overline{\mathfrak{g}}_{x}$ completing $e$ such that $F_{L}^{*}$ is not maximal in $\mathcal{B}_{L}(Y, H, X)$. Then, there exists some generalized $L$-facet $C_{L}^{*}$ in $\mathcal{B}_{L}(Y, H, X)$ containing $F_{L}^{*}$ in its closure. In particular, by [[12], Corollary 3.2.19], we may identify $V_{C_{L}^{*}}$ with a Levi subalgebra of the parabolic subalgebra $\mathfrak{g}_{C_{L}^{*}} / \mathfrak{g}_{F_{L}^{*}}^{+}$, which are both $L$ stable by the proof of Proposition III.9. Let $\mathcal{A}$ be an apartment in $\mathcal{B}(H)$ containing $x$, and let $\mathbf{S}$ be the maximal $k$-split torus in $\mathbf{H}$ corresponding to $\mathcal{A}$. By [[17], Proposition $5.4]$ and [[26], Theorem 1.9], we can choose some $L$-stable maximal $k$-split torus $\mathbf{S}^{\prime}$ containing $\mathbf{S}$ such that $\mathcal{A}(\mathbf{S}, k) \subset \mathcal{A}\left(\mathbf{S}^{\prime}, k\right)$. Let $\mathbf{S}$ denote the maximal $\mathfrak{f}$-split torus in $\mathrm{H}_{x}$ corresponding to $\mathbf{S}$. In the notation of [[15], Lemma 3.3] (letting $k=\mathfrak{f}$ and $\lambda=\mu)$, there exists some $\mu \in \mathbf{X}_{*}^{\mathfrak{f}}(\mathrm{S})$ such that $V_{C_{L}^{*}}=C_{V_{F_{L}^{*}}}(d \mu)$. In particular, since

$C_{L}^{*} \subset \mathcal{B}_{L}(Y, H, X)$, we have $e \in V_{C_{L}^{*}}$. Thus, there is a semisimple element in $V_{F_{L}^{*}}^{L}$ which centralizes $e$ and lies in the Lie algebra of some $\mathrm{f}$-split torus in $\mathrm{H}_{x}$. Moreover, 
this element does not lie in the center of $V_{F_{L}^{*}}$ since $V_{C_{L}^{*}}$ is properly contained in $V_{F_{L}^{*}}$.

$(2) \Rightarrow(1)$ : We prove the contrapositive. Let $x \in F_{L}^{*}$. Suppose there is some element $s \in C_{V_{F_{L}^{*}}^{L}}(e) \cap\left[V_{F_{L}^{*}}, V_{F_{L}^{*}}\right]$, which lies in the Lie algebra of some $\mathfrak{f}$-split torus in $\mathrm{H}_{x}$. By $[[3], 8.15(\mathrm{~d})]$, we may assume there is some one-parameter subgroup $\bar{\mu} \in \mathbf{X}_{*}^{\mathfrak{f}}\left(\mathbf{H}_{x}\right)$ for which $s \in \operatorname{im}(d \bar{\mu})$. By [[17], Proposition 3.4.1] or [[15], Proposition 2.3], there is some $L$-stable maximal $\mathfrak{f}$-split torus $T$ in $\mathrm{G}_{x}$ such that $\bar{\mu} \in \mathbf{X}_{*}^{\mathfrak{f}}(\mathbf{T})$. Since $\bar{\mu}$ is $L$-fixed, $\bar{\mu}$ determines a $L$-stable $\mathfrak{f}$-parabolic subgroup $\mathrm{P}$ of $\mathrm{G}_{x}$ which contains a $L$-stable $\mathfrak{f}$-Levi subgroup $\mathrm{M}:=C_{\mathrm{G}_{x}}(\bar{\mu})$. Let $\mathfrak{m}$ denote the Lie algebra of $\mathrm{M}$, which lies inside the Lie algebra of P. By [[17], Proposition 5.4], we can choose a $L$-stable maximal $k$-split torus $\mathbf{T}$ such that $\mathcal{A}(\mathbf{T}, k)$ contains $x$ and $\mathcal{A}(\mathbf{T}, k)^{L}$ is an affine space whose dimension is equal to the dimension of a maximal $k$-split torus of $\mathbf{H}$. Using the embedding from [[26], Theorem 1.9], we must have that $\mathcal{A}(\mathbf{T}, k)^{L}$ is an apartment of $\mathcal{B}(H)$ containing $x$. The image of $\mathbf{T}$ in $\mathbf{G}_{x}$ is maximal $\mathfrak{f}$-split torus $\mathbf{S}$ for which $\mathbf{S}^{L}$ is a maximal $\mathfrak{f}$-split torus in $\mathrm{H}_{x}$. Thus, by conjugacy of maximal $\mathfrak{f}$-split tori in $\mathrm{H}_{x}$, we may assume that the image of $\mathbf{T}$ in $\mathrm{G}_{x}$ is $\mathbf{T}$. Using the identification of $\mathbf{X}_{*}^{k}(\mathbf{T})$ with $\mathbf{X}_{*}^{\mathfrak{f}}(\mathbf{T})$, let $\mu$ be a lift of $\bar{\mu}$ in $\mathbf{X}_{*}^{k}(\mathbf{T}) \otimes \mathbb{R}$, let $C_{L}^{*}$ be the first $L$-facet of $\mathcal{A}(\mathbf{T}, k)$ encountered when moving from $x$ to $x+\mu$. Then, the vector space of $\mathfrak{f}$-rational points of the Lie algebra of $\mathrm{P}$ is of the form $\mathfrak{g}_{C_{L}^{*}} / \mathfrak{g}_{F_{L}^{*}}^{+}$. By our choice of $\bar{\mu}$, we have $e \in \mathfrak{m} \subset \mathfrak{g}_{C_{L}^{*}} / \mathfrak{g}_{F_{L}^{*}}$.

Let $e=X^{\prime}+\mathfrak{g}_{F_{L}^{*}}^{+}$, for some representative $X^{\prime}$ which lies in $\mathfrak{g}_{L}^{*}$. If $e$ is trivial, then we complete $e$ to the trivial $\mathfrak{s l}_{2}(\mathfrak{f})$-triple inside $V_{C_{L}^{*}}$ and let $\{Y, H, X\}$ be the trivial $\mathfrak{s l}_{2}(k)$-triple in $\mathfrak{g}_{L}^{*}$ lifting $\{f, h, e\}$. This shows that $F_{L}^{*}$ cannot be maximal in $\mathcal{B}_{L}(Y, H, X)$.

Suppose $e$ is nontrivial. We want to show that there exists some lift $X$ of $e$ in $\mathfrak{g}_{C_{L}^{*}} \cap \mathcal{N}$. Since $e$ is degenerate in $V_{F_{L}^{*}}$, using the same argument as that given in the last paragraph of Lemma IV.2, there exists some $X \in \mathfrak{g}_{C_{L}^{*}} \cap \mathcal{N}$ whose image in $\mathfrak{g}_{C_{L}^{*}} / \mathfrak{g}_{F_{L}^{*}}^{+}$is $e$. Since $e$ is a nonzero element of $\mathfrak{m}$, it does not lie in the kernel $\mathfrak{g}_{C_{L}^{*}}^{+} / \mathfrak{g}_{F_{L}^{*}}^{+}$ 
of the projection from $\mathfrak{g}_{C_{L}^{*}} / \mathfrak{g}_{F_{L}^{*}}^{+}$onto $\mathfrak{m}$. In particular, we have that $X \notin \mathfrak{g}_{C_{L}^{*}}^{+}$. Thus, by Hypothesis IV.3, there exists some $\mathfrak{s l}_{2}(\mathfrak{f})$-triple $\{f, h, e\}$ in $V_{C_{L}^{*}}$ completing $e$, and by Corollary IV.13, there exists a lift $\{Y, H, X\}$ of $\{f, h, e\}$ which lies in $\mathfrak{g}_{C_{L}^{*}}$. This shows that $F_{L}^{*}$ cannot be maximal in $\mathcal{B}_{L}(Y, H, X)$.

Lemma V.21. Suppose all hypotheses in Section IV.1 hold. If $\left(F_{L}^{*}, e\right) \in I_{r}^{n}$ and e is nontrivial, then $\left(F_{L}^{*}, e\right) \in I_{r}^{d}$ if and only if there exists some $x \in F_{L}^{*}$, a normal $\mathfrak{s l}_{2}(\mathfrak{f})$ triple $(f, h, e) \in V_{x,-r}^{-1} \times V_{x, 0}^{0} \times V_{x, r}^{1}$ completing e, and a normal $\mathfrak{s l}_{2}(k)$-triple $\{Y, H, X\}$ in $\mathfrak{g}$ lifting $\{f, h, e\}$ such that $F_{L}^{*}$ is a maximal generalized $(r, L)$-facet of $\mathcal{B}_{L}(Y, H, X)$.

Proof. " $\Rightarrow: "$ This is a consequence of the definition.

" $\Leftarrow:$ " Let $x \in F_{L}^{*},\{f, h, e\}$, and $\{Y, H, X\}$ be data satisfying the hypotheses of the lemma such that $F_{L}^{*}$ is a maximal generalized $(r, L)$-facet of $\mathcal{B}_{L}(Y, H, X)$. Suppose there is some $x^{\prime} \in F_{L}^{*}$, a normal $\mathfrak{s l}_{2}(\mathfrak{f})$-triple $\left(f^{\prime}, h^{\prime}, e\right) \in V_{x^{\prime},-r}^{-1} \times V_{x^{\prime}, 0}^{0} \times V_{x^{\prime}, r}^{1}$ completing $e$, and a normal $\mathfrak{s l}_{2}(k)$-triple $\left\{Y^{\prime}, H^{\prime}, X^{\prime}\right\}$ in $\mathfrak{g}$ lifting $\left\{f^{\prime}, h^{\prime}, e\right\}$. We argue by contradiction, and suppose $F_{L}^{*}$ is not maximal in $\mathcal{B}_{L}\left(Y^{\prime}, H^{\prime}, X^{\prime}\right)$. Since $x, x^{\prime} \in F_{L}^{*}$, we have $\mathfrak{g}_{x^{\prime},-r}=\mathfrak{g}_{x,-r}$ and $\mathfrak{g}_{x, r}=\mathfrak{g}_{x^{\prime}, r}$. Since $\left[\mathfrak{g}_{x^{\prime},-r}, \mathfrak{g}_{x^{\prime}, r}\right] \subset \mathfrak{g}_{x, 0}$, the lift $\left(Y^{\prime}, H^{\prime}, X^{\prime}\right)$ lies in $\mathfrak{g}_{x,-r}^{-1} \times \mathfrak{h}_{x, 0} \times \mathfrak{g}_{x, r}^{1}$, so we may assume $x^{\prime}=x$. By Lemma V.14, we have that ${ }^{H} X=\mathcal{O}_{1}\left(F_{L}^{*}, e\right)={ }^{H} X^{\prime}$. Combining this with Corollary V.9, $X$ is $H_{x}^{+}$-conjugate to $X^{\prime}$. Thus, by replacing $\left\{Y^{\prime}, H^{\prime}, X^{\prime}\right\}$ with some $H_{x}^{+}$-conjugate, we may assume $X=X^{\prime}$. By the last line of Hypothesis IV.9, there exists some element $h \in C_{H}(X)$ with ${ }^{h} Y=Y^{\prime}$ and ${ }^{h} H=H^{\prime}$. In particular, $Y \in{ }^{h^{-1}} \mathfrak{g}_{x,-r}=\mathfrak{g}_{h^{-1} x,-r}$ and $X \in$ ${ }^{h^{-1}} \mathfrak{g}_{x, r}=\mathfrak{g}_{h^{-1} x, r}$, so $h^{-1} \mathcal{B}_{L}\left(Y^{\prime}, H^{\prime}, X\right)=\mathcal{B}_{L}(Y, H, X)$. This shows that $\mathcal{B}_{L}\left(Y^{\prime}, H^{\prime}, X\right)$ and $\mathcal{B}_{L}(Y, H, X)$ have the same dimension. However, we assumed that $F_{L}^{*}$ was not maximal in $\mathcal{B}_{L}\left(Y^{\prime}, H^{\prime}, X\right)$, so $h^{-1} F_{L}^{*}$ is not maximal in $\mathcal{B}_{L}(Y, H, X)$, which is a contradiction since the action of $H$ preserves dimension. 
Remark V.22. Suppose $\left(F_{1, L}^{*}, e_{1}\right) \sim\left(F_{2, L}^{*}, e_{2}\right)$. As a consequence of the proof of Lemma V.21, we have $\left(F_{1, L}^{*}, e_{1}\right) \in I_{r}^{d}$ if and only if $\left(F_{2, L}^{*}, e_{2}\right) \in I_{r}^{d}$.

Theorem V.23. Suppose all hypotheses of Section IV.1 hold. There is a bijective correspondence between $I_{r}^{d} / \sim$ and $\mathcal{O}_{1}(0)$ given by the map that sends $\left(F_{L}^{*}, e\right)$ to $\mathcal{O}_{1}\left(F_{L}^{*}, e\right)$.

Proof. We have already shown that the map which sends $\left(F_{L}^{*}, e\right)$ to $\mathcal{O}_{1}\left(F_{L}^{*}, e\right)$ is welldefined in Lemma V.17. We will first show that the map restricted to $I_{r}^{d}$ is injective. Suppose $\mathcal{O}_{1}\left(F_{1, L}^{*}, e_{1}\right)=\mathcal{O}_{1}\left(F_{2, L}^{*}, e_{2}\right)$. Recall from Corollary III.22 that for $F_{L}^{*} \in \mathcal{F}(r)$, the set $C\left(F_{L}^{*}\right)$ is nonempty. Complete $e_{i}$ to an $\mathfrak{s l}_{2}(\mathfrak{f})$-triple $\left(f_{i}, h_{i}, e_{i}\right)$ in $V_{F_{i, L}^{*}}^{-1} \times V_{F_{i, L}^{*}}^{0} \times$ $V_{F_{i, L}^{*}}^{1}$. By Corollary IV.13, we can lift these $\mathfrak{s l}_{2}(\mathfrak{f})$-triples to $\mathfrak{s l}_{2}(k)$-triples $\left\{Y_{i}, H_{i}, X_{i}\right\}$ in $\mathfrak{g}$. By Lemma V.14, $\mathcal{O}_{1}\left(F_{i, L}^{*}, e_{i}\right)={ }^{H} X_{i}$. Since $\mathcal{O}_{1}\left(F_{1, L}^{*}, e_{1}\right)=\mathcal{O}_{1}\left(F_{2, L}^{*}, e_{2}\right)$, we thus have ${ }^{H} X_{1}={ }^{H} X_{2}$, so by Hypothesis IV.9, there exists an $h \in H$ such that $\left\{Y_{1}, H_{1}, X_{1}\right\}=\left\{{ }^{h} Y_{2},{ }^{h} H_{2},{ }^{h} X_{2}\right\}$. By Lemma V.14, we have $F_{1, L}^{*} \subset \mathcal{B}_{L}\left(Y_{1}, H_{1}, X_{1}\right)$, and $h F_{2, L}^{*} \subset \mathcal{B}_{L}\left(Y_{1}, H_{1}, X_{1}\right)$, so since $\left(F_{i, L}^{*}, e_{i}\right) \in I_{r}^{d}, F_{1, L}^{*}$ and $h F_{2, L}^{*}$ are maximal generalized $(r, L)$-facets of $\mathcal{B}_{L}\left(Y_{1}, H_{1}, X_{1}\right)$. Thus, by Lemma V.6, $F_{1, L}^{*}$ and $h F_{2, L}^{*}$ are strongly $r$-associated. In particular, there exists an apartment $\mathcal{A} \subset \mathcal{B}(H)$ such that

$$
A\left(\mathcal{A}, F_{1, L}^{*}\right)=A\left(\mathcal{A}, h F_{2, L}^{*}\right) \neq \emptyset .
$$

As $X_{1}$ has image $e_{1}$ in $V_{F_{1, L}^{*}}$ and $X_{1}$ has image ${ }^{h} e_{2}$ in $V_{h F_{2, L}^{*}}$, the element $X_{1}$ lies in $\mathfrak{g}_{F_{1, L}^{*}}^{1} \cap \mathfrak{g}_{h F_{2, L}^{*}}^{1}$, and

$$
e_{1}={ }^{h} e_{2} \text { in } V_{F_{1, L}^{*}}^{1}=V_{h F_{2, L}^{*}}^{1}
$$

Thus, in particular, $\left(F_{1, L}^{*}, e_{1}\right) \sim\left(F_{2, L}^{*}, e_{2}\right)$, i.e the map is injective.

For surjectivity, first let $\{0\}$ be the trivial orbit. Let $F_{L}^{*}$ be an open generalized $(r, L)$-facet, and let $e$ be the trivial element of $V_{F_{L}^{*}}^{1}$. Then, $\left(F_{L}^{*}, e\right)$ maps to $\{0\}$. Now, suppose $\mathcal{O}$ is nontrivial and let $X \in \mathcal{O}$. Complete $X$ to an $\mathfrak{s l}_{2}(k)$-triple $\{Y, H, X\}$ and 
choose a maximal generalized $(r, L)$-facet $F_{L}^{*} \subset \mathcal{B}_{L}(Y, H, X)$. Let $e$ denote the image of $X$ in $V_{F_{L}^{*}}$. Then by Lemma V.14 (2), we have $\mathcal{O}_{1}\left(F_{L}^{*}, e\right)={ }^{H} X$. 
APPENDIX 


\section{APPENDIX A}

\section{Compatibilities and an example}

In [[14], Lemma 2.14], Hakim and Murnaghan show that any subgroup $\mathcal{G}$ of the Moy-Prasad filtration subgroups $P^{(i)}$ corresponding to positive depths are strongly 2-divisible under a tameness assumption. Their result is then used to show that first (non-abelian) cohomology group $H^{1}(\langle\theta\rangle, \mathcal{G})$ is trivial. In this section, we let $L$ be a finite group acting on $\mathbf{G}$ by $k$-automorphisms and show that $H^{1}\left(L, P^{(1)}\right)$ is trivial if $p$ does not divide the order of $L$. This result is not new, but we provide a proof for completeness.

Proposition A.1. The first cohomology group $H^{1}\left(L, P^{(1)}\right)$ is trivial.

Proof. By [[25], 2.16], the quotient $P^{(i)} / P^{(i+1)}$ has the structure of a finite-dimensional $\mathfrak{f}$-vector space. Moreover, since $P^{(1)}$ is a pro- $p$ group, we have

$$
P^{(1)}=\lim _{\longleftarrow} P^{(i)} / P^{(i+1)} .
$$

Since $L$ is a finite group whose order is coprime to $p$, by [[29], Corollary 7.8], we have

$$
H^{1}\left(L, P^{(i)} / P^{(i+1)}\right)=1,
$$

so $H^{1}\left(L, P^{(1)}\right)$ is trivial. 
The following corollary is immediate from the proposition:

Corollary A.2. Let $\theta$ be a k-automorphism of $\boldsymbol{G}$ of order $m \geq 2$. Then, if $p$ does not divide $m$ and $i \geq 1, H^{1}\left(\langle\theta\rangle, P^{(1)}\right)$ is trivial.

Note that $\mathbf{G}^{L} /\left(\mathbf{G}^{L}\right)^{\circ}$ is a finite group.

Lemma A.3. Let $x \in \mathcal{B}(H)$. Then, if $\left(G^{L}\right)^{\circ}$ is the group of $k$-rational points of $\left(\boldsymbol{G}^{L}\right)^{\circ}$, we have

$$
G_{x}^{+} \cap H=G_{x}^{+} \cap\left(G^{L}\right)^{\circ}
$$

Proof. Let $h \in G_{x}^{+} \cap H$. Since every element in $\mathbf{G}^{L} /\left(\mathbf{G}^{L}\right)^{\circ}$ has finite order, we have $h^{n} \in G_{x}^{+} \cap\left(G^{L}\right)^{\circ}$, for some $n \in \mathbb{N}$. By the proof of Corollary A.2 and the proof of [[14], Proposition 2.12], we have that the group $G_{x}^{+} \cap\left(G^{L}\right)^{\circ}$ is strongly $n$-divisible (since $p$ is coprime to $|L|$ ). In particular, since $h^{n}$ has a unique $n^{\text {th }}$ root $s$ in $G_{x}^{+} \cap H$, which lies in $G_{x}^{+} \cap\left(G^{L}\right)^{\circ}$, we must have $h \in G_{x}^{+} \cap\left(G^{L}\right)^{\circ}$.

We claim that when $x \in \mathcal{B}(H)$, we have

$$
G_{x}^{+} \cap H=H_{x}^{+}
$$

We first prove this statement when $\mathbf{H}$ is connected, and then apply Lemma A.3.

Proposition A.4. Suppose $\boldsymbol{H}=\left(\boldsymbol{G}^{L}\right)^{\circ}$, and let $x \in \mathcal{B}(H)$. Then,

$$
G_{x}^{+} \cap H=H_{x}^{+}
$$

Proof. If $p=\infty$, every maximal $k$-torus splits over a tamely ramified extension, so, in particular, $H_{x}^{+}=G_{x}^{+} \cap H$ by [[2], 3.6]. For $p$ finite, we note that if $h \in H_{x}^{+}$, it is topologically $k$-unipotent (in the sense of [[30], Definition 2.15]), so by [[30], Lemma 
2.21], we have $\lim _{n \rightarrow \infty} h^{p^{n}}=1$. Thus, $h$ is topologically $k$-unipotent in $G$, so $h$ lies in $\mathbf{G}(E)_{0^{+}}$, for some finite extension $E / k$. Combining [[12], 4.5.1] with [[1], Proposition 1.4.1], we have that $h \in G_{x}^{+}$, so $H_{x}^{+} \subset G_{x}^{+} \cap H$.

Let $h \in G_{x}^{+} \cap H$. When $p$ is finite, we know that $h$ is topologically $k$-unipotent in $G$, and thus in $H$, so $h \in \mathbf{H}(E)_{0^{+}}$, for some finite extension $E / k$. Since $h$ also lies in $\operatorname{stab}_{H}(x) \subset \operatorname{stab}_{\mathbf{H}(E)}(x)$, by [[12], Lemma 4.5.1], we have $h \in \mathbf{H}(E)_{x}^{+}$. By [[1], Proposition 1.4.1], we have $h \in H_{x}^{+}$.

Combining Proposition A.4 and Lemma A.3, the following corollary is immediate.

Corollary A.5. For $\boldsymbol{H}$ as in Chapter II, and $x \in \mathcal{B}(H)$, we have

$$
G_{x}^{+} \cap H=H_{x}^{+}
$$

To each facet $F^{\prime} \subset \mathcal{B}(G)$, one associates a connected, reductive f-group which we have denoted $\mathrm{G}_{F^{\prime}}$. This group may be thought of as the maximal reductive quotient of the special fiber of the Bruhat-Tits group scheme associated to some point $x \in F^{\prime}$ or as the quotient $\mathbf{G}(K)_{x} / \mathbf{G}(K)_{x}^{+}$. In order to prove that $\left(\mathrm{G}_{F}^{L}\right)^{\circ}=\mathrm{H}_{F}$, for $F^{\prime}$ an $L$-stable facet in $\mathcal{B}(G)$, it is more convenient to choose the latter description. In the statement of the proposition below, we let $\left(\mathrm{G}_{x}^{L}\right)^{\circ}$ denote the Zariski-connected component of the $\mathfrak{F}$-group $\mathrm{G}_{x}^{L}$, for $x \in \mathcal{B}(H)$.

Proposition A.6. Let $x \in \mathcal{B}(H)$. Then,

$$
\left(G_{x}^{L}\right)^{\circ}=H_{x}
$$

Proof. Let $\hat{K}$ denote the completion of $K$ in $\bar{k}$. Then, since the residue fields of $K$ and $\hat{K}$ coincide, it suffices to prove this statement over $\hat{K}$. By the proof of $[[26]$, Prop. 1.7], we have $\mathbf{H}(\hat{K})_{x} \subset \mathbf{G}(\hat{K})_{x}$. By Corollary A.5, the map $\mathbf{H}(\hat{K})_{x} / \mathbf{H}(\hat{K})_{x}^{+} \rightarrow$ 
$\mathbf{G}(\hat{K})_{x} / \mathbf{G}(\hat{K})_{x}^{+}$induced by the map $\mathbf{H}(\hat{K})_{x} \rightarrow \mathbf{G}(\hat{K})_{x}$ is an injection. Moreover, since $\mathrm{H}_{x}$ is connected, we must have $\mathrm{H}_{x} \subset\left(\mathrm{G}_{x}^{L}\right)^{\circ}$. Applying Lemma III.26 (with $r=0$ ), the Lie algebras of $\mathrm{H}_{x}$ and $\left(\mathrm{G}_{x}^{L}\right)^{\circ}$ coincide, so the result follows.

For the remainder of this appendix, we enumerate the set of nilpotent orbits associated to the pair $\left(\mathbf{S L}_{3}, \mathbf{P G L}_{2}\right)$.

Recall that we have an involution $\theta: \mathbf{S L}_{3} \rightarrow \mathbf{S L}_{3}$ defined by $A \mapsto J\left(A^{t}\right)^{-1} J$, where $J=\left(\begin{array}{lll}0 & 0 & 1 \\ 0 & 1 & 0 \\ 1 & 0 & 0\end{array}\right)$. The Lie algebra of $\mathbf{S L}_{3}$, denoted $\mathfrak{s l}_{3}$, has a decomposition on the level of $k$-points which is given by

$$
\mathfrak{s l}_{3}(k)=\left(\begin{array}{ccc}
a & b & 0 \\
c & 0 & -b \\
0 & -c & -a
\end{array}\right) \oplus\left(\begin{array}{ccc}
x & y & s \\
z & -2 x & y \\
u & z & x
\end{array}\right),
$$

with $a, b, c, s, u, x, y, z \in k$. Let $X=\left(\begin{array}{ccc}x & y & s \\ z & -2 x & y \\ u & z & x\end{array}\right)$ be nilpotent. We would like to find a nicer representative for $X$ up to $H=\mathbf{P G L}_{2}(k)$-conjugacy.

By Remark IV.5, since $X$ is nilpotent, there is a one-parameter subgroup $\lambda$ : $\mathbf{G L}_{1} \rightarrow \mathbf{H}$ such that ${ }^{\lambda(t)} X=t^{2} X$. On the other hand, since the image of $\lambda$ lies in a maximal $k$-split torus of $\mathbf{H}$, there is some $h \in \mathbf{H}$ and $n \in \mathbb{Z}$ such that $\left({ }^{h} \lambda\right)(t)=$ $\left(\begin{array}{ccc}t^{n} & 0 & 0 \\ 0 & 1 & 0 \\ 0 & 0 & t^{-n}\end{array}\right)$. Thus, we have ${ }^{\left({ }^{h} \lambda\right)(t)}\left({ }^{h} X\right)=t^{2}\left({ }^{h} X\right)$. Letting $Z={ }^{h} X=\left(\begin{array}{ccc}u & v & w \\ r & -2 u & v \\ s & r & u\end{array}\right)$, we have

$$
\left(\begin{array}{ccc}
u & t^{n} v & t^{2 n} w \\
t^{-n} r & -2 u & t^{n} v \\
t^{-2 n} s & t^{-n} r & u
\end{array}\right)=\left(\begin{array}{ccc}
t^{2} u & t^{2} v & t^{2} w \\
t^{2} r & -2 u t^{2} & t^{2} v \\
t^{2} s & t^{2} r & t^{2} u
\end{array}\right) .
$$


Assume $X$ is nontrivial. If $v \neq 0$, we must have $n=2$, but this forces all other entries to be zero. If $w \neq 0$, then, $n$ must be equal to 1 , but this forces all other entries to be zero. Thus, the nilpotent $H$-conjugacy classes in the $(-1)$-eigenspace of $\mathfrak{s l}_{3}(k)$ under $d \theta$ lie in one of the three subsets of $\mathfrak{s l}_{3}(k)$ :

$$
\{\operatorname{triv}\},\left\{\left(\begin{array}{ccc}
0 & v & 0 \\
0 & 0 & v \\
0 & 0 & 0
\end{array}\right) \mid v \in \mathbb{Q}_{p}^{\times}\right\} \text {, and }\left\{\left(\begin{array}{ccc}
0 & 0 & w \\
0 & 0 & 0 \\
0 & 0 & 0
\end{array}\right) \mid w \in \mathbb{Q}_{p}^{\times}\right\} \text {, }
$$

where triv denotes the trivial orbit.

We note that all matrices of the form $\left(\begin{array}{ccc}0 & v & 0 \\ 0 & 0 & v \\ 0 & 0 & 0\end{array}\right)$ are $\mathbf{H}(k)$-conjugate by the diagonal maximal $\mathbb{Q}_{p}$-split torus. Matrices of the form $\left(\begin{array}{ccc}0 & 0 & w \\ 0 & 0 & 0 \\ 0 & 0 & 0\end{array}\right)$ split up into four conjugacy classes which are parametrized by $\left(\mathbb{Q}_{p}\right)^{\times} /\left(\mathbb{Q}_{p}^{\times}\right)^{2}$. 


\section{BIBLIOGRAPHY}




\section{BIBLIOGRAPHY}

[1] J.D. Adler. Refined anisotropic $K$-types and supercuspidal representations. $P a$ cific J. Math., 185(1):1-32, 1998.

[2] J.D. Adler and Stephen DeBacker. Some applications of Bruhat-Tits theory to harmonic analysis on the Lie algebra of a reductive $p$-adic group. Michigan Math J., 50(2):263-286, 2002.

[3] A Borel. Linear algebraic groups. Springer, New York, 1991.

[4] Francois Bruhat and Jacques Tits. Groupes réductifs sur un corps local,. Inst. Hautes Études Sci. Publ. Math., (41):5-251, 1972.

[5] Francois Bruhat and Jacques Tits. Groupes réductifs sur un corps local. ii. Schémas en groupes. Existence d'une donnée radicielle valuée. Inst. Hautes Études Sci. Publ. Math., (60):197-376, 1984.

[6] R. W. Carter. Finite Groups of Lie type. Wiley, Chichester, 1993.

[7] D.H. Collingwood and W.M McGovern. Nilpotent Orbits in semisimple Lie algebras. Van Nostrand Reinhold, New York, 1993.

[8] Brian Conrad, Ofer Gabber, and Gopal Prasad. Pseudo-reductive groups. Cambridge University Press, Cambridge, 2010.

[9] S DeBacker. Homogeneity results for invariant distributions of a reductive $p$-adic group. Ann. Sci. École Norm. Sup. (4), 35(3):391-422, 2002.

[10] S DeBacker. Parametrizing conjugacy classes of maximal unramified tori via Bruhat-Tits theory. Michigan Math J., 54(1):157-178, 2006.

[11] S. DeBacker and Mark Reeder. Depth-zero supercuspidal $L$-packets and their stability. Ann. of Math (2), 169(3):795-901, 2009.

[12] Stephen DeBacker. Parametrizing nilpotent orbits via Bruhat-Tits theory. Ann. Of Math (2), 156(1):295-332, 2002.

[13] B. Gross, M. Reeder, and J.K. Yu. Invariant theory and unrefined minimal $K$-types. work in progress.

[14] J. Hakim and F. Murnaghan. Distinguished tame supercuspidal representations. Int. Math. Res. Pap. IMRP, Art. ID rpn005(2):166pp., 2008. 
[15] A. G. Helminck and S. P. Wang. On rationality properties of involutions of reductive groups. Adv. Math, 99(1):26-96, 1993.

[16] G. R. Kempf. Instability in invariant theory. Ann. of Math. (2), 108(2):299-316, 1978.

[17] J.-L. Kim and A. Moy. Involutions, classical groups, and buildings. J. Algebra, 242(2):495-515, 2001.

[18] B. Kostant. The principal three-dimensional subgroup and the Betti numbers of a complex simple lie group. Amer. J. Math., 81:973-1032, 1959.

[19] B. Kostant and S. Rallis. Orbits and representations associated with symmetric spaces. Amer. J. Math., 93:753-809, 1971.

[20] P. Levy. Vinberg's $\theta$-groups in positive characteristic and Kostant-Weierstrass slices. Transform. Groups, 14(2):417-461, 2009.

[21] A. Moy and Gopal Prasad. Unrefined minimal $K$-types for $p$-adic groups. Invent. Math., 116(1-3):393-408, 1994.

[22] A. G Noël. Nilpotent orbits and theta-stable parabolic subalgebras. Represent. Theory, 2:1-32, 1998.

[23] D.I. Panyushev. On invariant theory of $\theta$-groups. J. Algebra, 283(2):655-670, 2005.

[24] V.L. Popov. Representations with a free module of covariants. Funkcional. Anal. i Priložen, 10(3):91-92, 1976.

[25] Gopal Prasad and M. S. Raghunathan. Topological central extensions of semisimple groups over local fields. Ann. of Math. (2), 119(1):143-201, 1984.

[26] Gopal Prasad and J.-K. Yu. On finite group actions on reductive groups and buildings. Invent. Math., 147(3):545-560, 2002.

[27] C. Rader and S. Rallis. Spherical characters on $\mathfrak{p}$-adic symmetric spaces. Amer. J. Math., 118(1):91-178, 1996.

[28] R.W. Richardson. Orbits, invariants, and representations associated to involutions of reductive groups. Invent. Math., 66(2):287-312, 1982.

[29] Romyar Sharifi. An introduction to group cohomology, notes found at www . math . arizona.edu/ sharifi/groupcoh.pdf. 2010.

[30] L. Spice. Topological Jordan decompositions. J. Algebra, 319(8):3141-3163, 2008.

[31] Jacques Tits. Reductive groups over local fields. Automorphic forms, representations and L-functions (Proc. Sympos. Pure Math., Oregon State Univ., Corvallis, Ore., 1977), Part 1, 1977. 
[32] E.B. Vinberg. The Weyl group of a graded Lie algebra. Izv. Akad. Nauk SSSR Ser. Mat., 1976.

[33] T. Vust. Opération de groupes réductifs dans un type de cones presque homogènes. Bull. Soc. Math. France, 102:317-333, 1974.

[34] J.-L. Waldspurger. Intégrales orbitales nilpotentes et endoscopie pour les groupes classiques non ramifiés. Astérisque, vi(269):449, 2001.

[35] J.-K. Yu. Construction of tame supercuspidal representations. J. Amer. Math. Soc., 50(2):263-286, 2002.

[36] J.K. Yu. Smooth models associated to concave functions in Bruhat-Tits theory. preprint, 2002. 